\title{
GaN:Gd - \\ Ein verdünnter magnetischer Halbleiter?
}

\author{
Dissertation \\ zur Erlangung des Doktorgrades \\ der Mathematisch-Naturwissenschaftlichen Fakultäten \\ der Georg-August-Universität Göttingen
}

\author{
Vorgelegt von \\ Martin Röver \\ aus Leer (Ostfriesland).
}

Göttingen, 2010 
D7

Referentin: Prof. Angela Rizzi

Korreferent: Prof. Axel Hoffmann

Tag der mündlichen Prüfung: 


"Die Physik ist im moment wieder sehr verfahren, für mich ist sie jedenfalls viel zu schwierig und ich wollte, ich wäre Filmkomiker oder so etwas und hätte nie etwas von Physik gehört."

Pauli an Kronig, 21.5.1925, PBW 



\section{Inhaltsverzeichnis}

$\begin{array}{ll}\text { Einleitung } & 1\end{array}$

1 GaN:Gd - Ein Verdünnter Magnetischer Halbleiter? $\mathbf{5}$

1.1 Strukturelle, magnetische und elektrische Eigenschaften . . . . . . . . 6

1.2 Kolossale Momente und deren Herkunft . . . . . . . . . . . . . . . . 8

1.2.1 Phänomenologisches Modell von Dhar et al. . . . . . . . . . . . 9

1.2.2 Kolossale Momente durch Defekte . . . . . . . . . . . . . . . 10

1.3 Theoretische Modelle . . . . . . . . . . . . . . . . . . . . . . . . 11

1.4 Ladungsträger-induzierter Magnetismus . . . . . . . . . . . . . . . . 12

1.5 Defekte und Dotierungen in GaN . . . . . . . . . . . . . . . 15

1.6 Die Rolle der Galliumleerstelle . . . . . . . . . . . . . . . . . . . . 18

1.7 Intrinsische Defekte und Sauerstoff . . . . . . . . . . . . . . . . . . . . . . . . . . . . . . .

Zusammenfassung . . . . . . . . . . . . . . . . . . . 21

2 Herstellung von Gadolinium dotiertem GaN 23

2.1 ELSA - Epitaxie LEED und Spektroskopie Anlage . . . . . . . . . . . . . . . . 24

2.2 Substratwahl und -temperatur . . . . . . . . . . . . . . . . . . . . . . . . . 25

2.3 Vorbehandlung . . . . . . . . . . . . . . . . . . . 27

2.4 Herstellung von GaN:Gd mit MBE . . . . . . . . . . . . . . . 27

2.5 Einfluß der Gd-Dotierung auf die Wachstumsrate . . . . . . . . . . . . . . . . 29

Zusammmenfassung . . . . . . . . . . . . . . . . . 29

3 Gadolinium Konzentration $\quad 31$

3.1 SIMS Standard und TRIM Simulation . . . . . . . . . . . . . . . . . 32

3.2 Masseninterferenzen . . . . . . . . . . . . . . . . . . . 33

3.3 Aufösungsvermögen und Fehler . . . . . . . . . . . . . . . . . . . . . . . . . . . . . . . . 34

3.4 Auswertung des Standards . . . . . . . . . . . . . . . . . . . . . . . . . . . . . . . . 34

3.5 Auswertung der Proben . . . . . . . . . . . . . . . . . 35

3.6 Ergebnisse in den beiden Serien . . . . . . . . . . . . . . . 36

3.7 Theoretische Abschätzung der Gd Konzentration . . . . . . . . . . . . . . . 38

3.8 Verunreinigungen . . . . . . . . . . . . . . . . . . . . . . . . . 39

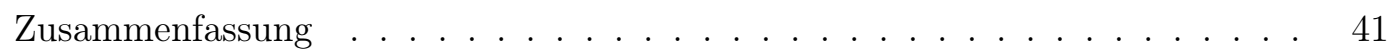


4 Magnetische Eigenschaften 43

4.1 SQUID Messungen . . . . . . . . . . . . . . . . . . . . 44

4.1.1 Systematische Fehler bei SQUID-Messungen . . . . . . . . . . . . 44

4.1 .2 Meßsprozedur . . . . . . . . . . . . . . . . . 46

4.1.3 Korrekturen der Rohdaten . . . . . . . . . . . . . . . . . . . . 46

4.2 Übersicht und Reproduzierbarkeit . . . . . . . . . . . . . . . . . . . . 47

4.3 Nicht co-dotiertes GaN:Gd . . . . . . . . . . . . . . . . . . . 48

4.4 Co-Dotierungen . . . . . . . . . . . . . . . . . . 50

4.4 .1 Silizium-co-Dotierung . . . . . . . . . . . . . . . 50

4.4 .2 Wasserstoff-co-Dotierung . . . . . . . . . . . . . . . . 51

4.4 .3 Sauerstoff-co-Dotierung . . . . . . . . . . . . . . . 51

4.5 Allgemeine Trends . . . . . . . . . . . . . . . . . . . . . . . . 52

4.6 Effektive Magnetisierung . . . . . . . . . . . . . . . . . . . . . . . . . . 54

4.7 Ausscheidungen . . . . . . . . . . . . . . . . . . . . . 55

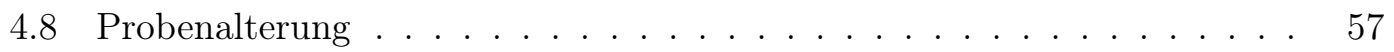

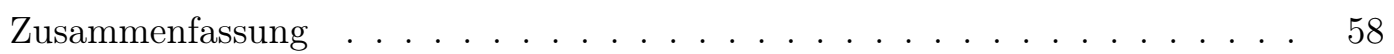

5 Strukturelle Eigenschaften und Korrelationen zum Magnetismus 61

5.1 Kristallqualität . . . . . . . . . . . . . . . 62

5.2 Einbauort und Ladungszustand des Gd . . . . . . . . . . . . . . . . . . . . 63

5.2.1 Röntgenfluoreszens und -absorption . . . . . . . . . . . 63

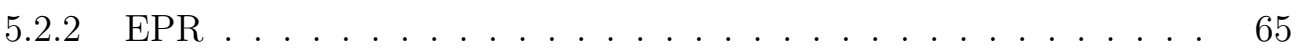

5.3 Positronenvernichtung . . . . . . . . . . . . . . 66

5.4 Optische Eigenschaften . . . . . . . . . . . . . . . . . 68

5.4 .1 Übersichtsspektren . . . . . . . . . . . . . . . . . . . . . . . . . . 69

5.4 .2 Bandkantennahe Lumineszenz . . . . . . . . . . . . . . . 70

5.4.3 Trends der exzitonischen Lumineszenzen . . . . . . . . . . . . 75

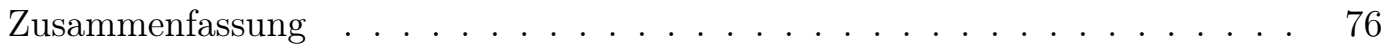

$\begin{array}{ll}\text { Zusammenfassung und Ausblick } & 79\end{array}$

$\begin{array}{ll}\text { A Abbildungen und Tabellen } & 83\end{array}$

$\begin{array}{lr}\text { B Effusion } & \mathbf{8 7}\end{array}$

B.1 Effusion idealer Oberflächen . . . . . . . . . . . . . . . . . 88

B.2 Korrekturen für reale Effusionszellen . . . . . . . . . . . . . . . . . . . . . . . . . . . . . . 89

B.3 Verwendete Zellen und deren Flüsse . . . . . . . . . . . . . . . . . 91

$\begin{array}{ll}\text { C Positronenvernichtung } & 93\end{array}$

C.1 Lebensdauerspektroskopie . . . . . . . . . . . . . . . . . . . . . . . 94 
C.2 Dopplerverbreiterungsspektroskopie . . . . . . . . . . . . . . . . . . . 95

$\begin{array}{lr}\text { D Kalibrierung der Oberflächentemperatur } & 97\end{array}$

D.1 Der Probenhalter . . . . . . . . . . . . . . . . . . . . . . . . . 98

D.2 Pyrometer . . . . . . . . . . . . . . . . . . . . . . . . . 98

D.3 Nachvollziehen der alten Kalibrierung . . . . . . . . . . . . . . . . 99

D.3.1 Si Substrat . . . . . . . . . . . . . . . . . . . . . . . . . 99

D.3.2 Saphir/GaN Substrat . . . . . . . . . . . . . . 100

D.4 Neue Maske . . . . . . . . . . . . . . . . . . . . . . . . . . . . . 101

D.4.1 Si Substrat . . . . . . . . . . . . . . . . . . . 101

D.4.2 Saphir/GaN Substrat . . . . . . . . . . . . . . . 102

D.4.3 SiC Substrat . . . . . . . . . . . . . . . . . . . . 104

D.5 Messtabellen . . . . . . . . . . . . . . . . . 106

$\begin{array}{lr}\text { Literaturverzeichnis } & 109\end{array}$

$\begin{array}{ll}\text { Danksagungen } & 117\end{array}$

$\begin{array}{ll}\text { Lebenslauf } & 119\end{array}$ 


\section{Abbildungsverzeichnis}

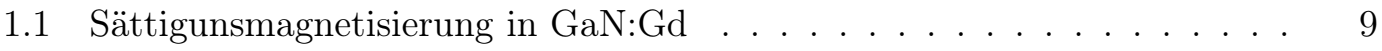

1.2 Kolossale Momente in GaN:Gd durch Verunreinigungen . . . . . . . . 10

1.3 Zustandsdichte und p-d-Wechselwirkung in GaN:Gd . . . . . . . . . . . 14

1.4 Bildungsenergien in- und extrinsischer Defekte in GaN . . . . . . . . . 16

1.5 Skizze der Austauschwechselwirkung in GaN: $\mathrm{V}_{\mathrm{Ga}}$ nach Dev et al. . . . . 18

1.6 Gleichgewichtskonzentration der Galliumleerstelle $\mathrm{V}_{\mathrm{Ga}}$. . . . . . . . . . 20

2.1 Anlagenskizze . . . . . . . . . . . . . . . . . . . . . . 24

2.2 Probenstruktur . . . . . . . . . . . . . . . . . 26

2.3 Abhängigkeiten der Wachstumsrate . . . . . . . . . . . . . . . . . 30

3.1 TRIM Simulation . . . . . . . . . . . . . . . . . . . 32

3.2 Gd Standard TOF-SIMS Messung und Vergleich mit Simulation . . . . . 33

3.3 Gd Tiefenprofil . . . . . . . . . . . . . . . . . . . 37

3.4 Abhängigkeit der Gd Konzentration von der Wachstumsrate . . . . . . . 37

3.5 Gadoliniumkonzentration . . . . . . . . . . . . . . . . 38

3.6 Verunreinigungen in GaN:Gd . . . . . . . . . . . . . . . 40

4.1 Beispiel einer trapped field „Hysterese“ . . . . . . . . . . . . . . . . . 45

4.2 Sättigungsmagnetisierung gegen die Probennummer . . . . . . . . . . . 47

4.3 Feld- und Temperaturabhängigkeit der Magnetisierung der Probe J0176 49

4.4 Vergleich der Hysteresen von nicht co-dotiertem GaN:Gd . . . . . . . . 50

4.5 Vergleich der Hysteresen von co-dotiertem GaN:Gd mit Si oder H . . . . 51

4.6 Vergleich der Hysteresen von Sauerstoff-Co-dotiertem GaN:Gd . . . . . . 52

4.7 Übersicht über die absoluten und effektiven Sättigungsmagnetisierungen 53

4.8 Temperaturabhängige Magnetisierung der Probe J0174 . . . . . . . . . . 55

4.9 XRD $\theta-2 \theta$-Messung der Probe J0174: GdN Ausscheidungen . . . . . . 56

4.10 Zeitabhängigkeit der Sättigunsmagnetisierung ausgesuchter Proben . . . 57

5.1 XRD $\theta-2 \theta$-Messung der Schicht J0175 . . . . . . . . . . . . . . . 62

5.2 Hochaufösende TEM Aufnahmen der Probe J0188 . . . . . . . . . . . 63

5.3 Röntgenfluoreszenz (XRF) und Röntgenabsorption (XANES) . . . . . . 64 
5.4 EPR-Messungen der Schicht J0252 . . . . . . . . . . . . . . . . . 65

5.5 SW-Plot und Tiefenprofile verschiedener GaN:Gd Schichten . . . . . . . 67

5.6 Übersicht über die PL-Spektren . . . . . . . . . . . . . . . . . . . . . 71

5.7 Übersicht über die bandkantennahe Photolumineszens . . . . . . . . . . 72

5.8 Bandkantennahe Lumineszens von GaN:Gd:O . . . . . . . . . . . . . . 74

5.9 Abhängigkeit der bandkantennahen Lumineszens von der Gd Konzentration 75

A.1 SIMS Auswertungsprozedur des Standard . . . . . . . . . . . . . . 83

A.2 SIMS Auswertungsprozedur der Proben . . . . . . . . . . . . . 84

B.1 Zellengeometrie . . . . . . . . . . . . . . . . . . . . . . . . . . . 89

B.2 Teilchenflüsse der Effusionszellen . . . . . . . . . . . . . . . . . . . 92

C.1 Versuchsskizze PAS . . . . . . . . . . . . . . . . . . . . . . . . . 94

D.1 Probenhalter mit alter Maske . . . . . . . . . . . . . . . . . 99

D.2 Temperaturkurve alte Maske mit Si . . . . . . . . . . . . . . . . 100

D.3 Temperaturkurve alte Maske mit Saphir/GaN . . . . . . . . . . . . . 101

D.4 Temperaturkurve neuen Maske mit Si . . . . . . . . . . . . . . . . . . 102

D.5 Rückplatte unpoliert und poliert . . . . . . . . . . . . . . . . 103

D.6 Temperaturkurve neuen Maske mit Saphir/GaN Substrat, neuen Rück-

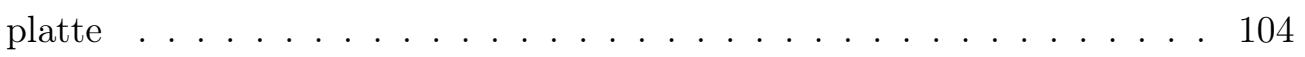

D.7 Temperaturkurve neuen Maske mit Saphir/GaN Substrat, neuen Rückplatte, poliert . . . . . . . . . . . . . . . 105

D.8 Temperaturkurve neuen Maske mit SiC Substrat . . . . . . . . . . 105 


\section{Tabellenverzeichnis}

Vorbehandlung der Substrate, in Abbildung $2.2 \ldots \ldots$. . . . . . . . . . . 26

2.1 Übersicht Wachstumsparameter . . . . . . . . . . . . . . . . . . 27

3.1 Gd Isotope und Masseninterferenzen . . . . . . . . . . . . . . . . . . 34

A.1 Übersicht über die Proben der Serie 1 . . . . . . . . . . . . . . 85

A.2 Übersicht über die Proben der Serie $2 \ldots \ldots$. . . . . . . . . . . 86

B.1 Parameter für die Abschätzung der Teilchenflüsse . . . . . . . . . . . . . 91

D.1 alte Maske. . . . . . . . . . . . . . . . . . . . . 106

D.2 Schmelzpunkte . . . . . . . . . . . . . . . . . 106

D.3 neue Maske . . . . . . . . . . . . . . . . . . . . . . 107 


\section{Abkürzungsverzeichnis}

2DEG two-dimensional electron gas

AES auger electron spectroscopy

BEP beam equivalent pressure

CCD charge coupled device

DAP donor acceptor pair

DFT density functional theory

DMS dilute magnetic semiconductor

ELSA Epitaxie, LEED und Spektroskopie Anlage

EPR electron paramagnetic resonance

ESRF Electron Syncrotron Radiation Facility

EXAFS extended $x$-ray absorption fine spectroscopy

fc field cooled

FIB focused ion beam

GMR giant magneto resistance

LEED low electron energy diffraction

LSDA local spin density approximation

MBE molecular beam epitaxy

MC monte carlo

MOCVD metal organic chemical vapor deposition

MRAM magnetic random access memory

PAS positron annihilation spectroscopy

PL photoluminescence

RE rear earth

RHEED reflection high electron energy diffraction

$\mathrm{RL}$ red luminescence

SIMS secondary ion mass spectroscopy

SQUID superconducting quantum interference device

TEM transmission electron microscopy 
TES two electron satellite

TM transition metal

TOF-SIMS time of flight secondary ion mass spectroscopy

UHV ultra high vacuum

VRH variable range hopping

wz wurzit

XANES $x$-ray absorption near edge spectroscopy

XLD $x$-ray linear dichroism

XMCD $x$-ray magnetic circular dichroism

XPS $x$-ray photon spectroscopy

XRD $x$-ray diffraction

XRF $x$-ray fluorescence

$\mathrm{YL}$ yellow luminescence

zb zincblende

zfc zero field cooled 


\section{Einleitung}

Mit der Entdeckung des GMR-Effektes (giant magneto resistance) durch Grünberg und Fert im Jahr 1988 wurde ein wichtiger Grundstein für die Spinelektronik (kurz: Spintronik) gelegt $[1,2]$. Die Autoren entdeckten, daß der elektrische Widerstand über einen Schichtstapel aus ferromagnetischen und nicht ferromagnetischen Metallen stark von von der Orientierung der ferromagnetischen Schichten zueinander abhängt und wurden später dafür mit dem Nobelpreis ausgezeichnet. Die prominentesten Beispiele für Bauelemente, welche den GMR-Effekt nutzen sind Leseköpfe von Festplatten, Magnetfeldsensoren und der MRAM (magnetic random access memory).

Auch im Bereich der Halbleitertechnologie hat die (Halbleiter-) Spintronik in den vergangenen Jahren beachtliches Interesse erfahren. Mit der Hoffnung den Spin in nur aus Halbleitern aufgebauten Bauelementen zu manipulieren, entstand der Bedarf an Halbleitermaterialien mit magnetischen Eigenschaften. Man verspricht sich daraus die Möglichkeit Bausteine für schnellere, effizientere oder nicht-flüchtige Bauelemente zu konstruieren, welche sich besser in bestehende Halbleitertechnologie integrieren lassen als klassische GMR Elemente.

Verdünnte magnetische Halbleiter (DMS - dilute magnetic semiconductors) sind Halbleitermaterialien, in welchen eines der Matrixelemente gegen ein magnetisches Ion ersetzt wird. Üblicherweise sind diese magnetischen Dotierungen Elemente der Übergangsmetalle (TM) oder der seltenen Erden (RE) und ersetzen das Kation in III-V- oder II-VIHalbleitern. Um kommerziell verwertbare Bauelemente zu konstruieren werden DMS benötigt, welche eine magnetische Ordnung mit einer Übergangstemperatur deutlich oberhalb der Raumtemperatur zeigen und die Eigenschaft besitzen, effizient den Spin der Ladungsträger zu injizieren, manipulieren oder transferieren. Diese Voraussetzung hat sich als eine der größten Herausforderungen für die DMS Forschung herausgestellt. Vor kurzem schlossen Zunger et al. einen Review Artikel über Irrungen der Theorie im Bezug auf DMS mit der Frage „Bad is good?“ [3]. Dabei bezog sich diese Aussage auf die Kristallinität, die Verunreinigungen und Defektdichten in bei Raumtemperatur ferromagnetischen Oxid-DMS. Für Materialien wie z.B. ZnO:TM konnte gezeigt werden, daß die magnetische Kopplung nur in als schlecht im Sinne z.B. der Optoelektronik zu beschreibendem Material auftritt. Hier wird deutlich, daß für eine erfolgreiche Implementation von DMS Materialien in die Halbleitertechnologie eine exakte Steuerung und nicht eine komplette Reduktion von Defekten enorme Bedeutung für die DMS-Forschung hat. 
Unter den angesprochenen Oxid-DMS ist besonders $\mathrm{ZnO}$ :Co mit einer Curietemperatur von ca. $350 \mathrm{~K}$ viel versprechend [4], aber auch andere Halbleitersysteme zeigen gute Kandidaten. Unter den III-V-Halbleitern sind besonders GaAs:TM und GaN:TM erforscht. Aber auch AlN oder InN mit TM oder RE dotiert zeigen magnetische Eigenschaften. Am besten verstanden sind unter den III-V-Halbleitern die Eigenschaften des GaAs:Mn. Weitere wichtige Systeme sind GaN:Fe und GaN:Gd. Der erste Bericht über ferromagnetisches Verhalten von GaN:Gd erschien im Jahre 2002 von Teraguchi et al. [5]. Es wurden GaN-Schichten auf $\mathrm{SiC}$ mit Molekularstrahlepitaxie (MBE - molecular beam epitaxy) hergestellt, in welche während des Wachstums ca. $6 \%$ Gd dotiert wurden. Das Material zeigt selbst bei $400 \mathrm{~K}$ ferromagnetisches Verhalten und hat ein Koerzitivfeld von 70 Oe (bei $300 \mathrm{~K}$ ). Nachdem zwei folgende Arbeiten der gleichen Arbeitsgruppe weitere Beweise für einen kollektiven Effekt in GaN:Gd zeigten [6, 7] erschien die wohl am stärksten diskutierte Veröffentlichung über GaN:Gd von Dhar et al.. Stark verdünnte GaN:Gd-Schichten mit Gd-Konzentrationen im Bereich von $10^{16}-10^{19} \mathrm{~cm}^{-3}$ zeigten ferromagnetisches Verhalten bei Raumtemperatur [8]. Dieses ist eine bemerkenswerte Eigenschaft, da DMS wie GaAs:Mn oder GaN:Mn Verunreinigungskonzentrationen von mehreren Prozent benötigen, um eine ferromagnetische Ordnung auf makroskopischer Skala auszubilden und dabei im Falle von GaAs:Mn nur Curietemperaturen von ca. $170 \mathrm{~K}$ erreicht werden. Über dies hinaus fanden Dhar et al. in ihren Schichten ein effektives magnetisches Moment pro Gd-Verunreinigung von bis zu $10^{5} \mu_{\mathrm{B}}$ und damit ein weit höheres Moment als das atomare Moment des Gadoliniums von $8 \mu_{\mathrm{B}}$. Sie nannten dieses Verhalten kolossale magnetische Momente und schlossen, daß eine Polarisierung der GaN-Matrix zu diesem Effekt führt. Es wurde ein phänomenologisches Modell vorgeschlagen, welches die effektive Magnetisierung gut beschreibt (Abschnitt 1.2). Darauf folgten zahlreiche theoretische und experimentelle Arbeiten, welche verschiedene Ansätze für die bis heute nicht verstandenen Effekte in GaN:Gd liefern.

Der Gedanke seltene Erden in III-V-Halbleiter zu dotieren, um einen verdünnten magnetischen Halbleiter zu erhalten, birgt viele Ideen. Aus der Optoelektronik ist das Dotieren mit seltenen Erden schon länger bekannt. Die sehr scharfen Übergänge der stark lokalisierten 4f-Zustände bieten interessante Möglichkeiten für die Erzeugung von monochromatischem Licht. Auch für den Anwendungsbereich der DMS bieten sie viel versprechende Eigenschaften. Die partiell gefüllte 4f-Schale bringt im allgemeinen ein höheres atomares Moment mit sich als die 3d-Zustände der Übergangsmetalle. Besonders die Dotierung von GaN mit Gd erscheint hier interessant. Gd hat die Elektronenkonfiguration $[X e] 4 f^{7} 5 d^{1} 6 s^{2}$ und trägt ein atomares Moment von $8 \mu_{\mathrm{B}}$. Nimmt man an, daß Gd auf dem Ga-Untergitter in GaN eingebaut wird, so liegt es (wie Ga) im dreifach geladenen Zustand vor. Es ist damit isovalent zu Ga und bringt immer noch $7 \mu_{\mathrm{B}}$ aus den ungepaarten Spins der halb gefüllten 4f-Schale mit. Atomares Gd zeigt eine große Austausch-Aufspaltung der 4f-Zustände von ca. $12 \mathrm{eV}$ [9], die Lage der 4f-Zustände kann 
also außerhalb der Bandlücke erwartet werden. Der erste angeregte Zustand des $\mathrm{Gd}^{3+}$ $\left({ }^{6} \mathrm{P}_{7 / 2}\right)$ liegt ca. $3,9 \mathrm{eV}(318 \mathrm{~nm})$ über dem Grundzustand $\left({ }^{8} \mathrm{~S}_{7 / 2}\right)$ und ist damit energetisch oberhalb der Bandlücke von GaN (3,4-3,5eV) angesiedelt [10]. Eine isovalente Verunreinigung ohne erwartete Zustände innerhalb der Bandlücke in GaN einzubringen verspricht sehr interessante elektrische Eigenschaften. GaN ist typischerweise n-leitend aufgrund von Restverunreinigungen (Sauerstoff) und intrinsischen Defekten und sollte von Gd nicht beeinflußt werden. Auf den ersten Blick ist GaN:Gd also ein guter Kandidat für einen n-leitenden verdünnten magnetischen Halbleiter, welcher alle Eigenschaften für die Spininjektion in reinen Halbleiter Systemen mit sich bringen sollte.

Die vorliegende Arbeit untersucht vorwiegend die Punkte Herstellung (Kapitel 2), Gd-Konzentration und Verunreinigungen (Kapitel 3), Ferromagnetismus bei Raumtemperatur, Reproduzierbarkeit und Alterung (Kapitel 4). Jedes der Kapitel sammelt dabei Hinweise auf den Ursprung der magnetischen Eigenschaften. Kapitel 5 nimmt abschließend diese Hinweise auf und versucht diese in gezielter Weise mit dem Magnetismus zu korrelieren. Als Grundlage dafür liefert Kapitel 1 eine ausführliche Diskussion der Veröffentlichungen über GaN:Gd. 


\section{Kapitel 1}

\section{GaN:Gd - Ein Verdünnter Magnetischer Halbleiter?}

Ist GaN:Gd ein verdünnter magnetischer Halbleiter? Diese Frage stellt sich insbesondere im Bezug auf Erfahrungen mit gut erforschten DMS-Systemen wie GaAs:Mn, GaN:Mn, GaN:Fe oder SnO:Co. Allgemein kann festgestellt werden, daß bei kaum einem der vermeintlichen DMS-Materialien die magnetische Ordnung ausschließlich auf die Dotierung mit einer magnetischen Verunreinigung zurückzuführen ist. Stichworte sind hier Themen wie die spinodale Entmischung, die Bildung magnetischer Ausscheidungen und geblockter Superparamagnetismus. In GaAs:Mn ist die ferromagnetische Kopplung stark von der intrinsischen Defektlandschaft und der Ladungsträgerkonzentration abhängig [11, 12, 13]. GaN:Mn zeigt Tendenzen zweite Phasen mit ausgeprägten magnetischen Eigenschaften zu bilden [14] und in GaN:Fe sind nanoskalige Ausscheidungen für die magnetischen Eigenschaften verantwortlich [15]. Auch überhöhte effektive Momente der magnetischen Verunreinigung sind in verschiedenen DMS, wie SnO:Co zu finden [16]. Für alle diese Effekte gibt es auch Hinweise in GaN:Gd, es ist aber bis heute kein klares Bild über die Herkunft der magnetischen Ordnung gefunden.

Dieses Kapitel soll Hinweise und Fakten über GaN:Gd zusammenfassen und diskutieren. Abschnitt 1.1 stellt dazu wichtige experimentelle Ergebnisse über GaN:Gd zusammen und Abschnitt 1.2 diskutiert insbesondere das Thema der kolossalen Momente. Die Abschnitte 1.3 und 1.4 fassen die wichtigsten theoretischen Modelle im Bezug auf magnetische Ordnung in GaN:Gd zusammen. Die darauf folgenden Abschnitte beschäftigen sich mit dem Bild verschiedener Defekte und deren Rolle für den Magnetismus. Abschließend wird das Kapitel zusammengefaßt. 


\subsection{Strukturelle, magnetische und elektrische Eigenschaften}

Die Herstellung von Gd-dotiertem GaN präsentiert sich in der Literatur als unproblematisch. Verschiedene Arbeiten zeigen, daß für die Herstellung von bei Raumtemperatur ferromagnetischem GaN:Gd nur geringe Gd-Konzentrationen unterhalb eines Prozents nötig sind [17, 18, 19]. Diese Eigenschaft macht GaN:Gd zu einem viel versprechenden DMS-System, da bei geringen Dotierungen auch geringe Einflüsse auf Wachstumseigenschaften erwartet werden können.

Dhar et al. zeigen in ihrer Wachstumsstudie mit RHEED (reflection high electron energy diffraction), daß das Wachstum auf SiC Substraten mit Ammonia-MBE dem von undotiertem GaN entspricht [17]. Für das Wachstum mit Stickstoffplasma-unterstützter MBE auf MOCVD-GaN-Templates (metal organic chemical vapor deposition) zeigen Hite et al., daß bis zu einer kritischen Dicke von ca. $150 \mathrm{~nm}$ das Wachstum im zweidimensionalem Wachstumsmodus stattfindet, dann aber zu einem dreidimensionalen übergeht [18]. Beide Gruppen zeigen darüber hinaus anhand von XRD (x-ray diffraction) und TEM (transmission electron microscopy) Daten, daß die Gd-Dotierung keinen Einfluß auf die Schichtqualität des GaN hat und keine Ausscheidungen bis zu einer Gd-Konzentration von ca. $10^{19} \mathrm{~cm}^{-3}$ zu finden sind.

Über den Einbauort des Gd in der GaN-Matrix und seinen Ladungszustand geben XANES- ( $x$-ray absorption near edge structure) und XLD-Messungen ( $x$-ray linear dichroism) Auskunft. Ney et al. bestätigen den vorwiegenden Einbau des Gd auf dem GaUntergitter [20]. Auch an den im Rahmen dieser Arbeit hergestellten Schichten konnten Martínez-Criado et al. den Einbau auf einem Ga-Platz bestätigen. Das Gd liegt vorwiegenden im Ladungszustand 3+ vor [21].

Höhere Gd-Konzentrationen wurden von Zhou et al. realisiert [7, 22, 23]. GaN:Gd mit Gd-Konzentrationen von bis zu 12,5\% wurden bei tiefen Substrattemperaturen von $\mathrm{T}_{\mathrm{S}}$ $=300^{\circ} \mathrm{C}$ bis zu Raumtemperatur direkt auf Saphir mit Stickstoffplasma-unterstützter MBE hergestellt. XRD und XANES Messungen zeigen, daßs so Gd-Konzentrationen von bis zu 12,5\% ohne Ausscheidungen möglich sind. Aus XANES- und PL-Messungen ( $p h o-$ toluminescence) wird weiter auf eine hohe Anzahl von Stickstoffleerstellen (Donatoren) geschlossen.

Alle Proben der Arbeitsgruppen um Dhar, Hite und Zhou zeigen ferromagnetisches Verhalten bei Raumtemperatur [8, 18, 7]. Dhar et al. heben dabei die effektive Magnetisierung pro Gd-Atom hervor, welche in den Schichten bis zu $4000 \mu_{\mathrm{B}}$ erreichen (Abschnitt 1.2). Hite etal. und Zhou etal. machen keine Aussagen über die effektive Magnetisierung. Beide Gruppen untersuchen den Einfluß einer Si-co-Dotierung auf die magnetischen Eigenschaften. Hite et al. zeigen, daß die magnetischen Eigenschaften mit der Si-Konzentration bzw. der Ladungsträgerkonzentration skalieren. Niedrige SiKonzentrationen führen zu einer geringeren Sättigungsmagnetisierung und erst in hoch 
Si-dotiertem GaN:Gd werden wieder vergleichbare Sättigungsmagnetisierungen erreicht wie in undotiertem GaN:Gd. Zhou et al. zeigen für sehr hohe Gd-Konzentrationen um die $10 \%$ hingegen eine starke Erhöhung der Sättigungsmagnetisierung mit der co-Dotierung von $\mathrm{Si}$.

Auch wenn für keine der Proben von Dhar et al. Ausscheidungen nachgewiesen werden konnten gibt es doch Hinweise auf eine solche zweite Phase. In allen der Schichten tritt ein magnetischer Übergang bei ca. $70 \mathrm{~K}$ auf, welcher in temperaturabhängigen Messungen mit SQUID (superconducting quantum interference device) zu sehen ist [17]. Dieser Übergang ist auch in Schichten von Lo et al. zu finden und es wird auf superparamagnetische geblocktes Verhalten geschlossen [24]. Im Rahmen dieser Arbeit konnte der Übergang mit GdN-Ausscheidungen korrelierte werden (Abschnitt 4.7).

Die elektrischen Eigenschaften von GaN:Gd sind deutlich schwieriger zu interpretieren. Schon für kleine Gd Konzentrationen zeigen die Schichten von Dhar et al. und Hite et al. hochohmiges Verhalten. Auch für das Material dieser Arbeit konnten Bedoya-Pinto et al. zeigen, daß GaN bei der Zugabe von $2 \cdot 10^{16} \mathrm{~cm}^{-3}$ Gd hochohmig wird und VRHLeitfähigkeit (variable range hopping) in einem schmalen Band lokalisierter Zustände aufweist [25]. Diese Art von Verhalten ist zunächst überraschend, da $\mathrm{Gd}^{3+}$ auf dem Ga-Untergitter isovalent zu Ga ist und somit elektrisch nicht aktiv sein sollte. Auch ist die Kompensation der typischen Hintergrundkonzentration an Ladungsträgern (Elektronen) von ca. $10^{17} \mathrm{~cm}^{-3}$ [25] schwer mit einer Gd-Konzentration der stark verdünnten GaN:Gd-Schichten von $10^{15}-10^{16} \mathrm{~cm}^{-3}$ zu erklären. Si“ co-dotierte Schichten von Hite et al. zeigen n-Leitfähigkeit [18].

Zusammenfassend kann man über die mit MBE hergestellten GaN:Gd-Schichten sagen, daß alle Arbeitsgruppen über ferromagnetisches Verhalten bei Raumtemperatur berichten. Die Magnetisierung zeigt dabei verschiedene Abhängigkeiten von der Ladungsträgerkonzentration und es existieren Berichte über leitfähiges und semi-isolierendes Verhalten. Der genaue Zusammenhang mit der Ladungsträgerkonzentration ist dabei unbekannt. Si-co-Dotierungen und hohe Stickstoffleerstellenkonzentrationen bei Hite et al. und Zhou et al. weisen dabei auf einen Ladungsträger-induzierten Kopplungsmechanismus hin, wobei Stickstoffleerstellen auch auf die Einflußnahme von Defekten deuten kann.

Zusätzlich zu dem mit MBE hergestellten Material gibt es Berichte über in GaN implantiertes Gd. Dhar et al. und Khaderbad et al. [26, 27] zeigen, daß für die Implantation von Gd-Ionen mit einem FIB (focused ion beam) in GaN in der Wurzitstruktur (wz) effektive Sättigungsmagnetisierungen bei Raumtemperatur erreicht werden, die jene von MBE-hergestelltem GaN:Gd noch um eine Größenordnung übersteigen. Die Schichten haben damit eine Sättigungsmagnetisierung von ca. $10^{5} \mu_{\mathrm{B}} / \mathrm{Gd}$. Alle Schichten wurden bei unterschiedlichen Temperaturen ausgeheizt, wobei die höchsten Magnetisierungen für nicht ausgeheilte Schichten erreicht wurden. Geschlossen wird mit der Vermutung, 
daß eine Polarisation der Ga-Matrix oder Stickstoff- oder Galliumleerstellen, welche bei der Implantation entstehen, für die kolossalen Momente verantwortlich sein müssen. Es wird weiter vermutet, daß diese auch durch das Gd induziert beim MBE-Wachstum entstehen. Lo et al. berichten über die Implantation in GaN-Schichten mit Wurzit- und Zinkblende-Struktur (zb) [24, 28]. Keine der Proben wurde nach der Implantation einem Ausheilprozess unterzogen. Für Schichten mit zb-Struktur wird kein ferromagnetisches Verhalten bei Raumtemperatur gefunden. Eine superparamagnetische Signatur unterhalb von ca. $60 \mathrm{~K}$ läßt auf GdN-Ausscheidungen schließen. Im Falle des wz-GaN wird das Gd in eine Heterostruktur mit zweidimensionalem Elektronengas (2DEG) implantiert. Das 2DEG bleibt leitfähig nach der Implantation von Gd und es wird der anormale Halleffekt beobachtet. Lo et al. schließen hieraus auf einen Einfluß der im wz-GaN vorliegenden spontanen Polarisationsfelder auf die magnetischen Eigenschaften von GaN:Gd. Han et al. zeigen die Implantation von Gd in GaN- und AlN-Schichten und anschließende Ausheilschritte [29]. Beide zeigen Ferromagnetismus bei Raumtemperatur, wobei die höchsten Magnetisierungen für Ausheiltemperaturen um die $900^{\circ} \mathrm{C}$ erreicht wurden. Hier wird geschlossen, daß für niedrigere Temperaturen die Implantationsschäden nicht ausgeheilt sind.

Zusammenfassend ist über Gd-implantiertes GaN zu sagen, daß die Ergebnisse der verschiedenen Ausheizexperimente widersprüchlich sind. Mit der Arbeit von Lo et al. werden auch die einzigen Ergebnisse gezeigt, die keine magnetische Ordnung bei Raumtemperatur zeigen. Alles in allem wird nach dieser Betrachtung deutlich, daß eine exakte Betrachtung der Defekte und die äußerst genaue Kontrolle der Wachstumsparameter von grundlegender Wichtigkeit für die Identifizierung des Kopplungsmechanismus in GaN:Gd sind.

\subsection{Kolossale Momente und deren Herkunft}

Die von Dhar et al. [8] gefundenen und im Rahmen dieser Arbeit bestätigten ([19], Kapitel 4) kolossalen Momente können alleine durch die Anwesenheit von Gd in der GaNSchicht nicht ausreichend erklärt werden. Abbildung 1.1 zeigt die Auftragung der Sättigungsmagnetisierung gegen die Gd-Konzentration in der GaN-Schicht aus [17]. Schon bei einer geringen Gd-Konzentration von $10^{16} \mathrm{~cm}^{-3}$ zeigen die Schichten ferromagnetisches Verhalten. Rechnet man die absolute Magnetisierung in eine effektive pro Gd-Atom in $\mu_{\mathrm{B}}$ um, so erhält man die Auftragung im Inset von Abbildung 1.1. Für die kleinste Gd-Konzentration werden hier ca. $4000 \mu_{\mathrm{B}} /$ Gd bei einer Temperatur von $2 \mathrm{~K}$ erreicht. In einer späteren Arbeit von Dhar et al. werden für implantiertes Gd in GaN sogar effektive Magnetisierungen von ca. $10^{5} \mu_{\mathrm{B}} / \mathrm{Gd}$ erreicht [26]. Dhar et al. schlagen ein phänomenologisches Model vor, welches den Fit in Abbildung 1.1 liefert. Dieses Model spiegelt zwar 


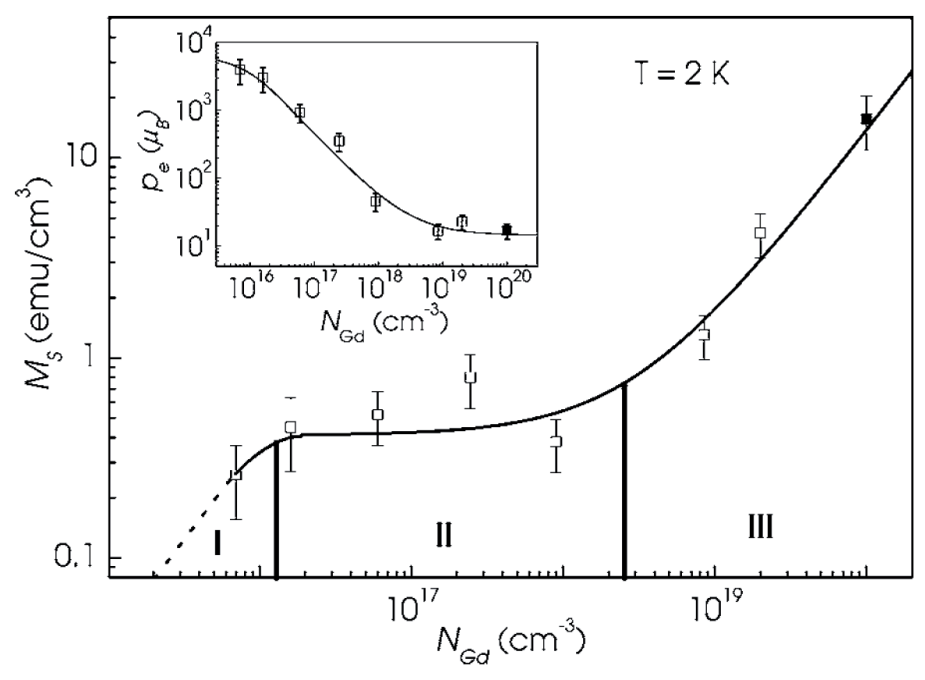

Abbildung 1.1: Sättigungsmagnetisierung in GaN:Gd von Dhar et al. [17].

gut die gemessenen Werte wieder, ist aber als Erklärung für die mikroskopische Ursache der kolossalen Momente nicht ausreichend.

\subsubsection{Phänomenologisches Modell von Dhar et al.}

Das phänomenologische Bild von Dhar et al. basiert auf der Annahme, daß jedes GdAtom in einem bestimmten Radius um sich herum die Atome der GaN-Matrix polarisieren kann. Jedes in diesem Einflußbereich liegende Atom der Matrix wird mit einem bestimmten induzierten Moment verbunden, während Atome außerhalb dieser Bereiche kein Moment beitragen. Für sehr geringe Gd-Konzentrationen ist so das effektive Moment pro Gd maximal (Bereich I in Abbildung 1.1). Steigt die Gd-Konzentration an, so beginnen die Einflußbereiche sich zu überlappen. Jedes Atom der Matrix, welches innerhalb von mehreren Einflußbereichen liegt trägt jetzt ein weiteres Moment, abhängig von der Anzahl der überlappenden Einflußbereiche. Das effektive Moment pro Gd-Atom sinkt in diesem mit II gekennzeichneten Bereich mit steigender Gd-Konzentration. Der Übergang zu Bereich III markiert den Punkt ab welchem die gesamten Atome der GaNMatrix polarisiert sind. Ab hier ist die effektive Magnetisierung pro Gd-Atom konstant nahe dem atomaren Wert von $\mathrm{Gd}\left(8 \mu_{\mathrm{B}}\right)$.

Ney et al. widerlegten eine Polarisation der Ga-Atome durch XMCD-Messungen $(x-$ ray magnetic circular dicroism) [30]. Die Polarisation der Gallium $K$ und $L_{3}$ Kante folgen nicht den Hysteresen, welche im SQUID beobachtet werden konnten. Die Gesamtpolarisation beider Ga-Kanten ist sehr schwach $\left(\approx 10^{-5} \mu_{\mathrm{B}}\right)$ und kann nicht für das beobachtete magnetische Moment in GaN:Gd verantwortlich sein. Auch die Polarisation 


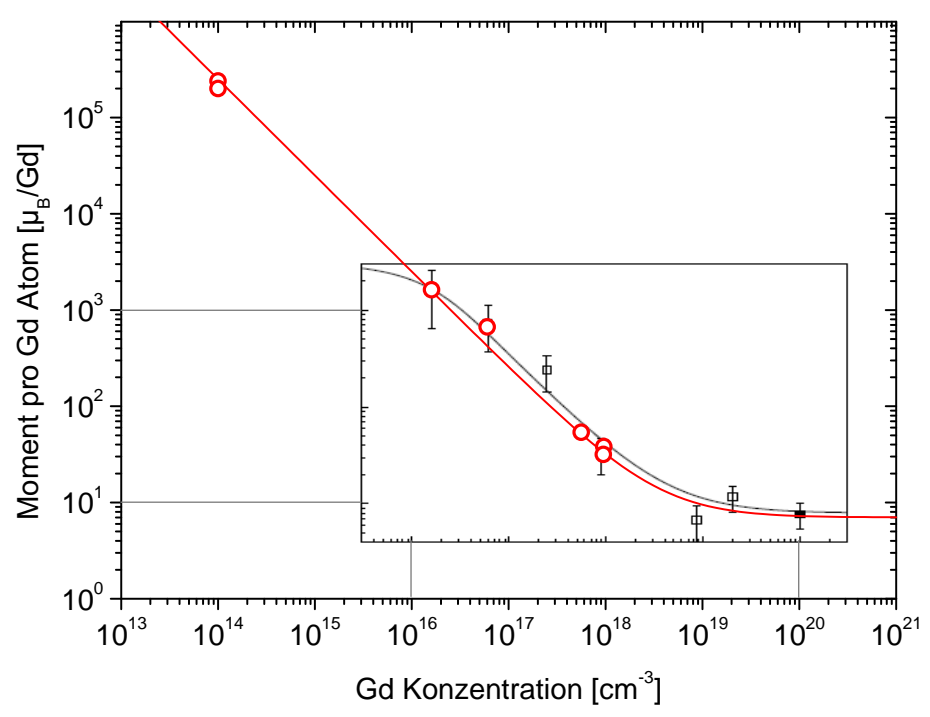

\begin{abstract}
Abbildung 1.2:
Aufgetragen sind die effektiven Sättigungsmomente in $\mu_{\mathrm{B}}$ pro Gd gegen die Gd-Konzentration. Die durchgezogene Linie ist nach Formel 1.1 die erwartete effektive Magnetisierung pro Gd-Atom, wenn eine Verunreinigung mit einer Konzentration von $2,5 \cdot 10^{19} \mathrm{~cm}^{-3}$ für die Magnetisierung der Schicht verantwortlich ist. Der Einsatz zeigt eine Abbildung frei nach [8].
\end{abstract}

der $\mathrm{Gd} \mathrm{L}_{3}$ Kante folgt nicht der integral gemessenen Magnetisierung. Zusammengefaßt zeigt die Arbeit von Ney etal., daß weder das Ga-Atom noch die Gd-Dotierung die magnetischen Momente in GaN:Gd tragen. Alternativ bleiben noch das Stickstoffatom oder andere Defekte innerhalb der Matrix.

\title{
1.2.2 Kolossale Momente durch Defekte
}

Betrachtet man Abbildung 1.1, so kann man auch eine andere Möglichkeit finden, die gemessenen Sättigungsmagnetisierungen von Dhar et al. und auch die in dieser Arbeit beobachteten Ergebnisse zu beschreiben. Dabei handelt es sich zwar auch um eine rein phänomenologische Betrachtung, doch kann eine interessante Abschätzung aus dieser gezogen werden.

Die magnetischen Momente werden von Defekten getragen, welche für sich alleine nicht kollektiv wechselwirken. Die Zugabe von Gd induziert, wie beim phänomenologischen Model von Dhar et al., eine ferromagnetische Wechselwirkung zwischen den Defekten und trägt zusätzlich mit seinen Momenten zur Magnetisierung bei. Nimmt man jetzt weiter an, daß die Konzentration und das Moment der Defekte unabhängig von der Gd-Konzentration und über alle Proben konstant ist, so würden die auf die Gd-Konzentration gerechneten effektiven Momente mit fallender Gd-Konzentration 
ansteigen. Sie werden dann durch folgende Formel beschrieben.

$$
m_{e f f, G d}=m_{G d}+\frac{m_{D e f} \cdot c_{D e f}}{c_{G d}}
$$

$m$ und $c$ sind jeweils das magnetische Moment und die Konzentration des Gd bzw. der Defekte. Trägt man $m_{e f f, G d}$ gegen die Gd-Konzentration auf und paßst Gleichung 1.1 an die Magnetisierungsmessungen von Dhar et al. an, so erhält man die in Abbildung 1.2 gezeigte rote Kurve. Der innere Teil der Abbildung zeigt die effektiven Sättigungsmagnetisierungen von Dhar etal. bei $300 \mathrm{~K}$ und als schwarze Kurve den Fit ihrer Daten nach ihrem phänomenologischen Modell. Rote Kreise zeigen die Sättigungsmagnetisierungen der im Rahmen dieser Arbeit erhaltenen ferromagnetischen Schichten bei $300 \mathrm{~K}$. Für den Fit nach Formel 1.1 (rote Kurve) wurde $m_{G d}$ fest als $7 \mu_{\mathrm{B}}$ gesetzt $\left(\mathrm{Gd}^{3+}\right)$, für $m_{\text {Def }} \cdot c_{\text {Def }}$ ergibt sich dann $2,5 \cdot 10^{19} \mu_{\mathrm{B}} / \mathrm{cm}^{3}$. Bei einem Defekt mit zwei ungepaarten Spins würde eine Defektkonzentration von ca. $10^{19} \mathrm{~cm}^{-3}$ vorliegen. Dieser Wert ist verglichen mit der Gd-Konzentration sehr hoch, aber nicht unrealistisch. In den Arbeiten von Dhar et al. und Hite et al. wird beispielsweise auf eine Sauerstoffkonzentration von $10^{18}$ bzw. $10^{19} \mathrm{~cm}^{-3}$ hingewiesen $[17,31$ ]. Sauerstoff und andere mögliche Defekte werden noch weiter in Abschnitt 1.7 diskutiert.

In diesem einfachen Modell für kolossale Momente durch Defekte ist noch keine Sättigung der effektiven Momente, wie der Bereich I im Modell von Dhar et al. enthalten. Denkbar wäre aber eine Argumentation wie die folgende. Durch den großen Gd-GdAbstand bei sehr verdünnten GaN:Gd-Schichten wird die postulierte langreichweitige Wechselwirkung aufgrund von Perkolationseffekten ineffizient. Dieses wird nicht nur zu einer Sättigung der effektiven Momente pro Gd führen, sondern sogar ein vermutlich steiles Abfallen auf Null für sehr kleine Gd-Konzentrationen zur Folge haben. Auch das phänomenologische Modell von Dhar et al. sollte dieses Verhalten zeigen, da auch dort für kleine Gd-Konzentrationen ein Zusammenbrechen der langreichweitigen Wechselwirkung aus der genannten Argumentation heraus zu erwarten ist. Man kann auch kritisch fragen, ob die beschriebene Sättigung wirklich aus den Daten von Dhar et al. hervorgeht. Wie in Abbildung 1.2 gezeigt, kann die rote Kurve ohne Sättigung im Rahmen der Streuung der Meßwerte den Punkten von Dhar et al. angepaßt werden. Auch die Schichten dieser Arbeit mit Gd-Konzentrationen im Bereich von $10^{14} \mathrm{~cm}^{-3}$ sprechen gegen die von Dhar et al. beobachtete Sättigung der effektiven Momente.

\subsection{Theoretische Modelle}

Mit den theoretischen Modellen verhält es sich ähnlich wie mit den experimentellen Ergebnissen. Sie sind widersprüchlich und zeigen doch für jeden Ansatz ferromagnetisches Verhalten in GaN:Gd. Einige Ansätze führen die Wechselwirkung und die Stabilisierung 
auf Ladungsträger zurück. Hier widersprechen sich die Aussagen von Dalpian und Wei und Liu et al., welche jeweils die Stabilisierung durch Elektronen bzw. Löcher vorhersagen. Weiter gibt es verschiedene Modelle, in denen durch Defekte die ferromagnetische Phase stabilisiert wird. Dev et al. und Godha et al. zeigen, daß die Galliumleerstelle der Grund für das kollektive Verhalten sein kann, Mitra und Lambrecht halten dies für unwahrscheinlich und begründen in ihren Rechnungen den Verdacht auf Stickstoff- oder Sauerstoffzwischengitteratome.

Die einzelnen Arbeiten werden in den folgenden Abschnitten diskutiert. Hier soll noch ein allgemeines Problem der Theorie im Bezug auf GaN:Gd angesprochen werden. Die häufig verwendete local spin density approximation (LSDA) ist für die Beschreibung von unbesetzten und sehr lokalisierten Zuständen, wie den 4f-Zuständen des Gd, und der Bestimmung der Bandlücke von Halbleitern mit großer Bandlücke nur schlecht geeignet. So werden beispielsweise für GaAs $\sim 0,1 \mathrm{eV}$ (experimentell $\sim 1,4 \mathrm{eV}[32]$ ) und für GaN $\sim 2,2 \mathrm{eV}$ (experimentell $\sim 3,5 \mathrm{eV}[33]$ ) gefunden. Interessant sind im Bezug auf dieses Thema die Arbeiten von Svane et al. und Zunger et al. [34, 3].

Für GaAs:Mn ist mit DFT (density functional theory) und statistischen Methoden wie Monte-Carlo-Simulationen (MC) eine gute Beschreibung der magnetischen und elektrischen Eigenschaften möglich [11]. Die experimentell verwirklichten Mn-Konzentrationen sind größer als $1 \%$ und gut mit den theoretischen Methoden zu beschreiben. In GaN:Gd sind die experimentell hergestellten und untersuchten Gd-Konzentrationen deutlich unterhalb von $1 \%$. Selbst große Superzellen mit 576 Atomen, wie bei Gohda et al. verwendet [35], können aber nur Konzentrationen von minimal $\approx 2 \%$ oGd-Konzentration handhaben. Oft sind auch kleinere Superzellen zu finden, welche nur Konzentrationen von mehreren Prozent beschreiben können.

\subsection{Ladungsträger-induzierter Magnetismus}

Die erste theoretische Arbeit zum GaN:Gd nach dem phänomenologischen Modell von Dhar et al. ist die Arbeit von Dalpian und Wei [36]. Mit LSDA-Rechnungen (local spin density approximation) wurde die Bandstruktur von GdN und GaN:Gd mit 12,5\% und $6,25 \%$ Gd-Konzentration berechnet. Es wird für alle Varianten ein halbleitender Charackter vorhergesagt. GdN mit zb-Struktur ist ferromagnetisch, zb-GaN:Gd hat einen antiferromagnetischen Grundzustand. Die 4f- und 5d-Zustände des Gd sind stark spinaufgespalten und liegen innerhalb der Bänder von GaN. Die Bandkante des Minoritätsleitungsbandes wird durch Abstoßung mit den ungefüllten 4f-Bändern in der Energie abgesenkt. Folglich ist die Leitungsbandkante spinaufgespalten, dadurch kann das System bei der Zugabe von Elektronen den ferromagnetischen Zustand stabilisieren. Die Aufspaltung der $4 \mathrm{f}-$ Zustände wird hier zu $4 \mathrm{eV}$ berechnet. Für die von Dhar et al. gefundenen 
kolossalen Momente wird ein Erklärungsansatz geliefert. Die in der Nähe der Leitungsbandkante liegenden 4f-Minoritätszustände können mit den s-artigen Leitungsbandzuständen hybridisieren, da die s-f Hybridisierung Symmetrie-erlaubt ist. Ladungsträger von der Leitungsbandkante oder einem flachen Donator können jetzt diese Zustände bevölkern und so die ferromagnetische Phase stabilisieren. Die effektiven Momente wären dann auf die Ladungsträgerkonzentration zurückzuführen. Dalpian und Wei weisen auch darauf hin, daß eine hohe Sauerstoffkonzentration von Dhar et al. gefunden wurde, welche als Donator in GaN die Theorie des Ladungsträger-induzierten Ferromagnetismusses unterstützt [8]. Das hochohmige Verhalten wird durch die starke Lokalisierung der Elektronen in den 4f-Zuständen nahe der Leitungsbandkante erklärt. Diese stehen dort nicht mehr für die elektrische Leitung zur Verfügung.

Ein Problem dieser Theorie ist, daß selbst bei geringen Dotierungen von ca. $10^{15} \mathrm{~cm}^{-3}$ Gd hochohmige Schichten beobachtet werden [17]. Eine Bevölkerung dieser Zustände mit Ladungsträgern von der Leitungsbandkante oder aus flachen Donatoren ist zwar denkbar, aber bei einer Hintergrundkonzentration von $10^{17} \mathrm{~cm}^{-3}$ Elektronen (üblich in GaN [25]) reichen die vom Gd zur Verfügung gestellten Zustände nicht aus, um die Ladungsträger von der Leitungsbandkante aufzunehmen und somit ein hochohmiges Verhalten zu erzeugen.

Ein vergleichbares Szenario wird auch von Svane et al. für Gd in GaN vorhergesagt [34]. Die Arbeit befaßt sich auf theoretischer Basis mit zb-GaN:Gd. Die elektronischen Zustände von seltenen Erden in GaAs und GaN werden mit einem auf der LSDAMethode basierenden Formalismus berechnet. Die Austauschwechselwirkungen der seltenen Erden in GaAs und GaN zeigen sich um eine Größenordnung kleiner als die von Übergangsmetallen. Es wird geschlossen, daß Gd als alleinige Dotierung nicht für die beobachteten magnetischen Momente, wie z.B. in [26] verantwortlich sein kann. In diesem Punkt stimmen die Arbeiten von Svane et al. und Dalpian und Wei nicht überein.

Auch von Liu etal. schlagen einen Mechanismus vor, welcher über Ladungsträger die ferromagnetische Phase stabilisiert. Der Kopplungsmechanismus ist dabei ähnlich dem in GaAs:Mn [37]. Mit first-principles Rechnungen wird die elektronische Struktur und die Austauschwechselwirkung für wz- und zb-GaN:Gd berechnet. Beide Kristallstrukturen liefern qualitativ gleiche Ergebnisse, was die Vermutung von Svane et al. unterstützt, daß die Kristallstruktur (wz oder zb) einen vernachlässigbaren Einfluß auf den Magnetismus hat. Die stark spinaufgespaltenen 4f-Zustände des Gd liegen tief in den Bändern und die Wechselwirkung dieser Zustände mit freien Ladungsträgern wird als sehr schwach angenommen. Die $5 \mathrm{~d} \mathrm{t}_{2 \mathrm{~g}}$-Zustände liegen, ähnlich wie die $3 \mathrm{~d}$-Zustände des $\mathrm{Mn}$ in GaAs, direkt unterhalb der Valenzbandkante und eine p-d-Hybridisierung mit den Valenzbandkantenzuständen wird als stark berechnet (Abbildung 1.3(a)). Der Energieunterschied $\Delta \mathrm{E}_{\mathrm{FM}-\mathrm{AFM}}$ zwischen ferro- und antiferromagnetischem Grundzustand wird zu Null berechnet und somit ein paramagnetisches Verhalten von GaN:Gd 


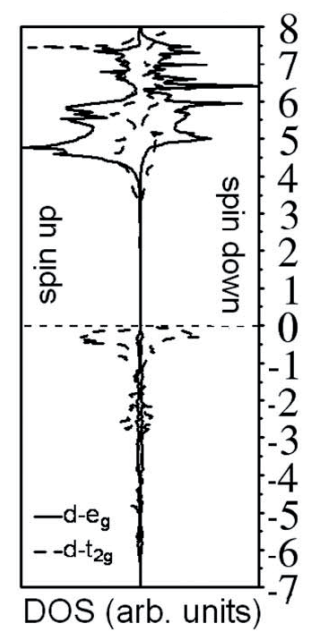

(a) 5d Zustandsdichte

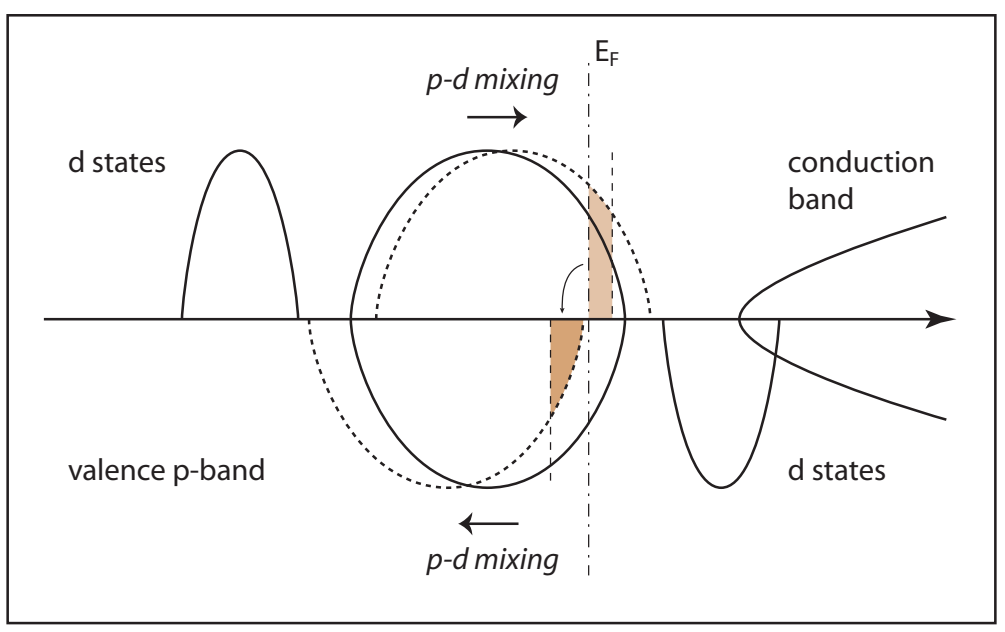

(b) p-d Austausch

\section{Abbildung 1.3:}

(a) zeigt die Zustandsdichte der 5d-Zustände in GaN:Gd aus der Arbeit von Liu et al., (b) skizziert die p-d-Wechselwirkung in GaN:Gd.

vorhergesagt. Der Einfluß von Ladungsträgern (Elektronen und Löchern) wird durch das einbringen von Stickstoff- und Galliumvakanzen $\left(\mathrm{V}_{\mathrm{N}}\right.$ und $\left.\mathrm{V}_{\mathrm{Ga}}\right)$ untersucht, welche als Donator, bzw. Akzeptor agieren (Abbildung 1.4). Die Energiedifferenz $\Delta \mathrm{E}_{\mathrm{FM}-\mathrm{AFM}}$ im Falle von wz-GaN:Gd: $V_{N}$ mit einer Stickstoffleerstelle (Donator) in der Einheitszelle ist $-0,7 \mathrm{meV}$. Es wird also entgegen den Erkenntnissen von Dalpian und Wei [36] vorhergesagt, daß Elektronen nur in vernachlässigbarem Umfang die ferromagnetische Konfiguration begünstigen. Galliumleerstellen sind Akzeptoren und stellen je drei Löcher an der Valenzbandkante zur Verfügung. Die Energiedifferenz $\Delta \mathrm{E}_{\mathrm{FM}-\mathrm{AFM}}$ für GaN:Gd: $\mathrm{V}_{\mathrm{Ga}}$ mit einer Galliumleerstelle in der Einheitszelle ist $-326,1 \mathrm{meV}$. Es wird eine starke p-dWechselwirkung der 5d-Zustände an der Valenzbandkante mit den p-artigen Bindungszuständen gefunden und so eine langreichweitige ferromagnetische Wechselwirkung aufgebaut (Abbildung 1.3(b)). Auch bei teilweiser Kompensation der Löcher durch Donatoren (Sauerstoff) wird diese p-d-Wechselwirkung noch als stark berechnet und führt zu einem ferromagnetischen Zustand. Dieses Szenario kann auch das semi-isolierende Verhalten von GaN:Gd erklären.

Neben leitfähigem GaAs:Mn mit mehreren Prozent Mn Konzentration existieren auch hochohmige Schichten für Mn-Konzentrationen unterhalb und um ein Prozent. Für diese Schichten ist mit der von Liu et al. beschriebenen Art der Kopplung das gleichzeitige Auftreten von Hochohmigkeit und Magnetismus für GaAs:Mn bekannt [13, 12]. Eine starke p-d-Wechselwirkung führt zur Hybridisierung der 3d-Zuständen des Mn mit der 
Valenzbandkante (Abbildung 1.3(b)). Substitutionelles Mangan auf dem Gallium Untergitter in GaAs bildet Akzeptorzustände und stellt so freie Löcher zur Verfügung. Aus dem Vergleich der beiden Materialien wird klar, daß diese Art der Kopplung in GaN:Gd nur stattfinden kann, wenn ein weiterer Defekt, ein Akzeptor, an der ferromagnetischen Kopplung beteiligt ist, da $\mathrm{Gd}^{3+}$ in GaN isovalent $\mathrm{zu}$ Ga ist und selbst keine Löcher für den Kopplungsmechanismus liefern kann. Zusammen mit dem in Abschnitt 1.2.2 vorgestellten Bild der kolossalen Momente durch Defekte könnte so aber das beobachtete Verhalten von GaN:Gd erklärt werden. Die folgenden Abschnitte gehen dazu noch weiter auf Defekte in GaN ein.

\subsection{Defekte und Dotierungen in GaN}

Allgemein wurde aus der bisherigen Betrachtung deutlich, daß Defekte in verschiedener Art und Weise eine Rolle bei den magnetischen und elektrischen Eigenschaften in GaN:Gd spielen könnten. In diesem Abschnitt sollen kurz die gängigsten Defekte und Dotierungen vorgestellt werden, um in den folgenden Abschnitten als Grundlage für die weitere Diskussion zu dienen.

Abbildung 1.4 zeigt die Bildungsenergien von intrinsischen Punktdefekte und ausgesuchten extrinsischen Defekten oder Defektkomplexen in GaN. Aufgetragen ist die Bildungsenergie gegen die Lage der Fermienergie in der Bandlücke von GaN. Die Null der Skala ist auf die Valenzbandkante gelegt und es wurden metallreiche Wachstumsbedingungen angenommen. Die Daten stammen aus den Arbeiten von van de Walle, Neugebauer und Wright [38, 39, 40, 41]. Eine positive Steigung der Kurve deutet auf Donatorverhalten, eine negative Steigung auf Akzeptorverhalten hin. Der Betrag der Steigung entspricht dem Ladungszustand, bezogen auf die ungeladene Störstelle. Änderungen der Steigung entsprechen den Umladungsniveaus des entsprechenden Defektes.

Sauerstoff auf einem Stickstoffplatz $\left(\mathrm{O}_{\mathrm{N}}\right)$ ist ein flacher Donator $(33 \mathrm{meV}$, [40]) und häufig verantwortlich für das n-Typ Verhalten von nominell undotiertem GaN. Eine Hintergrunddotierung mit Sauerstoff im Herstellungsprozess zu vermeiden ist selbst unter UHV-Bedingungen (Ultra Hoch Vakuum) schwierig. Sauerstoffkonzentrationen von $10^{18}-10^{19} \mathrm{~cm}^{-3}$ sind nicht ungewöhnlich und auch in verschiedenen GaN:Gd-Schichten gefunden worden $[17,31]$. $\mathrm{O}_{\mathrm{N}}$ wird auch als mitverantwortlich für die gelbe Lumineszenz (YL) in GaN gehandelt $\left(\mathrm{V}_{\mathrm{Ga}^{-}}-\mathrm{O}_{\mathrm{N}}-\mathrm{Komplex}\right)[38]$.

Interessant ist an dieser Stelle die Frage, ob Gd den Einbau von Sauerstoff in die GaN-Matrix begünstigt und es so zu einem erhöhten Einbau von Sauerstoff durch Gd kommt. Gd ist allgemein leicht zu oxidieren und bildet an der Umgebungsluft schnell eine Oxidschicht. Mitra und Lambrecht errechnen, daß auch in der GaN Matrix die Bildung des Komplexes eines Gd-Ions auf einem Ga-Platz mit einem Sauerstoffzwischengittera- 


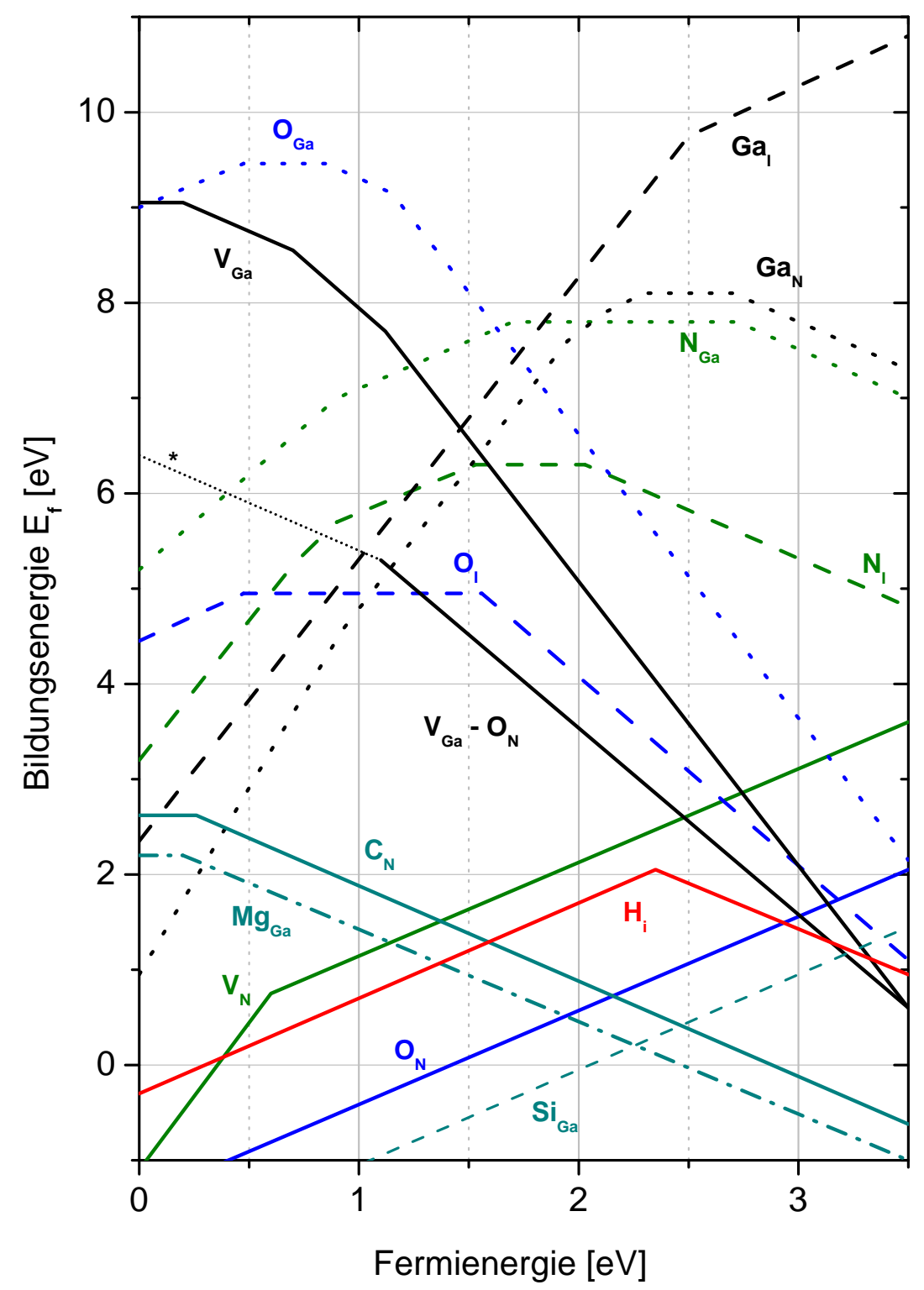

Abbildung 1.4:

Bildungsenergien der wichtigsten intrinsischen Punktdefekte in $\mathrm{GaN}$ und der Dotierungen $\mathrm{Si}, \mathrm{Mg}$, $\mathrm{C}, \mathrm{H}$ und $\mathrm{O}$. Die Bildungsenergie des Komplexes $\mathrm{V}_{\mathrm{Ga}}-\mathrm{O}_{\mathrm{N}}$ ist für den Ladungszustand $2^{-}$und $3^{-}$ eingezeichnet. Im Bereich des zweifach negativen Komplexes $\left(^{*}\right)$ können Übergänge zu Ladungszuständen mit niedrigeren Energien möglich sein. Es wurden metallreiche Wachstumsbedingungen angenommen (außer $\mathrm{MgGa}_{\mathrm{G}}$, für Ga reiche Bedingungen werden höhere Bildungsenergien als die gezeigten erwartet). (aus [38, 39, 40,41]) 
tom $\left(\mathrm{Gd}_{\mathrm{Ga}^{-}} \mathrm{O}_{\mathrm{i}}\right)$ energetisch günstig ist (Bildungsenthalpie kleiner Null) aber keine starke oktaederische Bindung bildet. Die experimentell gefundenen hohen Sauerstoffkonzentrationen unterstützen das Bild eines verstärkten Einbaus von Sauerstoff durch Gd.

Wasserstoff ist in GaN eine amphoterische Verunreinigung. Je nach Lage der Fermienergie verhält sie sich als Akzeptor bzw. Donator und kompensiert so die vorherrschende Ladungsträgersorte. Darüber hinaus ist für Wasserstoff bekannt, daß Akzeptoren passiviert werden können, beispielsweise für den Fall GaN:Mg:H ([38]). Auch Einflüsse auf intrinsische Defekte sind bekannt. So wird z.B. die $\mathrm{V}_{\mathrm{Ga}}$-Konzentration von Wasserstoff reduziert [42].

Neben $\mathrm{O}_{\mathrm{N}}$ ist die Galliumleerstelle $\left(\mathrm{V}_{\mathrm{Ga}}\right)$ in n-Typ GaN zu finden. Besonders für Fermienergien nahe der Leitungsbandkante ist die Bildung dieses akzeptorartigen Defekts günstig (Abbildung 1.4). $\mathrm{V}_{\mathrm{Ga}}$ wird in MBE- oder MOCVD-hergestelltem GaN vorrangig als Komplex mit einem Sauerstoff gefunden [43]. Undekorierte Leerstellen treten nur in durch Beschuß mit hochenergetischen Teilchen beschädigten Kristallen auf. Saarinen et al. zeigen, daß $\mathrm{V}_{\mathrm{Ga}}$ und $\mathrm{V}_{\mathrm{Ga}}-\mathrm{O}_{\mathrm{N}}$ in direktem Zusammenhang mir der $\mathrm{YL}$ in GaN stehen [44].

Als Gegenstück zu $\mathrm{V}_{\mathrm{Ga}}$ ist die Stickstoffleerstelle $\mathrm{V}_{\mathrm{N}} \mathrm{zu}$ nennen. $\mathrm{V}_{\mathrm{N}}$ ist ein Donator und hat für Fermienergien nahe der Valenzbandkante (p-Typ) und mittig in der Bandlücke (semi-isolierend) die niedrigste Bildungsenergie unter den intrinsischen Defekten.

Antisites $\left(\mathrm{Ga}_{\mathrm{N}}, \mathrm{N}_{\mathrm{Ga}}\right)$ und Zwischengitteratome $\left(\mathrm{Ga}_{\mathrm{i}}, \mathrm{N}_{\mathrm{i}}\right)$ haben in $\mathrm{GaN}$ hohe Bildungsenergien und treten damit nur in vernachlässigbaren Konzentrationen auf. Dieses ist durch den großen Unterschied der kovalenten Radien von Ga und N begründet [38]. Nur für Fermienergien sehr nahe der Valenzbandkante sind relevante Konzentrationen von $\mathrm{Ga}_{\mathrm{N}}$ bzw. $\mathrm{Ga}_{\mathrm{i}}$ zu erwarten.

Der gängigste extrinsische Donator für die Herstellung von hoch n-dotiertem GaN ist Silizium auf einem Galliumplatz $\left(\mathrm{Si}_{\mathrm{Ga}}\right)$. Seine Bildungsenergie liegt durchgängig noch knapp $1 \mathrm{eV}$ unterhalb der des $\mathrm{O}_{\mathrm{N}}$. Dies hat zur Folge, daß so der Einbau von Sauerstoff in GaN:Si unterdrückt wird [43].

P-leitendes GaN ist schwierig herzustellen. Der gängigste Akzeptor dafür ist Magnesium. Seine Aktivierungsenergie liegt bei ca. $200 \mathrm{meV}$ und ist damit eine tiefe Störstelle. Ein weiterer Akzeptor ist Zink, mit einer Aktivierungsenergie von ca. $400 \mathrm{meV}$. Flache Akzeptoren sind derzeit nicht bekannt. Aus diesem Grund ist die Effizienz der Akzeptoren in GaN gering. Ein weiterer Akzeptor in GaN ist Kohlenstoff auf einem Stickstoffplatz $\left(\mathrm{C}_{\mathrm{N}}\right)$. Das Verhalten von Kohlenstoff in GaN ist sehr komplex und nicht vollständig geklärt; es ist von Selbstkompensation durch Einbau auf verschiedenen Gitterplätzen geprägt [45]. 


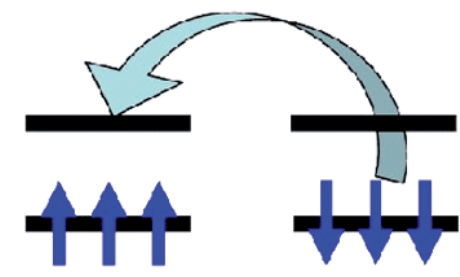

Neutral cation Vacancies

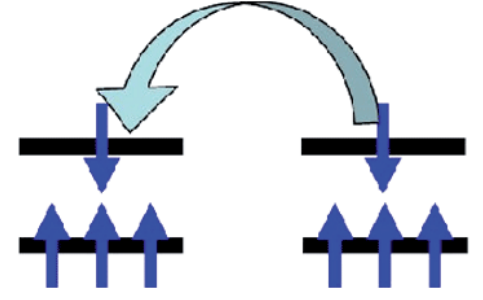

Charged cation Vacancies

Abbildung 1.5:

Skizze der Austauschwechselwirkung in $\mathrm{GaN}: \mathrm{V}_{\mathrm{Ga}}$ nach Dev et al. Im neutralen Ladungszustand ist die Wechselwirkung der Galliumleerstellen in GaN antiferromagnetisch. Werden Elektronen hinzugefügt und dadurch der Akzeptor kompensiert, so wird die Kopplung ferromagnetisch [46].

\subsection{Die Rolle der Galliumleerstelle}

Im ungeladenen Zustand trägt die Galliumleerstelle drei ungepaarte Löcher und ein Moment von $3 \mu_{\mathrm{B}}$. Energieberechnungen dieser Spinpolarisation von Dev et al. zeigen, daß dieses Moment bis deutlich über Raumtemperatur stabil sein sollte [46]. Weiter zeigen sie, daß die ungeladenen Galliumleerstellen ohne die Anwesenheit von Gd untereinander antiferromagnetisch koppeln. Der Austausch findet dabei direkt durch den Überlap der ausgedehnten Ausläufer der Defektwellenfunktionen der Leerstellen statt. Durch Kompensation, also hier das Hinzufügen von Elektronen, wird der ferromagnetische Zustand energetisch günstig (Abbildung 1.5). In diesem Szenario wird der ferromagnetische Zustand alleine durch die Anwesenheit von kompensierten Galliumleerstellen stabilisiert. Dev et al. zeigen auch in einer späteren Arbeit, daß diese Art von magnetischer Wechselwirkung zwischen kationischen Störstellen auch auf Kalium dotiertes GaN und auf $\mathrm{ZnO}$ anzuwenden ist [47].

Die ferromagnetische Wechselwirkung der Galliumleerstellen in sonst undotierem GaN wird von Gohda und Oshiyama theoretisch bestätigt [35]. Die Autoren merken aber an, daß je nach Position der Leerstellen im Kristall zueinander der ferromagnetische oder antiferromagnetische Grundzustand bevorzugt werden kann. Weiter wurde der Einfluß der Gd-Dotierung auf das GaN: $\mathrm{V}_{\mathrm{Ga}}$ System untersucht. Gd koppelt ferromagnetisch mit der Galliumleerstelle und verstärkt so die magnetischen Momente gegenüber dem undotierten GaN: $\mathrm{V}_{\mathrm{Ga}}$. Die Wechselwirkung der Gd untereinander ohne die Anwesenheit von Galliumleerstellen ist für Abstände größer $1 \mathrm{~nm}$ vernachlässigbar. Damit wird also auch das Bild vom paramagnetischen Verhalten von GaN:Gd ohne die Anwesenheit von $\mathrm{V}_{\mathrm{Ga}}$ wie bei Liu et al. bestätigt [37]. Die kolossalen magnetischen Momente werden von Gohda und Oshiyama durch die Akkumulation von $V_{\mathrm{Ga}}$ erklärt. Jede $\mathrm{V}_{\mathrm{Ga}}$ trägt im ungeladenen Zustand $3 \mu_{\mathrm{B}}$ zum effektiven Moment pro Gd Atom bei. Bis zu einer 
Anzahl von 71 Leerstellen pro Gd $\left(220 \mu_{\mathrm{B}} / \mathrm{Gd}\right)$ wurde diese Theorie überprüft und für richtig befunden.

Ein Hinweis gegen die Galliumleerstelle ist eine Arbeit von Oila et al. [43]. Es wird die Auswirkung von Dotierungen auf die Bildung der Galliumleerstelle in GaN untersucht. Eine Aussage dabei ist, daß die Galliumleerstelle nur als Komplex mit einem Sauerstoff auftritt und durch eine Dotierung mit Si effektiv unterdrückt werden kann, da hierdurch die Sauerstoffkonzentration in GaN herabgesetzt wird. Die Arbeiten von Hite et al. und Zhou etal. zeigen aber, daß auch mit einer Si-co-Dotierung GaN:Gd ferromagnetisch wechselwirkt und diese Magnetisierung sogar verstärkt werden kann [18, 23].

Schaut man sich in Abbildung 1.4 die Bildungsenergie der Galliumleerstelle oder den entsprechenden Komplex mit einem Sauerstoff an, erkennt man, daß für eine Lage der Fermienergie ungefähr mittig in der Bandlücke (semi-isolierend) bis hin zum Leitungsband (n-leitend) diese im dreifach, bzw. doppelt geladenen Zustand vorliegen. Diese Konfiguration trägt kein magnetisches Moment, da alle Löcher des Akzeptors mit Elektronen kompensiert sind, und kann so nicht zur Magnetisierung von GaN:Gd beitragen.

Auch die Arbeit von Martínez-Criado et al. [21] gibt einen Hinweis gegen die Galliumleerstelle. EXAFS Messungen an der Ga Kante zeigen keine Änderungen der Bindungslängen in der direkten Umgebung von Ga. Eine hohe Konzentration von $\mathrm{V}_{\mathrm{Ga}}$ würde aber gerade dies bewirken.

Bei der Erklärung der kolossalen Momente mit Hilfe der $\mathrm{V}_{\mathrm{Ga}}$ entsteht ein Widerspruch, wenn die Gleichgewichtskonzentration von $\mathrm{V}_{\mathrm{Ga}}$ bei Wachstumsbedingungen mit der Abschätzung aus Abschnitt 1.2.2 verglichen wird. Eine Abschätzung der Gleichgewichtskonzentration $c_{\text {def }}$ der $\mathrm{V}_{\mathrm{Ga}}$ ist mit Hilfe von Formel 1.2 möglich [38].

$$
c_{\text {def }}=N_{\text {sites }} \cdot e^{-\frac{E_{f}}{k_{B} T}}
$$

$N_{\text {sites }}$ ist dabei die Teilchendichte des Ga-Untergitter von $4,424 \cdot 10^{22} \mathrm{~cm}^{-3}, E_{\mathrm{f}}$ die Bildungsenergie und $k_{\mathrm{B}}$ und $T$ die Boltzmannkonstante und die Temperatur in K. Abbildung 1.6 zeigt die Gleichgewichtskonzentration $c_{\text {def }}$ in Abhängigkeit der Bildungsenergie für ausgesuchte Temperaturen. Die mittleren drei Temperaturen stellen typische Substrattemperaturen während des MBE-Wachstums dar, $760{ }^{\circ} \mathrm{C}$ ist die in dieser $\mathrm{Ar}$ beit verwendete Substrattemperatur. Die höhere und niedrigere Temperatur verdeutlichen den Trend, wobei ca. $1000^{\circ} \mathrm{C}$ auch eine typische Substrattemperatur während des MOCVD-Wachstums von GaN ist. Die in Abschnitt 1.2.2 abgeschätzte Konzentration der Defekte, welche die kolossalen Momente tragen könnten ist ca. $10^{19} \mathrm{~cm}^{-3}$. Eine solch hohe Gleichgewichtskonzentration wird nur unterhalb einer Bildungsenergie von $0,75 \mathrm{eV}$ erreicht. Vergleicht man diesen Wert mit den Bildungsenergien für die Galliumleerstelle in Abbildung 1.4, so findet man nur eine genügend niedrige Bildungsenergie bei einer Lage der Fermienergie direkt an der Leitungsbandkante. Diese Tatsache ist aber im 


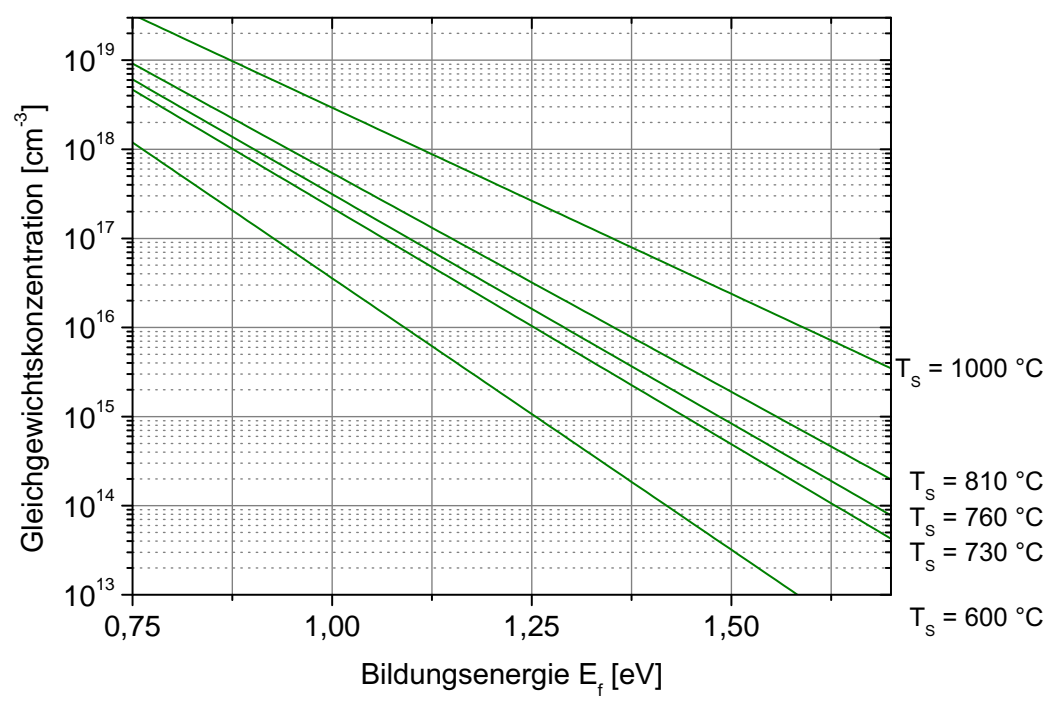

\begin{abstract}
Abbildung 1.6:
Gleichgewichtskonzentration der Galliumleerstelle $V_{G a}$ nach Formel 1.2. Die verschiedenen Temperaturen stellen typische Substrattemperaturen des GaN-Wachstums dar. Eine hohe Leerstellenkonzentration von $10^{19} \mathrm{~cm}^{-3}$ wird nur für Bildungsenergien von $0,75 \mathrm{eV}$ und weniger erreicht.
\end{abstract}

Widerspruch mit der gefundenen niedrigen Leitfähigkeit in GaN:Gd. Eine Möglichkeit diesen Widerspruch zu lösen wäre der Gedanke, daß die Galliumleerstelle als Relaxationsmechanismus für durch Gd-induzierte Verspannungen der GaN-Matrix auftreten. Es ist allerdings nicht klar warum das einbringen von Gd die GaN-Matrix unverspannt bleiben sollte, insbesondere, da es in der Literatur [26] und für die Proben dieser Arbeit (Abschnitt 5.4.3 und [48]) Anzeichen für eine verspannte Schicht gibt.

Auch wenn die genannten Hinweise noch keinen Ausschluß der Galliumleerstelle zulassen, zeigen sie doch deutlich, daß auch die Galliumleerstelle alleine nicht die experimentellen Ergebnisse zu erklären vermag. Im Rahmen dieser Arbeit konnten Positronenvernichtungsmessungen (PAS) in Kooperation mit der Aalto Universität Helsinki zeigen, daß gerade in stark ferromagnetischen Schichten mit hohen Werten der effektiven Magnetisierung $p_{\text {eff }}$ (kolossale Momente) keine $\mathrm{V}_{\mathrm{Ga}}$ gefunden wurden (Abschnitt 5.3).

\title{
1.7 Intrinsische Defekte und Sauerstoff
}

Die magnetischen Eigenschaften der gängigsten intrinsischen Defekte in GaN werden von Mitra und Lambrecht diskutiert [49]. Sie werden auf ungesättigte Bindungen des Stickstoffes in der direkten Umgebung eines Defektes zurückgeführt. Damit können $V_{N}$, $\mathrm{Ga}_{\mathrm{i}}$ oder $\mathrm{Ga}_{\mathrm{N}}$ ausgeschlossen werden. $\mathrm{V}_{\mathrm{Ga}}$ und $\mathrm{N}_{\mathrm{i}}$ erfüllen diese Bedingung. Für $\mathrm{V}_{\mathrm{Ga}}$ 
wird argumentiert, daß sie für eine Fermienergie mittig in der Bandlücke eine deutlich größere Bildungsenergie besitzt als z.B. $\mathrm{N}_{\mathrm{i}}$. Auch die Tatsache, daß die Leerstelle im dreifach geladenen Zustand nicht zur Magnetisierung beitragen kann wird angesprochen (vergl. Abbildung 1.5). $\mathrm{N}_{\mathrm{i}}$ leidet nicht an dieser Problematik. Für $\mathrm{N}_{\mathrm{i}}$ und $\mathrm{O}_{\mathrm{i}}$ wird gezeigt, daß diese auch ein magnetisches Moment aufweisen und beide jeweils untereinander ferromagnetisch koppeln. Beiden Defekten wird zugesprochen, daß sie energetisch günstiger sind als $\mathrm{V}_{\mathrm{Ga}}$ und somit wahrscheinlichere Kandidaten für das ferromagnetische Verhalten in GaN:Gd sind.

In Abbildung 1.4 wird deutlich, daß zwar $\mathrm{N}_{\mathrm{i}}$ sowie $\mathrm{O}_{\mathrm{i}}$ eine geringere Bildungsenergie haben als die $\mathrm{V}_{\mathrm{Ga}}$, die Zwischengitteratome aber nicht die jeweiligen Defekte von Stickstoff bzw. Sauerstoff mit der niedrigsten Bildungsenergie sind. Für Stickstoff gilt, daß $V_{N}$ immer eine um 2-4 eV geringere Bildungsenergie hat und damit der bevorzugte Defekt ist. Auch $\mathrm{O}_{\mathrm{N}}$ hat in großen Teilen der Lage der Fermienergie eine geringere Bildungsenergie als das zugehörige Zwischengitteratom. Lediglich für Fermienergien oberhalb von ca. $3,2 \mathrm{eV}$ wird $\mathrm{O}_{\mathrm{i}}$ gegenüber $\mathrm{O}_{\mathrm{N}}$ bevorzugt. In diesem Bereich ist aber auch der Komplex $\mathrm{V}_{\mathrm{Ga}}-\mathrm{O}_{\mathrm{N}}$ energetisch günstiger als $\mathrm{O}_{\mathrm{i}}$. Über diese Problematik hinaus leidet $\mathrm{O}_{\mathrm{i}}$ an den gleichen Ungereimtheiten wie $\mathrm{V}_{\mathrm{Ga}}$. Der über einen weiten Bereich der Bandlücke bevorzugte Ladungszustand ist zweifach geladen und damit ein Zustand ohne magnetisches Moment (Abbildung 1.4).

Das Thema der kolossalen Momente wird von Mitra und Lambrecht nur kurz angeschnitten. Es wird festgestellt, daß noch keine Gründe für solch hohen Defektkonzentrationen gefunden wurden, um kolossale Momente zu erklären. Geht man allerdings von dem von Mitra und Lambrecht vorgeschlagenen $\mathrm{O}_{\mathrm{i}}$ aus, so ist bei einer Gd Konzentration von $10^{15} \mathrm{~cm}^{-3}$ und einem effektiven Moment von $4000 \mu_{\mathrm{B}} / \mathrm{Gd}$ eine Konzentration von $\mathrm{O}_{\mathrm{i}}$ von $2 \cdot 10^{18} \mathrm{~cm}^{-3}$ notwendig. ${ }^{1}$ Eine solche Konzentration ist nicht ungewöhnlich und wurde z.B. von Hite et al. berichtet $\left(10^{19} \mathrm{~cm}^{-3}\right.$ [31]).

\section{Zusammenfassung}

Experimentelle und theoretische Arbeiten über GaN:Gd zeigen eine große Bandbreite an Fakten und Modellen, welche den Ferromagnetismus beschreiben. GaN:Gd ist ferromagnetisch bei Raumtemperatur. Die effektiven Momente pro Gd-Atom zeigen bei Dhar et al. Werte bis zu $10^{5} \mu_{\mathrm{B}}$. Diese sind bis zu diesem Zeitpunkt weder mit theoretischen Modellen, noch mit experimentellen Arbeiten zu erklären. Schon kleine Mengen an Gd führen zu hochohmigen Verhalten der Schichten und die elektrischen Eigenschaften sind von VRH-Leitfägikeit in einem schmalen Defektband geprägt. Theoretische Arbeiten sind widersprüchlich und sagen die Stabilisierung der ferromagnetischen Phase über

\footnotetext{
${ }^{1}$ Das Sauerstoff Zwischengitteratom trägt $2 \mu_{\mathrm{B}}$ im neutralen Ladungszustand.
} 
Elektronen bzw. Löcher im System voraus. Auch Defekte werden im Zusammenhang mit dem Magnetismus diskutiert. Besonders ist hier die Galliumleerstelle zu nennen.

Auch für GaN:Gd stellt sich wie bei anderen DMS-Systemen die Frage, ob die magnetischen Eigenschaften auf eine verdünnte Phase oder auf Ausscheidungen zurückzuführen sind und ob Defekte eine Rolle bei der magnetischen Wechselwirkung spielen. Dhar et al. beobachten einen magnetischen Übergang bei ca. $70 \mathrm{~K}$, welcher auf GdN Ausscheidungen schließen läßt. Diese Ausscheidungen können aber weder experimentell bestätigt werden, noch können sie den Ferromagnetismus bei $300 \mathrm{~K}$ erklären. Die Bildung von nanoskaligen Gd-Ausscheidungen oder spinodale Entmischung kann zu diesem Zeitpunkt nicht ausgeschlossen werden.

Die Frage ob GaN:Gd ein verdünnter magnetischer Halbleiter ist kann somit zu diesem Zeitpunkt nicht beantwortet werden. Sicher ist, daß die beobachteten Phänomene nicht durch die Gd-Dotierung alleine erklärt werden können und Parameter wie Defekte oder Dotierungen eine Rolle zu spielen scheinen. Die folgenden Kapitel gehen anhand einer Analyse von Zusammenhängen zwischen strukturellen und magnetischen Eigenschaften auf diese Frage ein und tragen so zum weiteren Verständnis des Systems GaN:Gd bei. 


\title{
Kapitel 2
}

\section{Herstellung von Gadolinium dotiertem GaN}

\begin{abstract}
Alle Schichten dieser Arbeit wurden mittels MBE in der Anlage ELSA hergestellt. Diese wird kurz in Abschnitt 2.1 vorgestellt. Die Substratwahl und die Vorbereitung dieses werden in den Abschnitten 2.2 und 2.3 beschrieben. Insgesamt wurden ca. 80 GaN:GdSchichten mit verschiedenen co-Dotierungen und unterschiedlichen Wachstumsparametern hergestellt und charakterisiert. Die zugrundeliegende Systematik bei der Herstellung der verschiedenen GaN:Gd-Schichten und die Wahl der Wachstumsparameter sind in Abschnitt 2.4 zusammengefaßt. Abschließend wird in Abschnitt 2.5 der Einfluß des Gd auf die Wachstumsrate des GaN untersucht.
\end{abstract}




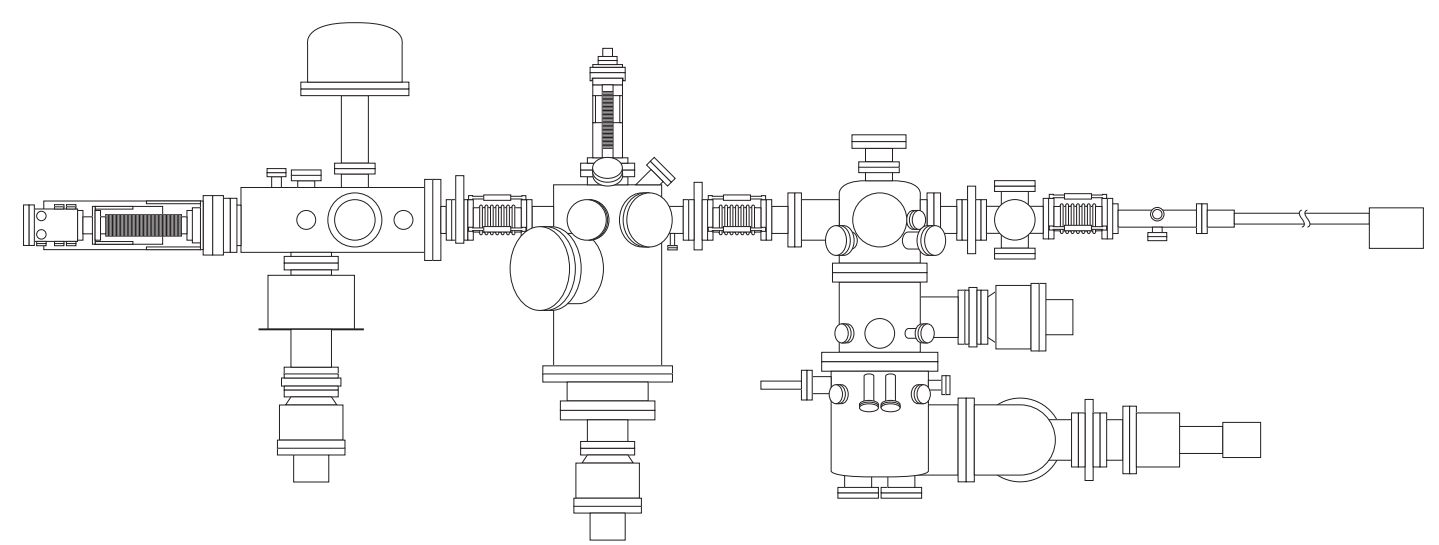

Abbildung 2.1:

Skizze der Molekularstrahlepitaxie Anlage ELSA. Kammern von Links nach rechts: Röntgenspektroskopie (XPS), Oberflächenanalytik mit AES und LEED, Wachstum, Schleuse und Transfer.

\subsection{ELSA - Epitaxie LEED und Spektroskopie Anlage}

ELSA besteht insgesamt aus drei Kammern und einem Schleusen- und Transfersystem (Abbildung 2.1). Von Links nach Rechts gesehen sind die Kammern wie folgt eingerichtet: Röntgenspektroskopie, Oberflächenanalytik und Wachstumskammer. An die Wachstumskammer schließen sich Schleuse und Transfer an. Die einzelnen Kammern werden im folgenden kurz vorgestellt.

Röntgenspektroskopie (XPS) In der hinteren Kammer (von der Schleuse aus gesehen) ist die Röntgenspektroskopie ( $x$-ray photon spectroscopy - XPS) installiert. Sie wird mit einer Turbomolekularpumpe auf einem Basisdruck von ca. $10^{-9}-10^{-10}$ mbar gehalten. Die direkte Verbindug mit der Wachstumskammer läßt in-situ Messungen ohne den Bruch des Vakuums zu. Dies ist besonders für XPS-Messungen attraktiv, da die Methode mit ca. $1 \mathrm{~nm}$ Eindringtiefe sehr Oberflächensensitiv ist. Die Anregung wird durch eine $15 \mathrm{kV}$ Röntgenröhre und einen Siliziummonochromator realisiert. Diese Kombination läßt eine monochromatische Anregung mit der Al $\mathrm{K}_{\alpha}$ Linie zu. Eine Elektronenoptik und ein Kugelanalysator gefolgt von zwei Multichannelplates werten die in der Probe ausgelösten Elektronen aus und erreichen eine Energieauflösung von ca. $50 \mathrm{meV}$.

Um auch ex-situ Proben mit XPS charakterisieren zu können steht eine einfache Ar-Sputterkanone der Firma Specs zur Verfügung. Es kann mit einer maximalen Beschleunigungsspannung von $3 \mathrm{kV}$ gearbeitet werden, um die Oberfläche effektiv von Verunreinigungen zu befreien. 
Oberflächenanalytik - AES und LEED Die mittlere Kammer beherbergt AES (auger electron spectroscopy) und LEED (low energy electron diffraction). Sie wird durch eine Turbomolekularpumpe auf einem Basisdruck von ca. $10^{-9}-10^{-10}$ mbar gehalten.

AES ist eine Spektroskopiemethode, bei der der Augerprozess genutzt wird, um Aussagen über Materialzusammensetzung und die atomare Umgebung einzelner Komponenten zu machen. Bindungsenergien und Konzentrationen der einzelnen Komponenten können bestimmt werden. Die Eindringtiefe dieser Methode beschränkt sich auf wenige Nanometer. Sie ist besonders geeignet um Oberlächenkontaminationen zu erkennen und so die Substratvorbehandlungen zu optimieren.

LEED ist eine Beugungsmethode, bei der ein senkrecht zur Probenoberfläche einfallender Elektronenstrahl gebeugt wird. Das entstehende Beugungsmuster läßt Rückschlüsse auf Gitterparameter und Orientierung zu. Überstrukturen auf der Oberfläche spiegeln sich im Beugungsbild in sog. Rekonstruktionen wider. Anhand dieser lassen sich unteranderem Rückschlüsse auf Verunreinigungen an der Oberfläche ziehen.

Wachstumskammer Die Wachstumskammer besteht aus einem Rezipienten, an den sich direkt die Oberflächenanalyse zur einen und die Schleusen- und Transfereinheit zur anderen Seite anschliefst. Sie wird durch eine Turbomolekularpumpe, eine Kryopumpe und eine Titangetterpumpe gepumt und so auf einem Basisdruck von ca. $10^{-10}$ $10^{-11}$ mbar gehalten. Zwei Flüssigstickstofftanks entkoppeln die Materialquellen thermisch voneinander und dienen als Kühlfalle für die Titangetterpumpe.

Im unteren Bereich der Wachstumskammer sind vier Flansche für die Aufnahme von Materialquellen in einem Winkel von $33^{\circ}$ auf die Wachstumsposition fokussiert. Drei der Aufnahmen beherbergen Effusionszellen, die mit den Elementen Gallium (7N), Gadolinium $(4 \mathrm{~N})$ und Silizium $(6 \mathrm{~N})$ gefüllt sind. In der vierten Aufnahme befindet sich eine RF-Plasmaquelle, Model Unibulb der Firma EPI für die Bereitstellung von aktiviertem und atomarem Stickstoff.

In den Fokus der Materialquellen kann ein Probenhalter gebracht werden, der einen Heizer enthält. Es können hier Wachstumstemperaturen von bis zu $1000^{\circ} \mathrm{C}$ erreicht werden. Der Heizer ist direkt im mobilen Teil des Probenhalters montiert und kann so mit der Probe in jede Kammer der Anlage transferiert werden.

\subsection{Substratwahl und -temperatur}

Für die hergestellten Schichten dienen mit MOCVD hergestellte GaN Schichten auf Saphir. Das MOCVD-GaN hat eine Dicke von ca. $2 \mu \mathrm{m}$ und wird direkt, also ohne weitere Pufferschichten als Substrat verwendet. Die endgültige Probenstruktur ist in Abbildung 2.2(a) skizziert. 


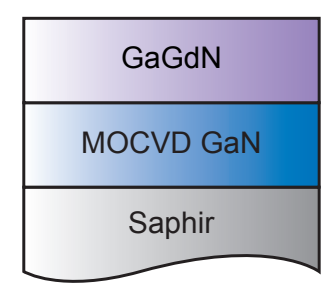

(a) MOCVD GaN

\begin{tabular}{ccc}
\hline \hline Reinigung & Aceton & $2 \mathrm{~min}$ \\
& $\mathrm{H}_{2} \mathrm{O}$ & $2 \mathrm{~min}$ \\
& Aceton & $2 \mathrm{~min}$ \\
& Propanol & $2 \mathrm{~min}$ \\
\hline im UHV & $\mathrm{T}_{\mathrm{S}}=550^{\circ} \mathrm{C}$ & $1 \mathrm{~h}$ \\
& $\mathrm{~T}_{\mathrm{S}}=760^{\circ} \mathrm{C}$ & \\
& Nitridierung & $10 \mathrm{~min}$ \\
\hline
\end{tabular}

(b) Vorbehandlung

\section{Abbildung 2.2:}

(a) Skizze der verwendeten Probenstruktur. Die GaN:Gd Schichten wurden direkt auf $2 \mu \mathrm{m}$ MOCVD GaN hergestellt und haben i.d.R. eine Dicke von $500 \mathrm{~nm}$. Es wurden keine Pufferschichten zwischen Template und Schicht eingeführt. (b) Zusammenfassung der Schritte der Vorbehandlung der Substrate.

Der große Vorteil von homoepitaktischem Wachstum, also dem Wachstum auf Gleichem ist der Ausschluß von Einflüssen durch thermische Fehlpassung und Gitterfehlpassung auf das Schichtwachstum. Für die Homoepitaxie von GaN sind sog. free standing GaN Schichten als Substrat nötig. Diese sind erst seit kurzer Zeit kommerziell erhältlich und teuer. Die wirtschaftlichste Näherung zu Volumen-GaN sind dicke MOCVD-GaNSchichten auf Saphir. Der Einfluß des unterliegenden Saphir Substrates kann bei Schichtdicken von $2 \mu \mathrm{m}$ verringert werden und kommt damit der Homoepitaxie auf VolumenGaN sehr nahe. Allerdings sind Verspannungen durch das unterliegende Saphir-Substrat selbst bei $80 \mu \mathrm{m}$ dicken GaN-Schichten nicht komplett zu eliminieren [50].

Die absolute Messung der Oberflächentemperatur von GaN in der MBE ist mit einigen Problemen behaftet. Üblicherweise wird die Temperatur der Oberfläche mit Hilfe von Pyrometern bestimmt. Saphir und GaN sind aber im Wellenlängenbereich der meisten handelsüblichen Pyrometer durchsichtig und es wird zusätzlich zu dem Signal von der GaN-Schicht auch der unterliegende Heizer direkt gemessen. Es sind spezielle Lösungen wie in den Arbeiten von Creighton etal. nötig um GaN auf Saphir direkt zu messen $[51,52]$.

Da eine solche Lösung nicht zur Verfügung stand, wurde versucht die Substrattemperatur möglichst gleichmäßig zu halten, indem der durch den Heizer fließende Strom konstant gehalten wurde. Eine Temperaturkalibrierung des verwendeten Probenhalters findet sich in Anhang D. Im folgenden wird immer $\mathrm{T}_{\text {set }}$ angegeben, welche bei den verwendeten MOCVD-GaN-Templates ca. $45^{\circ} \mathrm{C}$ oberhalb $\mathrm{T}_{\text {absolut }}$ liegt. 
Tabelle 2.1: Übersicht Wachstumsparameter

\begin{tabular}{lllll}
\hline \hline & & & erste Serie & zweite Serie \\
\hline Bedingungen & Substrattemperatur & $\mathrm{T}_{\mathrm{S}}$ & $760^{\circ} \mathrm{C}$ & $760{ }^{\circ} \mathrm{C}$ \\
für optimiertes & Stickstofffluß & $\mathrm{F}_{\mathrm{N}}$ & $1,17 \mathrm{sccm}$ & $1,5 \mathrm{sccm}$ \\
GaN Wachstum: & Plasmaquellenleistung & $\mathrm{P}_{\mathrm{N}}$ & $450 \mathrm{~W}$ & $450 \mathrm{~W}$ \\
& Ga Fluß & $\mathrm{BEP}_{\mathrm{Ga}}$ & $5,5 \cdot 10^{-8} \mathrm{mbar}$ & $9 \cdot 10^{-8} \mathrm{mbar}$ \\
\hline Varierte & Substrattemperatur & $\mathrm{T}_{\mathrm{S}}$ & optimiert & $650-780^{\circ} \mathrm{C}$ \\
Prameter: & Stickstofffluß & $\mathrm{F}_{\mathrm{N}}$ & optimiert & $1-2,5 \mathrm{sccm}$ \\
& Plasmaquellenleistung & $\mathrm{P}_{\mathrm{N}}$ & optimiert & $160 \mathrm{~W}$ oder $450 \mathrm{~W}$ \\
& Ga Fluß & $\mathrm{BEP}_{\mathrm{Ga}}$ & optimiert & $2,5-20 \cdot 10^{-8} \mathrm{mbar}$ \\
& Gd Quellentemperatur & $\mathrm{T}_{\mathrm{Gd}}$ & $675-140{ }^{\circ} \mathrm{C}$ & $800{ }^{\circ} \mathrm{Coder} 1100^{\circ} \mathrm{C}$ \\
& Si Quellentemperatur & $\mathrm{T}_{\mathrm{Si}}$ & $1400^{\circ} \mathrm{C}$ & - \\
& Wasserstofffluß & $\mathrm{F}_{\mathrm{H}}$ & $0,1 \mathrm{sccm}$ & - \\
& Wasserstoffquellenleistung & $\mathrm{P}_{\mathrm{H}}$ & $180 \mathrm{~W}$ & - \\
\hline
\end{tabular}

\subsection{Vorbehandlung}

Die Substrate wurden vor dem Einbau ins UHV mit Lösungsmitteln gereinigt und unverzüglich in die Anlage eingebracht. Vor dem Wachstum der Schicht wird das Substrat bei $550^{\circ} \mathrm{C}$ ausgeheizt, um Wasser und natürliche Oxide von der Oberfläche zu entfernen. Die Dauer des Ausheizvorganges richtet sich nach dem Kammerdruck und dauert ca. 1 Stunde. Ist der Druck stabil so wird die Wachstumstemperatur angefahren. Zu Begin wird die Substratoberfläche für 10 min dem aktivierten Stickstoff ausgesetzt. Direkt im Anschluß an diese Nitridierung wird die Herstellung der Schicht begonnen. Nach dem Wachstum der Schicht folgt eine weitere Nitridierung der Oberfläche, um überschüssiges Ga zu GaN zu wandeln. Die Details sind in der Tabelle in Abbildung 2.2(b) zusammengefaßst.

\subsection{Herstellung von GaN:Gd mit MBE}

Grob sind die hergestellten Schichen in zwei verschiedene Serien einzuteilen. In einer ersten Serie wurde die Dotierung von GaN mit Gd und der Einfluß von co-Dotierungen untersucht. Die zweite Serie untersucht die Einflüsse der verschiedenen Wachstumsparameter, wie Substrattemperatur $\mathrm{T}_{\mathrm{S}}$ oder das III-V-Verhältnis. Diese Aufteilung der Serien ist vorwiegend aus Problemen bei der Reproduzierbarkeit der magnetischen Eigenschaften entstanden. Obwohl bei beiden Serien die strukturellen Eigenschaften scheinbar reproduzierbar sind (Abschnitt 5.1), zeigen sich große Unterschiede in den magentischen Eigenschaften. Dieses deutet auf den Einfluß eines nicht kontrollierten Parameters hin und soll durch die Variation der Wachstumsparameter in der zweiten Serie untersucht 
werden. Vorausgenommen sei an dieser Stelle, daß die Variation der Wachstumsparameter keine Einsicht gebracht hat, auf welchen Parameter die magnetischen Eigenschaften sensitiv sind.

Tabelle 2.1 faßst die Variationen der verschiedenen Parameter in den zwei Serien zusammen. Die Wahl der Parameter für das optimierte GaN-Wachstum der Serie 1 basiert auf früheren Ergebnissen der Arbeitsgruppe, in denen unter anderem das Wachstum von nicht dotiertem GaN optimiert wurde. Für das Wachstum der Gd-dotierten GaNSchichten wurden die optimierten Parameter verwendet und konstant gehalten. Die GdDotierung wurde mit variierenden Gd-Zellentemperaturen erreicht und ist im Bereich von ca. $10^{14}$ bis $10^{20} \mathrm{~cm}^{-3}$ angesiedelt (Kapitel 3). Als co-Dotierungen wurden Silizium, Wasserstoff und Sauerstoff untersucht. Es wurden die selben Parameter verwendet wie bei den nicht co-dotierten GaN-Schichten. Für die co-Dotierung mit Si wurde eine Si Dotierquelle verwendet, deren Temperatur $1400^{\circ} \mathrm{C}$ betrug. Die $\mathrm{H}$ co-Dotierung wurde mit Hilfe einer Quelle für aktivierten Wasserstoff vorgenommen, wie sie in [53] beschrieben ist. Dabei wurde ein $\mathrm{H}_{2}$ Fluß von $0,1 \mathrm{sccm}$ und eine Leistung von $180 \mathrm{~W}$ verwendet. Sauerstoff wurde als nicht aktiviertes Gas mit unterschiedlichen Flüssen in die Kammer eingelassen, um so verschiedene Sauerstoffhintergrunddrücke zu erzeugen. Da für diese Materialien keine Standards für die absolute Bestimmung der Dotierungen zur Verfügung stehen, werden im folgenden diese Parameter als Anhaltspunkte verwendet.

Die Serie 2 hat gegenüber der Serie 2 veränderte Parameter für das optimierte Wachstum von GaN. Dieses liegt an Wartungsarbeiten an der MBE-Anlage. Während der Wartung wurde unter anderem die Stickstoffplasmaquelle zur Generalüberholung zum Hersteller eingeschickt (Tiegelreinigung und neues Design der Lochplatte). Vor und nach diesen Veränderungen der Stickstoffplasmaquelle wurde mit Hilfe optischer Spektroskopie das Plasma charakterisiert und für das GaN Wachstum optimiert (möglichst hoher Anteil an atomarem Stickstoff, [54, 55]). So wurde sichergestellt, daß auch nach der Wartung vergleichbare Bedingungen für das Wachstum herrschen. Zur Untersuchung der Einflüsse durch die Stickstoffplasmaquelle und die Wachstumsbedingungen allgemein wurden dann in systematischer Weise die Parameter Substrattemperatur $\mathrm{T}_{\mathrm{S}}$, Stickstofffluß $\mathrm{F}_{\mathrm{N}}$, Stickstoffplasmaleistung $\mathrm{P}_{\mathrm{N}}$ und der Ga-Fluß $\mathrm{BEP}_{\mathrm{Ga}}{ }^{1}$ variiert. Da unter anderem auch Stickstoffzwischengitteratome im Verdacht stehen die magnetischen Eigenschaften in GaN:Gd zu beeinflussen [49, 23], wurden auch Versuche durchgeführt alle Änderungen an der Stickstoffplasmaquelle zurückzubauen und erneut mit den Parametern der Serie 1 GaN:Gd herzustellen.

Alle wichtigen Daten zu den hergestellten Schichten sind in Anhang A in Tabelle A.1 und A.2 zusammengestellt. Auf diese Tabelle kann im Laufe der folgenden Diskussionen immer als Übersicht zurückgegriffen werden.

\footnotetext{
${ }^{1} \mathrm{BEP}$ - beam equivalent pressure
} 


\subsection{Einfluß der Gd-Dotierung auf die Wachstumsrate}

Abbildung 2.3(a) zeigt vergleichend die Wachstumsdiagramme der beiden Serien. Es ist die Wachstumsrate der Schicht gegen den $\mathrm{BEP}_{\mathrm{Ga}}$ aufgetragen. Der gezeigte Fehler ist die Standardabweichung aus mehreren Meßwerten bei der Schichtdickenbestimmung, welche mit Hilfe eines Dektak Oberflächenprofilometers durchgeführt wurde.

Die Plasmabedingungen sind für Serie 1 und Serie 2 unterschiedlich, innerhalb der Serien aber konstant. Die erste Auffälligkeit ist der unterschiedliche Stöchiometriepunkt, welcher auf die unterschiedlichen Lochplatten der Stickstoffplasmaquelle zurückzuführen ist. Für Serie 2 sind nur Proben gezeigt, welche bei optimierten Plasmabedingungen und Substrattemperatur hergestellt wurden. Für die Wachstumsrate der um den stöchiometrischen Punkt hergestellten Schichten ist eine vergleichsweise hohe Streuung der Werte zu erkennen. Diese kann verschiedene Ursachen haben. Die Materialflüsse der Effusionsquellen können durch Änderungen im Füllgrad oder Bildung von Tropfen an der Tiegellippe schwanken. Der Hintergrunddruck der Kammer kann einen Einfluß auf den Fluß haben (vgl. Kapitel B). Eine Schwankung der Substrattemperatur zwischen den verschiedenen Proben kann eine Verlagerung des stöchiometrischen Punktes und der Wachstumsrate im metallreichen Bereich verursachen, da die Desorption der Wachstumsspezien verändert wird.

Um den Einfluß von Gd auf die Wachstumsrate zu untersuchen ist in Abbildung 2.3(b) die Wachstumsrate der beiden Serien gegen die Gd-Zellentemperatur aufgetragen. Im Rahmen des Fehlers ${ }^{2}$ ist diese unabhängig von der Gd-Zellentemperatur.

\section{Zusammmenfassung}

Alle GaN:Gd-Schichten wurden in der MBE Anlage ELSA hergestellt. Insgesamt wurden ca. 80 Schichten in zwei Serien auf MOCVD-GaN-Templates hergestellt. Die Serie 1 beschäftigt sich mit den Eigenschaften des Gd-dotierten GaN und mit co-dotierten GaN:Gd-Schichten. Es wurde mit Wasserstoff, Silizium oder Sauerstoff co-dotiert. Die Serie 2 untersucht die Auswirkungen der verschiedenen Wachstumsparameter auf die Eigenschaften des GaN:Gd.

Für beide Serien ist keine Abhängigkeit der Wachstumsrate von der Gd-Dotierung zu erkennen. Diese Daten sind in Einklang mit den von Dhar et al. und Hite et al., welche keinen Einfluß der Gd-Dotierung auf den Wachstumsmodus (2D/3D) von GaN zeigen (Abschnitt 1.1).

\footnotetext{
${ }^{2}$ hier aus Übersichtsgründen nicht eingetraten, Skala ist die gleiche wie in Abbildung 2.3(a)
} 


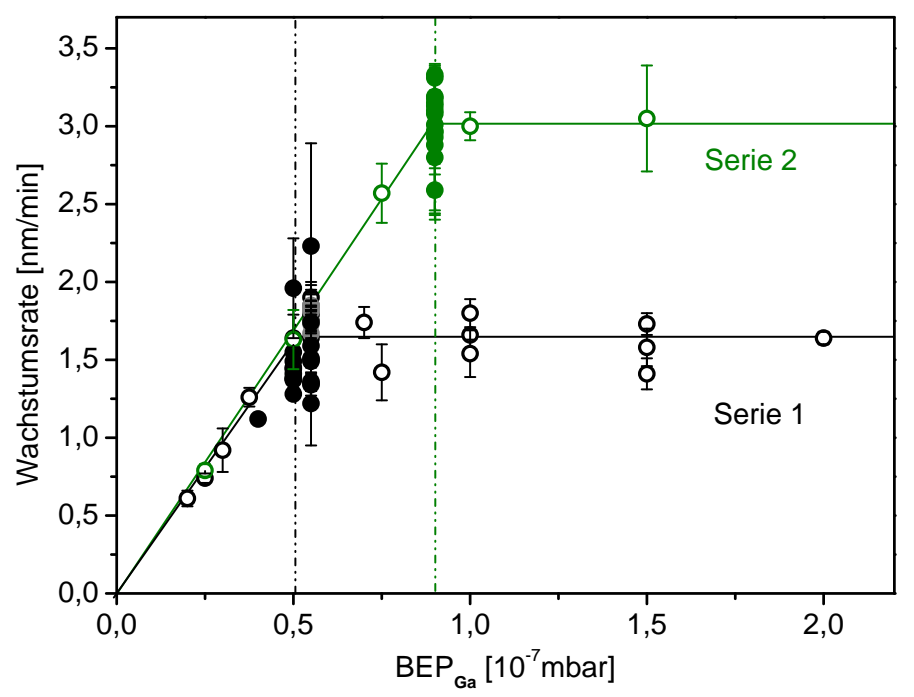

(a) Wachstumsdiagram

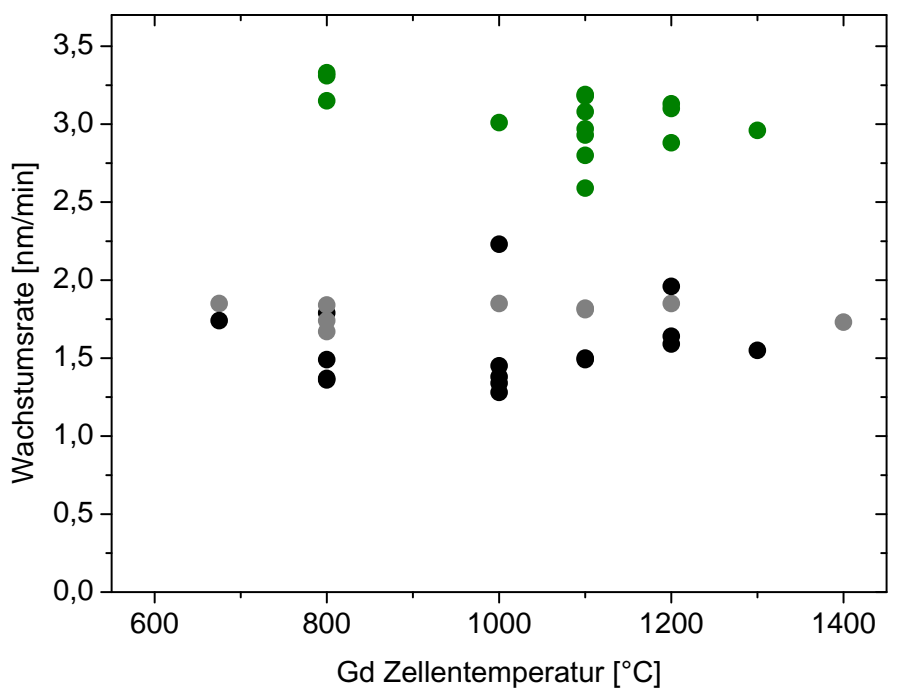

(b) Abhängigkeit der Wachstumsrate von der Gd Zellentemperatur

\section{Abbildung 2.3:}

Schwarze bzw. graue Punkte stehen für Proben der Serie 1, grüne für Proben der Serie 2. Offene Symbole zeigen nominell undotiertes GaN, geschlossene stehen für GaN:Gd. Graue Symbole sind co-dotiertes GaN:Gd. Abbildung (a) zeigt das Wachstumsdiagram, Abbildung (b) die Abhängigkeit der Wachstumsrate von der Gd Zellentemperatur. 


\section{Kapitel 3}

\section{Gadolinium Konzentration}

Ein wichtiger Aspekt dieser Arbeit war eine genaue Bestimmung der Gd-Konzentration in den GaN:Gd-Schichten. In der Literatur wurden kolossale Momente von bis zu $10^{5} \mu_{\mathrm{B}}$ pro Gd-Atom gemeldet [56, 26]. Diese Größe hat eine direkte Abhängigkeit von der GdKonzentration. Auch können andere Verunreinigung, wie z.B. Übergangsmetalle magnetische Momente tragen oder erzeugen, die Einfluß auf das experimentell bestimmte Moment pro Gd-Atom haben. Daher ist eine genaue Bestimmung der Gd-Konzentration essentiell. Die Auswertung der mit TOF-SIMS (time of flight secondary ion mass spectroscopy) gewonnenen Daten hat sich als schwierig herausgestellt. Massenspektren bzw. Tiefenprofile einzelner Massen, insbesondere die Gd-Linien, weisen Masseninterferenzen auf. Aus diesen Gründen werden in den folgenden Abschnitten die Bestimmung der Gd-Konzentration (Abschnitt 3.1 bis 3.5), eine theoretische Abschätzung der zu erwartenden Gd-Konzentration (Abschnitt 3.7) und der Aspekt von Verunreinigungen aus dem Quellenmaterial (Abschnitt 3.8) ausführlich behandelt. 


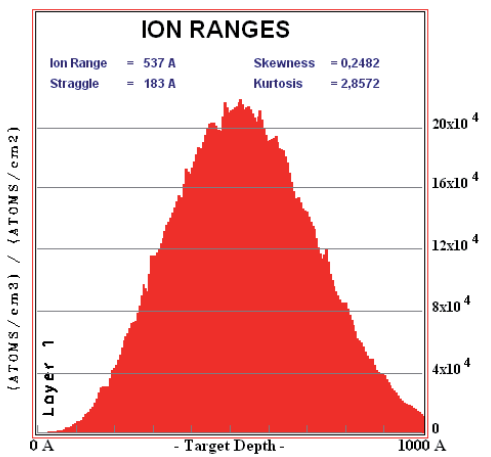

(a) TRIM Simulation

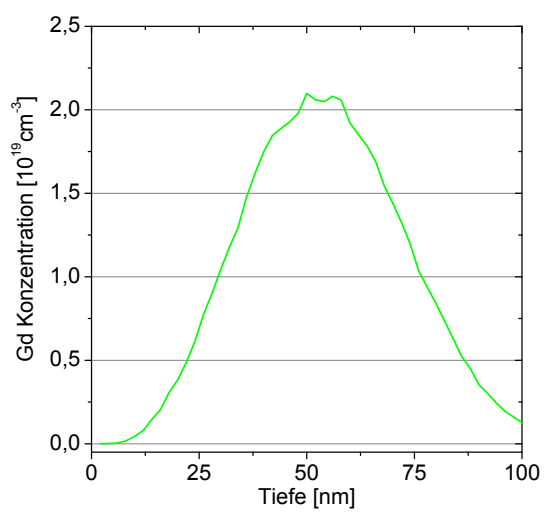

(b) Gd Konzentrationsprofil

\section{Abbildung 3.1:}

TRIM Simulation der Gd Implantation, (a) zeigt die projizierte Eindringtiefe der ${ }^{156} \mathrm{Gd}$ lonen bei einer Implantation mit $300 \mathrm{keV}$, (b) zeigt das resultierende theoretische Konzentrationsproflil bei einer Fluenz von $10^{14} \mathrm{~cm}^{-2}$.

\subsection{SIMS Standard und TRIM Simulation}

Da SIMS Messungen a priori keine absoluten Konzentrationen der enthaltenen Elemente liefern ist die Kalibrierung der Messung mit einem Standard notwendig. Typischerweise wird dazu das zu quantifizierende Element in bekannter Menge implantiert. Hier wurde in eine MBE-GaN-Schicht Gd mit folgenden Parametern implantiert.

- Masse $156 \mathrm{u}$

- Ionenenergie $300 \mathrm{keV}$

- Verkippungswinkel zur c-Achse des GaN $3^{\circ}$

- Fluenz $10^{14} \mathrm{~cm}^{-2}$

Die Wahl des ${ }^{156} \mathrm{Gd}$ als Implantationsisotop basiert dabei auf dem Isotopenverhältnis von Gd (Tabelle 3.1). Eine bessere Wahl wäre ${ }^{160} \mathrm{Gd}$, da für dieses Isotop, wie im folgenden erläutert, keine Masseninterferenzen vorliegen.

Das Implantationsprofil wurde vor der Implantation mit der Software TRIM simuliert, um die Parameter für die Implantation abzuschätzen. Abbildung 3.1(a) zeigt die projizierte Eindringtiefe der Ionen. Das Maximum liegt bei einer Tiefe von $54 \mathrm{~nm}$. Abbildung 3.1(b) zeigt das mit der Fluenz von $10^{14} \mathrm{~cm}^{-2}$ resultierende, theoretische Konzentrationsprofil der Implantation mit einer maximalen Konzentration von ca. $10^{19} \mathrm{~cm}^{-3}$.

In Abbildung 3.2(a) ist die TOF-SIMS-Messung des Gd-Standards gezeigt. Durch einen sehr weit eingestellten Massenfilter sind im Standard neben ${ }^{156} \mathrm{Gd}$ auch die Isotope 


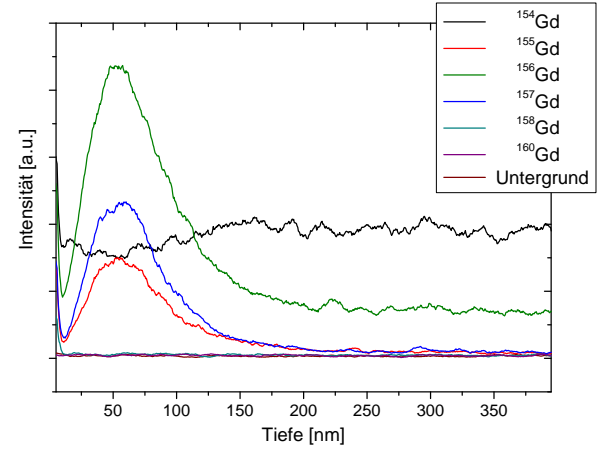

(a) Profil mit Masseninterferenzen

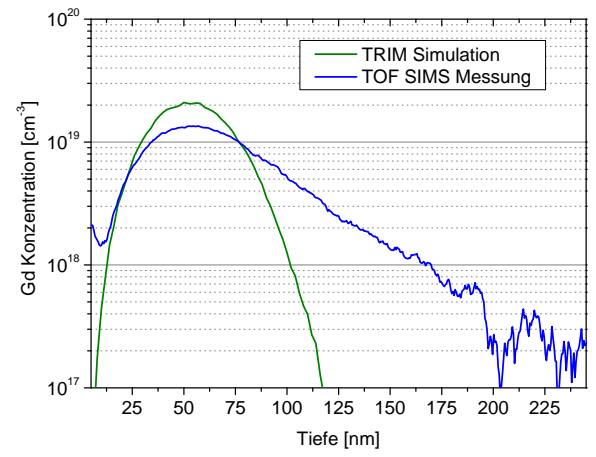

(b) Vergleich Messung - Simulation

\begin{abstract}
Abbildung 3.2:
TOF-SIMS Messung des Gd Standards, (a) Es sind die Tiefenprofile der Isotope mit einem Massenanteil von mehr als $2 \%$ gezeigt. Für ${ }^{156} \mathrm{Gd}$ ist deutlich eine Masseninterferenz zu erkennen, da das Profil für Tiefen über $200 \mathrm{~nm}$ nicht auf den Untergrund abfällt. (b) Zeigt einen Vergleich des über die Isotope integrierten Gd Profiles (nach Interferenzkorrektur) mit der TRIM Simulation aus Abbildung 3.1(b)
\end{abstract}

${ }^{155} \mathrm{Gd}$ und ${ }^{157} \mathrm{Gd}$ vertreten. Für diese Isotope ist das Implantationsprofil zu erkennen. Das Maximum liegt in guter Übereinstimmung mit der TRIM Simulation bei ca. $50 \mathrm{~nm}$.

\title{
3.2 Masseninterferenzen
}

Gd hat im natürlichen Isotopenverhältnis Anteile im Massenbereich von 152 bis $160 \mathrm{u}$. Die Isotopenverteilung ist in Tabelle 3.1 aufgelistet. Zur Erhöhung der Sensitivität wird beim TOF-SIMS unter einer Sauerstoffatmosphäre von $10^{-6}$ mbar unter sonst UHVBedingungen gemessen. Aus diesem Grund sind auch im Massenbereich 152 bis $158 \mathrm{u}$ Signale von Ga-N-Clustern und Ga-O-Clustern und deren Wasserstoffrepliken zu erwarten (vgl. Tabelle 3.1).

Am Profil von ${ }^{156} \mathrm{Gd}$ in Abbildung 3.2(a) ist deutlich eine Masseninterferenz zu erkennen, da dieses nicht auf den Untergrund abfällt. Auch ${ }^{155} \mathrm{Gd}$ und ${ }^{157} \mathrm{Gd}$ zeigen leichte Interferenzen, welche wahrscheinlich auf Wasserstoffrepliken zurückzuführen sind. Der Standard enthält damit keine Gd Isotope ohne Masseninterferenzen. Diese Tatsache ist aber insofern unproblematisch, da die Interferenzen gut identifiziert und quantifiziert werden können (Abschnitt 3.4). Weiter ist zu bemerken, daß in allen weiteren Messungen ${ }^{160} \mathrm{Gd}$ keine Interferenzen aufweist und somit das vertrauenswürdigste Signal bei der Auswertung der SIMS Daten liefert.

Auf die Masseninterferenzen ist auch die teilweise inkonsistente Angabe von Konzentrationen in den Veröffentlichungen von Röver et al. und Martínez-Criado et al. zurück- 
Tabelle 3.1: Isotopenanteile und mögliche Masseninterferenzen der Gd Isotope

\begin{tabular}{lccccccc}
\hline \hline Masse [u] & 152 & 154 & 155 & 156 & 157 & 158 & 160 \\
Isotopenanteil & $0,2 \%$ & $2,18 \%$ & $14,8 \%$ & $20,47 \%$ & $15,65 \%$ & $24,84 \%$ & $21,86 \%$ \\
J0174 & & $2,09 \%$ & $15,3 \%$ & $20,72 \%$ & $15,81 \%$ & $24,86 \%$ & $21,21 \%$ \\
Abweichung & $4,1 \%$ & $-3,4 \%$ & $-1,2 \%$ & $-1,0 \%$ & $-0,1 \%$ & $3,0 \%$ \\
\hline \multicolumn{2}{l}{ Masseninterferenzen } & & & & & & \\
Ga-N-Cluster & ${ }^{69} \mathrm{Ga}_{2}{ }^{14} \mathrm{~N}$ & ${ }^{69} \mathrm{Ga}^{71} \mathrm{Ga}^{14} \mathrm{~N}$ & $\mathrm{H}-\mathrm{Rep}$. & ${ }^{71} \mathrm{Ga}_{2}{ }^{14} \mathrm{~N}$ & $\mathrm{H}-$ Rep. & & \\
Ga-O-Cluster & & ${ }^{69} \mathrm{Ga}_{2}{ }^{16} \mathrm{O}$ & H-Rep. & ${ }^{69} \mathrm{Ga}^{71} \mathrm{Ga}^{16} \mathrm{O}$ & H-Rep. & ${ }^{71} \mathrm{Ga}_{2}{ }^{16} \mathrm{O}$ & \\
\hline
\end{tabular}

zuführen [19, 21]. Anfängliche Auswertungen der SIMS Daten haben die Interferenzen nicht berücksichtigt und so ein um ca. eine Größenordnung zu hohes Ergebnis zur Folge.

\subsection{Auflösungsvermögen und Fehler}

Das Auflösungsvermögen des TOF-SIMS für Gd wurde anhand der gemessenen Schichten mit geringer Gd Konzentration bestimmt. Das kleinste noch aufgelöste ${ }^{160} \mathrm{Gd}$ Signal entspricht einer Gd Konzentration von $2 \cdot 10^{16} \mathrm{~cm}^{-3}$ und wird als die Auflösungsgrenze für Gd in GaN angesehen.

Um den Fehler der SIMS-Messungen abzuschätzen betrachten wir das experimentell erhaltene Isotopenverhältnis aus einer Messung an einer Schicht mit einer hohen Gd-Konzentration von $2 \cdot 10^{20} \mathrm{~cm}^{-3}$. In Tabelle 3.1 ist dieses zusammen mit den Literaturwerten und der Abweichung der Messung von diesen aufgelistet. Der Fehler liegt bei 3 - $4 \%$. Als maßgeblich wird die ${ }^{160} \mathrm{Gd}$ Linie angenommen und der Fehler zu $3 \%$ bestimmt.

\subsection{Auswertung des Standards}

Abbildung 3.2(b) zeigt einen Vergleich des fertig ausgewerteten Standards aus den SIMSMessungen zusammen mit dem simulierten TRIM Profil aus Abbildung 3.1(b). Beim Vergleich der Messung und der Simulation fällt deutlich auf, daß das gemessene Profil in die Tiefe ausgeschmiert ist. Die Ausschmierung kann durch zwei Punkte erklärt werden. Zuerst berücksichtigt die TRIM Simulation nicht die Kristallstruktur des GaN, sondern nur die Massen der einzelnen Komponenten (Ga und N) und die Dichte des Materials. Das Wurzitgitter hat aber entlang der c-Achse offene Kanäle, die während der Implantation für erhöhte Eindringtiefen der Ionen sorgen können. Dieser Effekt wird durch eine Verkippung der c-Achse gegen die Implantationsrichtung minimiert. Der zweite Effekt ist ein systematischer Fehler der Meßmethode. Der Primärstrahl des SIMS trägt während des Sputterns Ionen mit in tiefere Schichten und schmiert so ein 
Profil in die Tiefe aus. Dieser Effekt läßt sich leider nicht vermeiden und ist als der Hauptgrund für das Ausschmieren anzuführen. Die ersten Messwerte (0-8nm) reißen nach oben aus (systematischer Fehler der SIMS). Diese Werte wurden für die Quantifizierung der Konzentrationen nicht beachtet.

Die Auswertung des Standards ist in Abbildung A.1 als Blockdiagramm gezeigt. Die Rohdaten werden zuerst auf eine starke Ga-Linie $\left({ }^{71} \mathrm{Ga}\right)$ normiert. Diese Normierung ist notwendig, da apparativ bedingt Sputterraten von Messung zu Messung nicht konstant gehalten werden können. Damit variieren die absoluten Sputterraten, aber nicht die Verhältnisse der verschiedenen Massen. Nach der Normierung werden Masseninterferenzen abgezogen. Masseninterferenzen sind in Implantationsprofilen leicht zu erkennen und zu quantifizieren, da wie in Abbildung 3.2(a) zu erkennen das Profil nicht auf den Untergrund abfällt. Zur Korrektur wurde für eine Tiefe von ca. 0,3-1,3 $\mu \mathrm{m}$ eine Gerade mit Steigung Null angefittet und dann abgezogen. Damit stellen die Linien für die Massen 155, 156 und 157 nun nur noch die entsprechenden Signale der Gd-Isotope dar. Die Messung verläuft immer in einzelnen (Sputter-) Cycles, die jeweils eine bestimmte Tiefe repräsentieren. Die Signale der einzelnen Gd-Isotope können nun für jeden Cycle einzeln zu einem Gesamt-Gd-Profil aufsummiert werden. Mit Hilfe einer Tiefenkalibrierung und der Gd-Fluenz der Implantation kann eine durchschnittliche Konzentration der Probe bestimmt werden. Für die Tiefenkalibrierung wird der Sputterkrater mit einem Dektak Oberflächenprofilometer vermessen und so den einzelnen Cycles eine Tiefe zugewiesen. Die durchschnittliche Konzentration berechnet sich aus Fluenz und einem willkürlich zu wählenden Tiefenabschnitt des Standards. Die Wahl des Tiefenabschnitts ist nur an die Bedingung gebunden, daß sich alle implantierten Gd-Atome in diesem Bereich befinden müssen. Es wurde hier der Bereich von 15-200 nm gewählt. Zusammen mit dem durchschnittlichen Signal aus demselben Tiefenbereich berechnet sich ein Faktor, welcher das Verhältnis des Gd-Signales und der ${ }^{71} \mathrm{Ga}$ Linie mit der Gd-Konzentration verknüpft. Mit Hilfe des Faktors und dem Gesamt-Gd-Profil kann jetzt das Konzentrationsprofil erstellt werden.

\subsection{Auswertung der Proben}

Aus der Auswertung des Standards steht ein Faktor zur Verfügung, der an das Verhältnis von Gd-Signal zur ${ }^{71} \mathrm{Ga}$-Linie multipliziert die Gd-Konzentration ergibt. Notwendigerweise müssen also auch bei der Auswertung der Proben die Gd-Signale auf die ${ }^{71} \mathrm{Ga}$ Linie normiert werden (Abbildung A.2). Ein Problem ist die Korrektur der Masseninterferenzen. Im Standard sind die Masseninterferenzen gut zu quantifizieren, da Bereiche mit und ohne Gd-Signal zur Verfügung stehen. In den Proben ist aber eine homogenere Verteilung des Gd durch die ganze Schicht zu erwarten und damit kein so offensichtlicher 
Maßstab für die Masseninterferenzen gegeben. Die Masseninterferenzen werden daher mit folgenden Annahmen quantifiziert.

Zuerst wird ein durchschnittliches Signal für jede einzelne Gd-Linie (noch inklusive der Masseninterferenzen) gebildet. Um die Masseninterferenzen zu quantifizieren wird das Verhältnis der Linien ${ }^{69} \mathrm{Ga}_{2},{ }^{69} \mathrm{Ga}^{71} \mathrm{Ga},{ }^{71} \mathrm{Ga}_{2}$ und die Annahme, daß die wesentliche Masseninterferenz die verschiedenen Linien der Ga-N-Cluster sind, benutzt (Tabelle 3.1). Die drei $\mathrm{Ga}_{2}$ Linien werden so normiert, daß die ${ }^{69} \mathrm{Ga}_{2}$ Linie der Linie mit Masse 152 entspricht. Der Isotopenanteil des ${ }^{152} \mathrm{Gd}$ von $0,2 \%$ wird als vernachlässigbar angenommen. So erzeugt man aus den ${ }^{69} \mathrm{Ga}^{71} \mathrm{Ga}$ und ${ }^{71} \mathrm{Ga}_{2}$ Linien die erwarteten Signale für die Massen 154 und 156. Diese können jetzt von den gemessenen Signalen der Massen 154 und 156 abgezogen werden und man erhält das reine Gd-Signal von ${ }^{154} \mathrm{Gd}$ und ${ }^{156} \mathrm{Gd}$. Danach wird für jede Masse das durchschnittliche Signal erstellt und zusammen mit dem Faktor aus der Auswertung des Standards die durchschnittliche Gd-Konzentration errechnet. Zusätzlich erhält man noch das Isotopenverhältnis der Gd-Isotope. Dies kann als Test für die Vertrauenswürdigkeit der Messung herangezogen werden.

\subsection{Ergebnisse in den beiden Serien}

Abbildung 3.3 zeigt ein repräsentatives Tiefenprofil des integrierten Gd-Signals. Die Gd-Konzentration ist homogen über die ca. $530 \mathrm{~nm}$ dicke Schicht verteilt. Eine leichte Erhöhung des Signals für Tiefen größer ca. $200 \mathrm{~nm}$ ist zu erkennen. Die homogene Verteilung des Gd aus den SIMS Daten steht in guter Übereinstimmung mit den XANESund EXAFS-Messungen (extended x-ray absorption fine structure) aus Abschnitt 5.2.

Für die beiden hergestellten Serien wurden unterschiedliche Wachstumsraten gefunden (Abschnitt 2.5). Geht man von einem vollständigen Einbau des während des Wachstums angebotenen Gd aus, so sollte bei konstanter Gd-Zellentemperatur eine lineare Abhängigkeit zwischen Gd-Konzentration und Wachstumsrate bestehen. Diese Annahme ist notwendig, da der Fluß der Gd-Zelle nicht bestimmt werden konnte. Aufgrund des hohen Dampfdruckes von Gd ist dieser so niedrig, daß der BEP nicht vom Hintergrunddruck unterscheidbar ist. Der hohe Dampfdruck macht aber auch die Annahme eines vollständigen Einbaus sinnvoll, da die Desorption bei Wachstumstemperatur vom Substrat vernachlässigbar ist. In Abbildung 3.4 ist die Wachstumsrate gegen die Gd-Konzentration aufgetragen. Die verschiedenen Farben der Punkte kodieren die Gd-Zellentemperatur. Die diskutierte Abhängigkeit kann durch diese Auftragung weder bestätigt noch widerlegt werden. Vielmehr kann festgestellt werden, daß die GdKonzentration stark streut und teilweise Unterschiede in der Konzentration von mehr als einer Größenordnung bei gleicher Gd-Zellentemperatur zu verzeichnen sind.

Abbildung 3.5 zeigt das Ergebnis der SIMS-Messungen in Abhängigkeit von der Gd- 


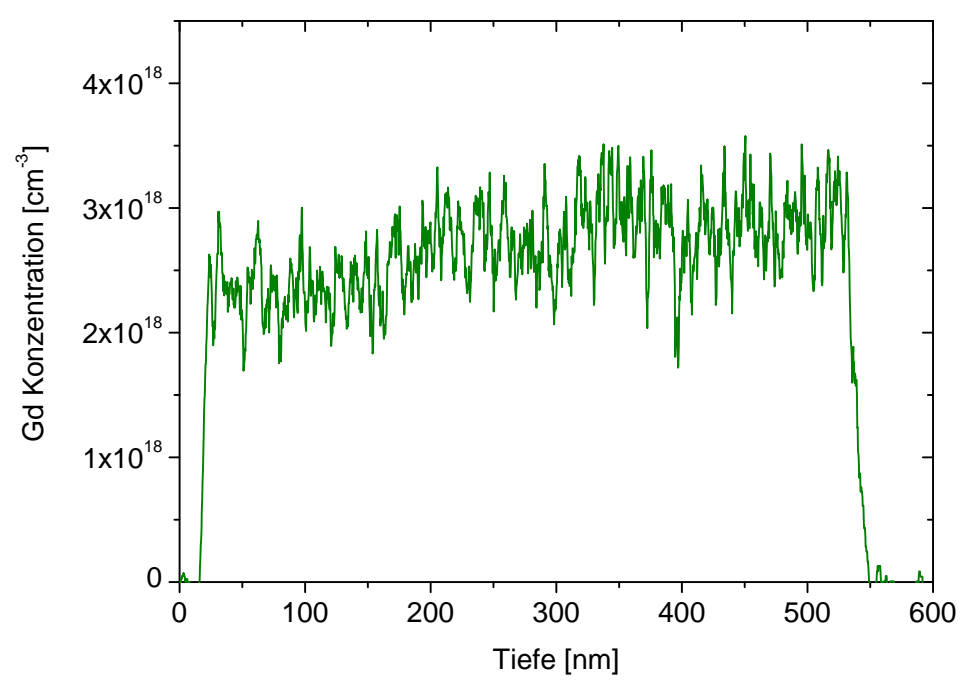

Abbildung 3.3: Gd-Tiefenprofil der Probe J0261, Probenoberfläche bei $x=0$.

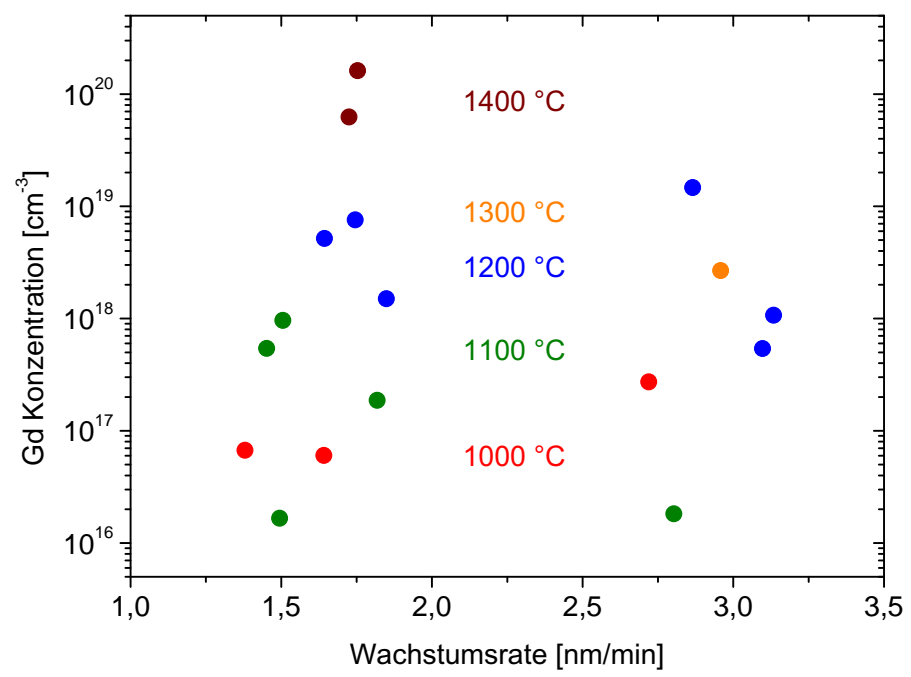

Abbildung 3.4:

Abhängigkeit der Gd Konzentration von der Wachstumsrate. Die Farbe der Punkte codiert die Gd Zellentemperatur. 


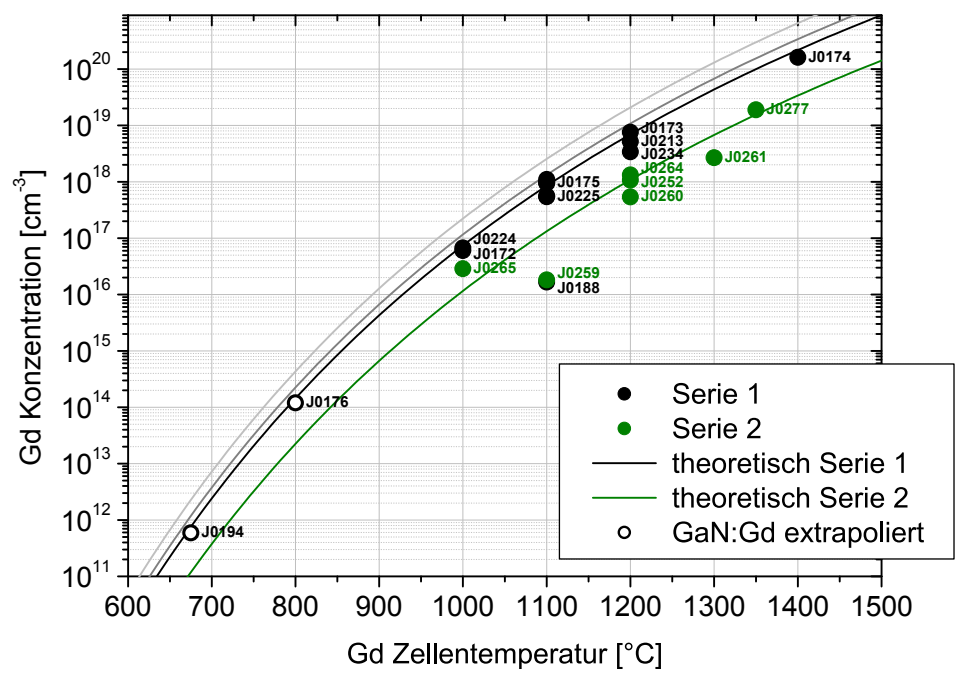

\begin{abstract}
Abbildung 3.5:
Gd-Konzentrationen der Proben aus den SIMS-Messungen gegen die Gd-Zellentemperatur aufgetragen. Die theoretischen Kurven sind nach Formel 3.1. Diskussion der verschiedenen Varianten siehe Text. Für Gd-Zellentemperaturen kleiner $1000^{\circ} \mathrm{C}$, bzw. Gd-Konzentrationen kleiner $10^{16} \mathrm{~cm}^{-3}$ sind die Konzentrationen durch die Kurven abgeschätzt. Geschlossene Symbole sind gemessene Werte, offene Symbole stehen für geschätzte Konzentrationen.
\end{abstract}

Zellentemperatur. Die gemessenen Werte (volle Symbole) überspannen einen Bereich von ca. $10^{16}$ bis $10^{20} \mathrm{~cm}^{-3}$. Die niedrigste gemessene Konzentration ist $2 \cdot 10^{16} \mathrm{~cm}^{-3}$. Die eingezeichneten Kurven sind theoretische Abschätzungen zur Gd-Konzentration und werden im folgenden Abschnitt diskutiert.

\title{
3.7 Theoretische Abschätzung der Gd Konzentration
}

Da die Aufösungsgrenze der Messmethode bei ca. $2 \cdot 10^{16} \mathrm{~cm}^{-3}$ liegt, können Proben mit einer Gd-Zellentemperatur von $1000^{\circ} \mathrm{C}$ und weniger nicht mit SIMS aufgelöst werden. Zur Abschätzung von geringen Gd-Konzentration wird die Theorie der Effusion herangezogen. Eine kurze Einführung zu diesem Thema findet sich in Anhang B.

Will man die Gd-Konzentration anhand der Gd Zellentemperatur abschätzen, so benötigt man den von der Zelle ausgehenden Teilchenfluß. Standardmäßig wird dieser bei der MBE durch den BEP repräsentiert. Da dieser aber in der verwendeten Temperaturspanne der Gd-Zelle nicht vom Hintergrunddruck zu unterscheiden ist, muß dieser abgeschätzt werden. Die Effusionstheorie liefert eine Formel für die Berechnung des Teilchenflusses auf der Probenoberfläche (Gleichung B.6). Teilt man diese durch die Wachstumsrate $\tau$, so ergibt sich bei der Annahme eines vollständigen Einbaus in die 
GaN-Matrix die Gd-Konzentration.

$$
c_{G d}=\frac{N_{G d}}{\tau}=\sqrt{\frac{N_{A}}{2 \pi^{3} k_{B} M T}} \cdot \frac{p_{0} A}{r^{2} \tau} \cdot \cos \phi \cdot e^{-\frac{\Delta H_{v a p}}{R}\left(\frac{1}{T}-\frac{1}{T_{0}}\right)}
$$

Für eine Herleitung der Formel ist auf Kapitel B verwiesen, dort sind auch die verwendeten Werte in Tabelle B.1 aufgeführt. Die aus Gleichung 3.1 resultierende Kurve ist in Abbildung 3.5 in hellgrau für Serie 1 (Wachstumsrate: $\tau_{1}=1,5 \mathrm{~nm} / \mathrm{min}$ ) und in dunkelgrau für Serie 2 (Wachstumsrate $\tau_{2}=2,9 \mathrm{~nm} / \mathrm{min}$ ) eingezeichnet. Wie in Kapitel B erläutert hängt der Fluß stark von der Geometrie des Zellentiegels und dessen Füllstand ab. Abbildung B.2(b) zeigt vergleichend eine Punktkalibrierung und die theoretische Kurve des BEP für die Ga-Zelle. Selbst bei der Verwendung der Korrekturen für nicht ideale Knudsenzellen (Kapitel B) ist eine Abweichung von ca. einer Größenordnung zu erkennen. Um aus den theoretischen Kurven eine Abschätzung für die Gd-Konzentration bei niedrigeren Zellentemperaturen zu bekommen wurden diese mit einen empirischen Faktor $\mathrm{C}_{\mathrm{emp}}$ multipliziert, um die Ergebnisse der SIMS-Messungen widerzuspiegeln. In Abbildung 3.5 sind diese verschobenen Kurven als theoretisch Serie 1 und theoretisch Serie 2 eingezeichnet. Dabei wurde für Serie 1 der Faktor zu $\mathrm{C}_{\mathrm{emp}} \approx \frac{1}{3}$ (schwarze Kurve) und für Serie $2 \mathrm{zu} \mathrm{C} \mathrm{e}_{\mathrm{emp}} \approx \frac{1}{10}$ (grüne Kurve) bestimmt. Proben für welche die GdKonzentration auf diese Art und Weise abgeschätzt wurde sind in Abbildung 3.5 als offene Symbole dargestellt.

\subsection{Verunreinigungen}

Oft stammen Verunreinigungen in MBE- oder MOCVD-hergestellten Halbleiterschichten aus dem Quellenmaterial. Das in dieser Arbeit verwendete Gd hat eine Reinheit von $4 \mathrm{~N}$. Verglichen mit den anderen Quellenmaterialien, welche Reinheiten zwischen 6N - 7N haben, enthält das Gd viele Fremdatome.

Um den Einfluß von Übergangsmetallen aus dem Quellenmaterial auszuschließen wurde eine Schicht mit hoher Gd-Konzentration (J0174, $\mathrm{c}_{\mathrm{Gd}}=1,5 \cdot 10^{20} \mathrm{~cm}^{-3}$ ) mit TOFSIMS auf die Reihe der Übergansgsmetalle untersucht. Abbildung 3.6 zeigt das Massenspektrum im Bereich der Übergangsmetalle und der Gd-Isotope. Die Daten sind nicht bezüglich Masseninterferenzen korrigiert, allerdings ist die Gd-Konzentration mit ca. 0,23\% so hoch, daß die Signale von 154 bis 158 u nur einen vernachlässigbaren Anteil an Interferenzen enthalten. Vergleicht man die Verhältnisse der Signale zwischen 154 bis $160 \mathrm{u}$ mit dem Isotopenverhältnis von Gd, so ist dieses in guter Übereinstimmung mit den Literaturwerten (Tabelle 3.1).

Die Aufösungsgrenze für Gd wurde schon in Abschnitt $3.3 \mathrm{zu} 2 \cdot 10^{16} \mathrm{~cm}^{-3}$ bestimmt. Für eine Abschätzung der Auflösungsgrenze der Übergangsmetalle wurden Messungen 


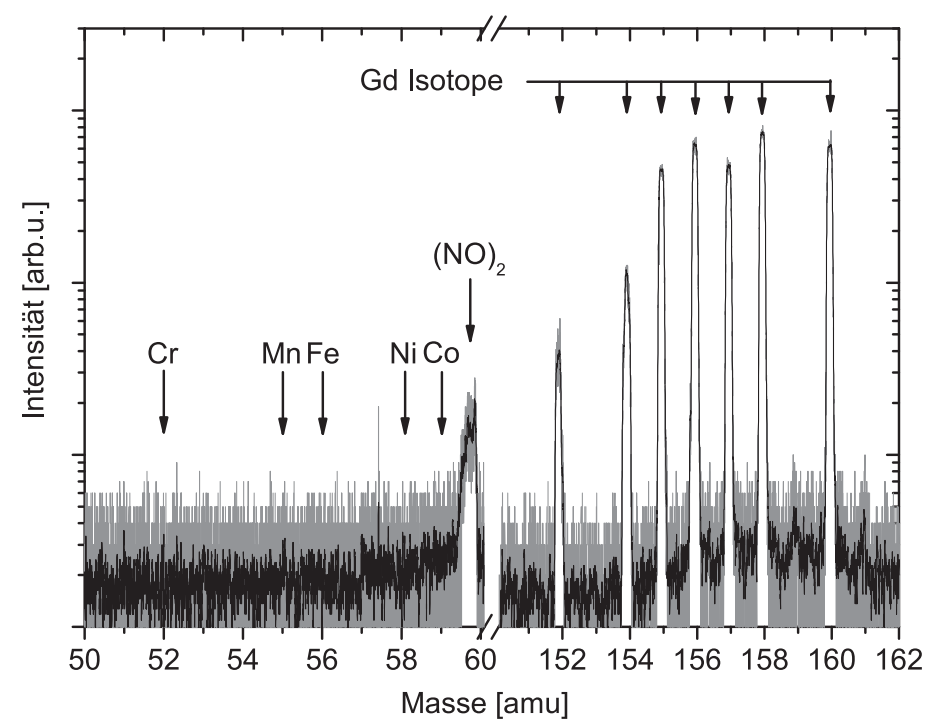

\section{Abbildung 3.6:}

TOF SIMS Spektrum einer GaN:Gd Schicht mit $1,5 \cdot 10^{20} \mathrm{~cm}^{-3}$ Gd Konzentration. Die graue Kurve zeigt die Rohdaten. Zwischen 50 und 60 amu sind keine Signale von Übergangsmetallen zu erkennen. Zwischen 152 und 160 amu ist deutlich die Signatur der Gd Isotope erkennbar. Die schwarze Kurve ist eine 10-Punkt Glättung der Rohdaten (10 point adjacent averaging smoothing). Auch dort sind keine im Untergrund versteckten Signale zu erkennen.

an GaN:Mn-Schichten herangezogen. Hier können Konzentrationen von $10^{17} \mathrm{~cm}^{-3}$ sicher aufgelöst werden. Betrachtet man wieder Abbildung 3.6, so sind dort keine Signale im Bereich der Übergangsmetalle zu erkennen. Auch nach einer Glättung der Kurve durch eine 10-Punkt Glättung (schwarze Kurve) sind keine Signale zu erkennen. Damit ist die Konzentration der Übergangsmetalle in dieser Schicht deutlich unterhalb der $10^{17} \mathrm{~cm}^{-3}$ und mindestens drei Größenordnungen kleiner als die Gd-Konzentration. Nimmt man weiter an, daß die größten Verunreinigungen im Bereich der Übergangsmetalle aus dem Gd-Quellenmaterial stammen (4N Reinheit), so ist dieser Faktor 1000 auf alle Gd-Dotierten GaN-Schichten anwendbar, da dann auch die Verunreinigungen mit der Gd-Quellentemperatur skalieren. Das Aufschwimmen von Verunreinigungen im Quellenmaterial ist auszuschließen, da mit zwei Ausnahmen alle Schichten unterhalb der Schmelztemperatur von Gd hergestellt wurden (ca. $1312^{\circ} \mathrm{C}$ ). 


\section{Zusammenfassung}

Die Auswertung der TOF-SIMS-Spektren hat sich als komplexes Thema herausgestellt. Für alle Gd-Isotope außer dem ${ }^{160} \mathrm{Gd}$ sind Masseninterferenzen zu finden. Diese können je nach Stärke des Signales gut quantifiziert und korrigiert werden. Die Aufösungsgrenze des TOF-SIMS für Gd wurde zu $10^{16} \mathrm{~cm}^{-3}$ und der Fehler zu $3 \%$ bestimmt. Die homogene Verteilung des Gd in der GaN-Schicht konnte anhand von Tiefenprofilen bestätigt werden.

Die Betrachtung der Gd-Konzentration in Abhängigkeit von der Gd-Zellentemperatur zeigt Probleme bei der Reproduktion von Gd-Konzentration durch die alleinige Steuerung anhand der Gd-Zellentemperatur. Gd-Konzentrationen in Schichten mit gleichen Wachstumsparametern zeigen Unterschiede in der Gd-Konzentration von bis zu einer Größenordnung. Die Vermutung eines bis jetzt unkontrollierten Parameters liegt hier nahe. Hierbei könnte es sich um Änderungen im Füllgrad der Gd-Zelle (die Zelle wurde zwischenzeitlich neu befüllt) oder subtilere Änderungen handeln. Spekulationen über den Sauerstoffgehalt im Restgas werden durch Abschnitt 4.4.3 gerechtfertigt. Eine exakte Kontrolle der Wachstumsparameter ist daher von bedeutender Rolle für die Herstellung von GaN:Gd.

Die SIMS-Messungen zeigen, daß GaN:Gd mit Gd-Konzentrationen im Bereich von $10^{16}$ bis $10^{20} \mathrm{~cm}^{-3}$ hergestellt wurde. Mit Hilfe der Effusionstheorie gibt die theoretische Abschätzung der Gd-Konzentration eine gute Näherung der Konzentrationen unterhalb von $10^{16} \mathrm{~cm}^{-3}$. Hier werden sehr niedrige Gd-Konzentrationen von $10^{12} \mathrm{~cm}^{-3}$ abgeschätzt.

Eine Abschätzung der Verunreinigungskonzentrationen von Übergangsmetallen aus dem Gd-Quellenmaterial anhand einer hoch Gd-dotierten Schicht ergibt, daß alle Übergangsmetalle mit einer mindestens um den Faktor 1000 kleineren Konzentration vorliegen als das Gd. 


\section{Kapitel 4}

\section{Magnetische Eigenschaften}

Dieses Kapitel befaßt sich mit den magnetischen Messungen und Eigenschaften von GaN:Gd und dem Einfluß von co-Dotierungen mit Silizium, Wasserstoff und Sauerstoff auf diese. Nach einer ausführlichen Diskussion der SQUID-Messungen und möglichen Fehlern dabei (Abschnitt 4.1) werden die Messungen an den verschiedenen Proben präsentiert (Abschnitt 4.2 - 4.4). Anschließend wird in Abschnitt 4.5 und 4.6 das Bild zusammengefaßt und diskutiert. Abschließend werden in den Abschnitten 4.7 und 4.8 die Charakteristika von Ausscheidungen und die magnetische Probenalterung untersucht. 


\subsection{SQUID Messungen}

Die magnetischen Messungen wurden mit einem Quantum Design MPMS 5 XL SQUID Magnetometer durchgeführt. Als Aufösungsgrenze gibt Quantum Design $10^{-7} \mathrm{emu}$ an. Durch das hohe Aufösungsvermögen ist das SQUID ein gutes Werkzeug um die hier vorliegenden kleinen magnetischen Momente zu messen. Es muß aber immer vor Augen gehalten werden, daß die hier untersuchten Schichten Magnetisierungen nahe der Auflösungsgrenze des SQUID haben und somit schon kleinste Verunreinigungen oder systematische Fehler bei der Messung einen großen Einfluß auf die Ergebnisse haben können.

Die vom SQUID verwendete Einheit für die Magnetisierung ist das emu. Eine deutlich vertrautere Größe für die Betrachtung von Magnetisierungen ist $\mu_{\mathrm{B}}$ statt $\mathrm{emu}^{1}$. Mit der Kenntnis der Gd-Konzentration lassen sich so sehr schnell Vergleiche von Magnetisierungen und Verunreinigungs- oder Defektkonzentrationen anstellen. Die absolute Magnetisierung der Probe in emu bzw. $\mu_{\mathrm{B}}$ ist eine vom Volumen abhängige Größe. Um Vergleiche der Schichten untereinander zu ermöglichen wird diese Größe auf das Volumen in $\mathrm{cm}^{3}$ bezogen. Die verwendete Einheit ist daher $\mu_{\mathrm{B}} / \mathrm{cm}^{3}$. Die effektiven Magentisierungen sind auf die Gd-Konzentration bezogen und werden in $\mu_{\mathrm{B}}$ pro Gd-Atom angegeben.

\subsubsection{Systematische Fehler bei SQUID-Messungen}

In Abschnitt 3.8 wurde bereits die mögliche Verunreinigung der Schicht mit Übergangsmetallen aus dem Quellenmaterial untersucht. Magnetische Verunreinigungen können aber auch bei falscher Handhabung der Proben entstehen, beispielsweise durch Benutzung von metallischen Pinzetten. Es kann so zu Eisen-, Chrom- oder Nickelverunreinigungen kommen, welche im Vergleich zu den hier zu messenden Signalen starke parasitäre magnetische Momente erzeugen können. Es ist bei der Handhabung der Proben darauf zu achten nur nicht-magnetische Werkzeuge, wie z.B. Teflonpinzetten, zu benutzen. Zur Minimierung solcher Verunerinigungen wurden die Proben vor jeder Messung gründlich mit Aceton und Propanol gereinigt und mit Salzsäure geätzt.

Besonders bei der Messung von kleinen Signalen ist auch das Wissen über den verwendeten Probenhalter von großer Bedeutung. Bauartbeding mißt das SQUID Unterschiede in der Magnetisierung und ist für homogen verteilte Momente nicht sensitiv. So kann unter anderm gewährleistet werden, daß das äußere angelegte Feld nicht mitgemessen wird. Diese Eigenschaft hat aber auch zur Folge, daß ein inhomogenes diamagnetisches Signal zu einem parasitären Signal führen kann. Die von Quantum Design als Probenhalter gelieferten Strohhalme weisen zum Teil solche Inhomogenitäten auf. Diese entstehen

\footnotetext{
${ }^{1} 1 \mu_{\mathrm{B}}=9,274 \cdot 10^{-24} \mathrm{Am}^{2}=9,274 \cdot 10^{-21} \mathrm{emu}$ (CODATA recommended values of the fundamental physical constants: 2006)
} 


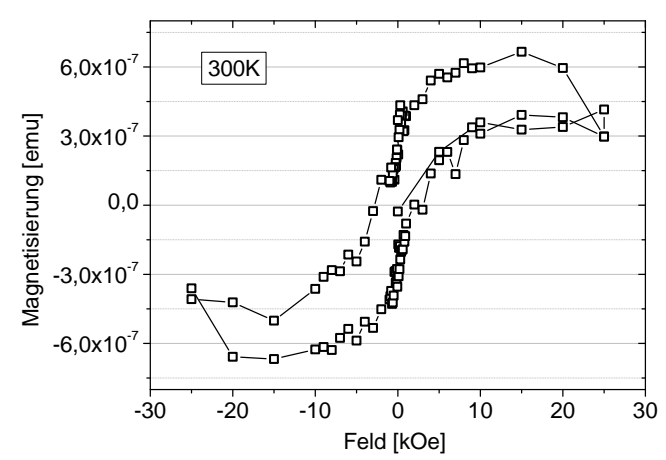

Abbildung 4.1:

Beispiel einer trapped field "Hysterese". Charakteristisch für dieses Artefakt ist die sich immer beim höchsten Meßfeld öffnende Kurve.

z.B. wenn Bindungen innerhalb des Polymers aufgebrochen werden. Scharfe Knicke oder ähnliche Beschädigungen des Halmes führen deshalb zu einem systematischen Fehler bei der Messung, welche sogar den Anschein eines Ferromagneten erwecken können [57]. Um solche Fehler zu vermeiden wurden die Strohalme immer äußerst pfleglich behandelt.

Auch die verwendeten Substrate können Verunreinigungen beherbergen, welche magnetische Momente tragen. Jedes verwendete Substrat wurde ohne und/oder mit einer undotierten GaN-Schicht im SQUID gemessen und somit der Einfluß von Signalen aus dem Substrat ausgeschlossen.

Ein weiteres Problem bei der Messung kleiner Signale im SQUID ist das sog. trapped field. Dieser systematische Fehler des angelegten äußeren Feldes ist auf das Einfangen von magnetischem Fluß innerhalb der supraleitenden Spulen zurückzuführen. Es handelt sich dabei um ein charakteristisches Verhalten eines Typ 2 Supraleiters und führt zu einer feld- und Vorgeschichten-abhängigen Abweichung des tatsächlich angelegten Feldes vom Sollwert. Abbildung 4.1 zeigt einen typischen Kurvenverlauf einer solchen „Hysterese“. Die Größe der Hysterese ist dabei von der Meßprozedur und dem diamagnetischen Signal der Probe, also implizit vom Volumen der Probe abhängig. Charakteristisch für eine solche trapped field Hysterese ist die Öffnung der Kurve an den Umkehrpunkten bei maximalem äußeren Feld. Die Aufspaltung findet immer beim höchsten gemessenen Feld statt. Bei nicht eindeutigen Messungen kann so eine Änderung der Meßprozedur Aufschluß darüber geben, ob es sich um ein Artefakt handelt oder ein echtes Signal aus der Probe.

Die durch Rauschen in der Messung entstehenden statistischen Fehler im SQUID sind aufgrund von vielen Wiederholungen der einzelnen Meßpunkte sehr klein. Aus diesem Grund sind in den folgenden Auftragungen keine Fehler gezeigt. Die Grenze, ab welcher ein gemessenes Signal als Artefakt betrachtet wird, wurde anhand eines Vergleiches 
von der in Abbildung 4.1 gezeigten Messung und den jeweiligen Meßdaten bestimmt und beläuft sich auf ca. $3 \cdot 10^{-7} \mathrm{emu}$. Die Auftragungen im folgenden sind in $\mu_{\mathrm{B}} / \mathrm{cm}^{3}$. Auch wenn die Zuordnung einer auf das Volumen bezogenen Größe nicht ganz eindeutig ist, da die Volumina der verschiedenen Proben variieren, kann ca. $5 \cdot 10^{18} \mu_{\mathrm{B}} / \mathrm{cm}^{3}$ als Artefaktgrenze angegeben werden. Diese Grenze ist in den entsprechenden Auftragungen eingezeichnet.

\subsubsection{Meßprozedur}

Die Meßprozedur für die magnetischen Messungen startet mit der im Nullfeld abgekühlten temperaturabhängigen Messung (zero field cooled, zfc). Die Probe wird ohne äußeres Feld auf $4 \mathrm{~K}$ abgekühlt und dann bei einem äußeren Feld von 100 Oe die Magnetisierung während der Erwärmung auf $300 \mathrm{~K}$ gemessen. Es ist sinnvoll diese Messung an den Anfang der Sequenz zu stellen, da die Vorgeschichte der Probe, besonders angelegte Sättigungsfelder eine Auswirkung auf diese Messung haben können. Ist die Vorgeschichte der Probe unklar oder wurde sie schonmal hohen Feldern ausgesetzt, so wird eine Entmagnetisierung durchgeführt. Dazu wird die Probe immer kleiner werdenen Feldern mit alternierendem Vorzeichen startend bei +30 kOe ausgesetzt. Die Abstände der Felder werden dabei zu kleinen Feldern immer kleiner. Als zweite Messung folgt die field cooled Messung (fc). Die Probe wird jetzt im Sättigungsfeld von $25 \mathrm{kOe}$ auf $4 \mathrm{~K}$ abgekühlt und die Magnetisierung bei 100 Oe äußerem Feld während der Erwärmung auf $300 \mathrm{~K}$ gemessen. Nach den temperaturabhängigen Messungen folgen die feldabhängigen Messungen.

\subsubsection{Korrekturen der Rohdaten}

Der diamagnetische Hintergrund wurde bei allen Messungen korrigiert. Dazu werden bei der feldabhängigen Messung bei $300 \mathrm{~K}$ die absoluten Meßwerte zwischen 6 und $25 \mathrm{kOe}$ (pos. und neg. Feld) betrachtet. Ein linearer Fit dieser Werte liefert die Steigung der diamagnetischen Gerade. Werte bei kleineren Feldern werden aufgrund von möglichen, dort nicht gesättigten ferromagnetischen Beiträgen ausgelassen. Für die Korrektur des diamagnetischen Untergrundes bei feldabhängigen Messungen unterhalb von Raumtemperatur wird auch die Steigung des Fits bei $300 \mathrm{~K}$ verwendet. Dadurch wird ausgeschlossen, daß eine durch einen paramagnetischen Anteil entstandene Steigung fälschlicherweise mit abgezogen wird. Diese Vorgehensweise ist sinnvoll, da im Laufe der Arbeit gezeigt werden konnte, daß eventuell bei $300 \mathrm{~K}$ vorhandene superparamagnetische Anteile an der Magnetisierung auszuschließen sind (Abschnitt 4.7). Auch für die Korrektur der temperaturabhängigen Messungen wird diese Steigung verwendet. Kleinste Veränderungen am Probenhalter (anderer Strohhalm), der Probe (abgesplitterte Ecken) oder Verunreinigungen auf der Probe verändern das diamagnetische Signal. Dieses muß bei der 


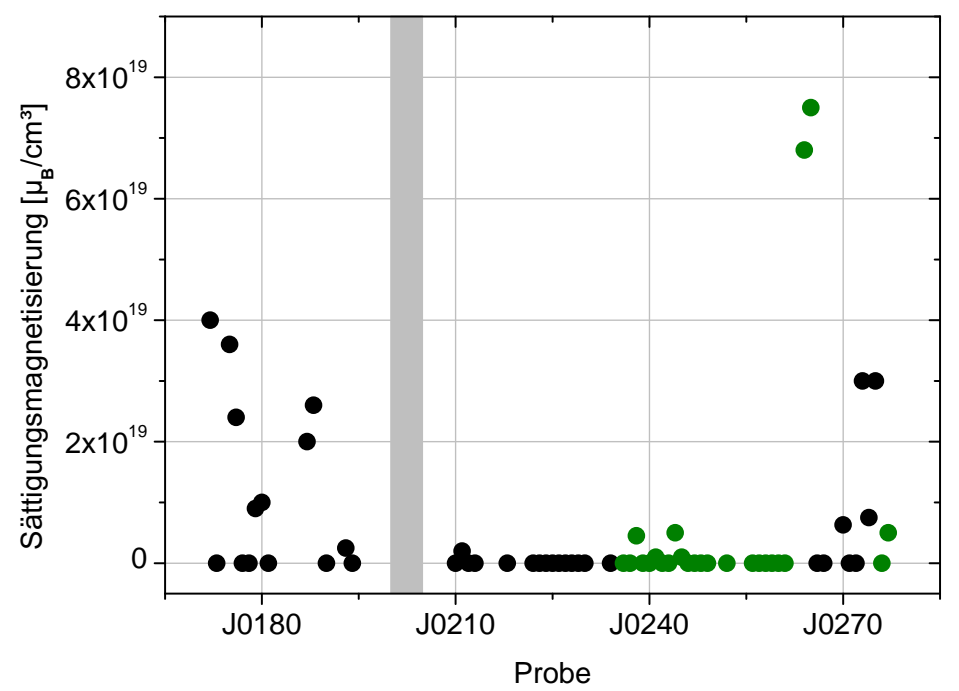

\begin{abstract}
Abbildung 4.2:
Sättigungsmagnetisierung gegen die Probennummer. Schwarze Symbole stellen Proben der Serie 1 dar, grüne Symbole Proben der Serie 2. Der graue Balken zeigt den Zeitraum der Wartung der Anlage.
\end{abstract}

Auswertung von Messungen einer Probe beachtet werden, welche zwischen den Messungen aus- und wieder eingebaut wurde.

Zur Korrektur des trapped field wird von den magnetischen Momenten jeder feldabhängigen Messung der zugehörige Wert aus der in Abbildung 4.1 gezeigten trapped field Hysterese abgezogen. Da die Stärke dieser auf die Stärke des diamagnetischen Signales zurückzuführen ist, werden die Korrekturdaten mit der diamagnetischen Steigung skaliert.

\title{
4.2 Übersicht und Reproduzierbarkeit
}

Im Laufe dieser Arbeit wurden ca. 80 Schichten (inkl. Referenzen und Kalibrierungen) hergestellt und unter anderem magnetisch charakterisiert. Ein Großteil der Gd-dotierten GaN-Schichten weist keine magnetische Ordnung auf. Selbst Reproduktionen von ferromagnetischen Proben liefern zum Teil Schichten ohne hysteretisches Verhalten. In Tabelle A.1 sind Reproduktionen und deren magnetisches Verhalten zusammengefaßt. Für eine erste Übersicht über die magnetischen Eigenschaften ist in Abbildung 4.2 die mit SQUID bestimmte Sättigungsmagnetisierung bei $300 \mathrm{~K}$ gegen die Probennummer aufgetragen. Die Serie 1 (schwarze Punkte) ist in drei unterschiedliche Phasen zu teilen. Innerhalb der ersten Phase (Proben links des grauen Bereiches) gibt es verschiedene 
Schichten mit ferromagnetischem Verhalten. Nach der Generalüberholung der Stickstoffplasmaquelle und Wartungsarbeiten an der Anlage (rechts des grauen Bereiches) konnten innerhalb der Serie 1 keine Schichten mit magnetischer Ordnung mehr hergestellt werden. Die dritte Phase der Serie 1 sind Schichten mit höherer Probennummer ( $\approx J 0270)$. Auch für diese ist mit Ausnahme der Sauerstoff-co-dotierten GaN:Gd-Schichten kein ferromagnetisches Verhalten zu finden. In dieser dritten Phase wurde der exakte Rückbau der Änderungen an der Stickstoffplasmaquelle durchgeführt und versucht verschiedene Reproduktionen von ferromagnetischen Schichten herzustellen. Ein Einfluß der Plasmaquelle auf die magnetischen Eigenschaften kann so weitestgehend ausgeschlossen werden. Innerhalb der Serie 2 (grüne Punkte) folgen die magnetischen Eigenschaften keinem erkennbaren Trend. Auch die Variation der in Tabelle A.2 festgehaltenen Parameter wie Substrattemperatur und III-V-Verhältnis zeigen keinen korrelierbaren Einfluß auf die magnetischen Eigenschaften.

Die magnetischen Eigenschaften können allgemein als schlecht reproduziebar bezeichnet werden und Variationen der Wachstumsparameter und Änderungen an der Plasmaquelle zeigen keine Korrelation mit diesen. Ein solches Verhalten deutet darauf hin, daß ein bis jetzt nicht berücksichtigter Parameter eine wichtige Rolle für die magnetische Ordnung in GaN:Gd spielt. Auch ist zu erkennen, daß dieser Parameter sich nicht willkürlich von Wachstum zu Wachstum ändert, sondern eher einen globalen Charakter während des Wachstums hat und sich von Zeit zu Zeit ändert. Verschiedene Möglichkeiten sind wechselndes Quellenmaterial (insbesondere Gd), Hintergrunddruck von Verunreinigungen in der Kammer (z.B. Sauerstoff und Wasserstoff). Der Einfluß des Gd Quellenmateriales konnte schon in Abschnitt 3.8 ausgeschlossen werden. Auch konnte innerhalb der Serie 2 kein Einfluß des III-V-Verhältnisses oder der Substrattemperatur bei gezielter Variation nachgewiesen werden. Damit können auch Schwankungen der Materialflüsse ausgeschlossen werden. Die Einflüsse der co-Dotierungen werden in den folgenden Abschnitten ausführlicher diskutiert.

\subsection{Nicht co-dotiertes GaN:Gd}

Repräsentativ für Proben mit ferromagnetischem Verhalten ist die in Abbildung 4.3 gezeigte Feld- und Temperaturabhängigkeit der Schicht J0176. In der feldabhängigen Messung der Magnetisierung bei $300 \mathrm{~K}$ (Abbildung 4.3(a)) ist eine deutliche Sättigung für externe Felder größer $10 \mathrm{kOe}$ zu erkennen. Die Sättigungsmagnetisierung beträgt gut $2 \cdot 10^{19} \mu_{\mathrm{B}} / \mathrm{cm}^{3}$ und in der Vergrößerung ist eine deutlich geöffnete Hysterese mit einem Koerzitivfeld von ca. 100 Oe zu erkennen. Abbildung 4.3(b) zeigt die zugehörigen fc und zfc Messungen. Die fc Kurve liegt deutlich oberhalb der zfc Kurve und die Aufspaltung bei $300 \mathrm{~K}$ ist konsistent mit dem Remanenzfeld der Hysterese bei $300 \mathrm{~K}$. fc 


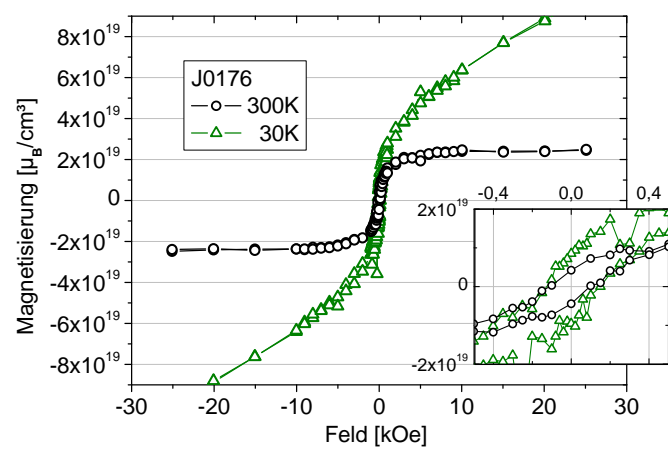

(a) Magnetisierung bei $300 \mathrm{~K}$

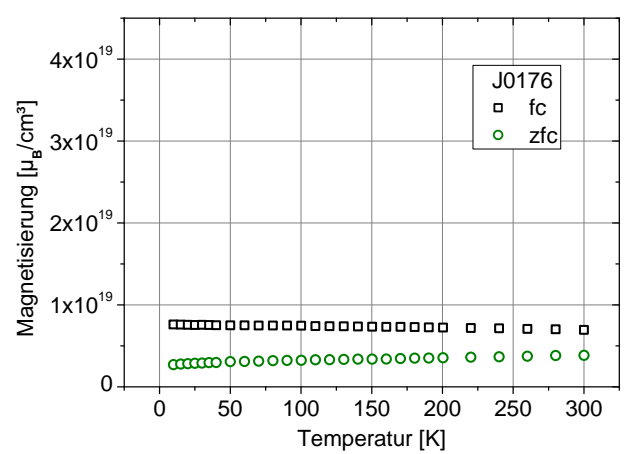

(b) Temperaturabhängigkeit der Magnetisierung

\section{Abbildung 4.3:}

Magnetisierung der Probe J0176. $\mathrm{T}_{\mathrm{Gd}}=800^{\circ} \mathrm{C}, \mathrm{c}_{\mathrm{Gd}}=10^{14} \mathrm{~cm}^{-3}$. (a) Feldabhängigkeit bei $300 \mathrm{~K}$, (b) Temperaturabhängigkeit bei einem Meßfeld von $100 \mathrm{Oe.}$

und zfc Kurve zeigen keine magnetischen Übergänge. Dieses deutet zusammen mit dem hysteretischen Verhalten bei $300 \mathrm{~K}$ auf Ferromagnetismus mit einer Curie Temperatur oberhalb von $300 \mathrm{~K}$ hin. Die Möglichkeit eines geblockten Superparamagneten durch Gd-haltige Ausscheidungen kann ausgeschlossen werden (Kapitel 5).

Für tiefe Temperaturen wird ein deutlicher paramagnetischer Anteil an der Gesamtmagnetisierung gefunden, welcher der ferromagnetischen Phase überlagert ist. Eine repräsentative tieftemperatur Hysterese ist in Abbildung 4.3(a) gezeigt. Auch dieses Verhalten wird in der Literatur von Dhar et al. und Hite et al. gefunden. Der von Dhar et al. in allen Schichten gefundene magnetische Übergang bei ca. $70 \mathrm{~K}$ kann nicht bestätigt werden (Abbildung 4.3(b) und Abschnitt 4.7). Das bei tiefen Temperaturen überlagerte paramagnetische Signal deutet darauf hin, daß nicht die gesamten Gd Atome an einer ferromagnetischen Phase beteiligt sind, sondern als isolierte Momente innerhalb der GaN Matrix vorliegen. Eine detailierte Analyse dieser isolierten Momente ist mit ausführlichen Messungen des Tieftemperaturverhalten der Proben möglich, war aber nicht Ziel der hier gezeigten Untersuchungen.

In Abbildung 4.4(a) ist ein Vergleich der Hysteresen von Schichten mit steigender GdKonzentration aus der Serie 1 gezeigt (J0194, J0176, J0172, J0175, J0173). Es ist deutlich der Trend einer steigenden Sättigungsmagnetisierung mit steigender Gd-Konzentration zu erkennen. Für die Schicht mit der höchsten Gd-Konzentration (J0173) wird kein Ferromagnetismus beobachtet. Schichten der Serie 2 zeigen dieses Verhalten deutlich ausgeprägter. In Abbildung 4.4(b) wird eine Folge von Schichten mit steigender GdKonzentration aus Serie 2 gezeigt (J0276, J0265, J0264, J0277). Die Sättigungsmagnetisierungen sind hier deutlich höher als in Proben der Serie 1. Auch hier ist für die Probe 


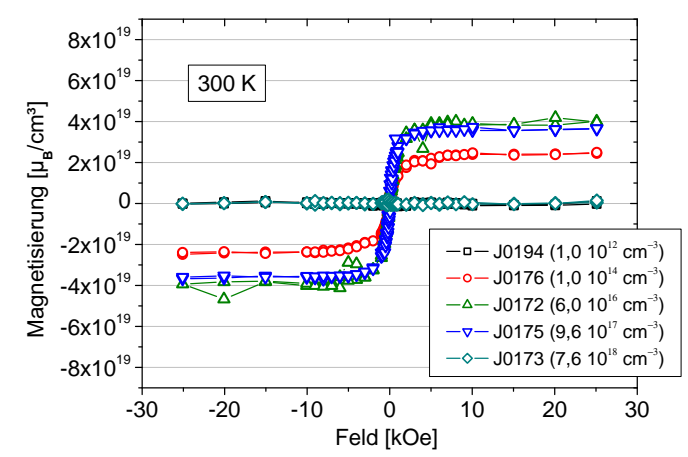

(a) Serie 1

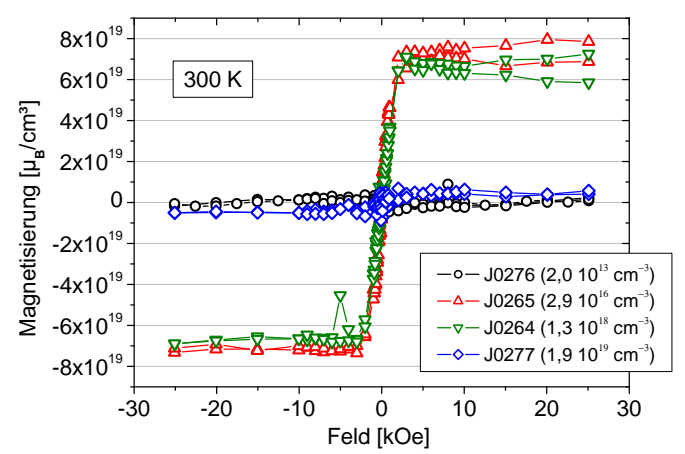

(b) Serie 2

\section{Abbildung 4.4:}

Vergleich der Hysteresen von nicht co-dotiertem GaN:Gd bei 300 K. (a) zeigt Proben der Serie 1 und (b) der Serie 2.

mit höchster Gd-Konzentration (J0277) keine Sättigungsmagnetisierung zu finden.

\subsection{Co-Dotierungen}

Dotierungen haben in Halbleitern großen Einfluß auf die elektrischen Eigenschaften. Auch die Defektlandschaft wird von einigen Dotierungen in GaN beeinflußt. So kann z.B. durch H-Dotierung die $\mathrm{V}_{\mathrm{Ga}}$-Bildung reduziert werden [39]. Verschiedene theoretische und experimentelle Arbeiten zeigen auch einen Einfluß dieser Eigenschaften auf die magentischen Eigenschaften von GaN:Gd (Kapitel 1). Um diese Einflüsse zu untersuchen wurden co-Dotierungen mit $\mathrm{Si}, \mathrm{H}$ oder O durchgeführt.

\subsubsection{Silizium-co-Dotierung}

Abbildung 4.5(a) zeigt die Magnetisierungen einer Reihe von Si-co-dotierten GaN:GdSchichten. Obwohl Probe J0190 mit einer Gd-Zellentemperatur von $1100{ }^{\circ} \mathrm{C}$ hergestellt wurde, ist kein Gd nachweisbar. Vergleicht man mit nicht co-dotierten Proben, so erwartet man eine Konzentration von ca. $7,5 \cdot 10^{17} \mathrm{~cm}^{-3}$. Die Gd-Konzentration ist deshalb mit $<2 \cdot 10^{16} \mathrm{~cm}^{-3}$ angegeben. Ein Einfluß der Si-Dotierung auf den Gd-Einbau in die GaN-Matrix kann nicht ausgeschlossen werden. Allgemein ist auch hier ein vergleichbares Verhalten wie bei den nicht co-dotierten Schichten zu beobachten. Niedrige und hohe Gd-Konzentrationen zeigen eine sehr schwache bis verschwindene Hysterese und nur für mittlere Gd-Konzentrationen wird eine Sättigungsmagnetisierung beobachtet. Allerdings verschwindet die Hysterese schon bei deutlich geringeren Gd-Konzentrationen als bei den nicht co-dotierten Schichten. Abschließend können hier noch keine eindeutigen 


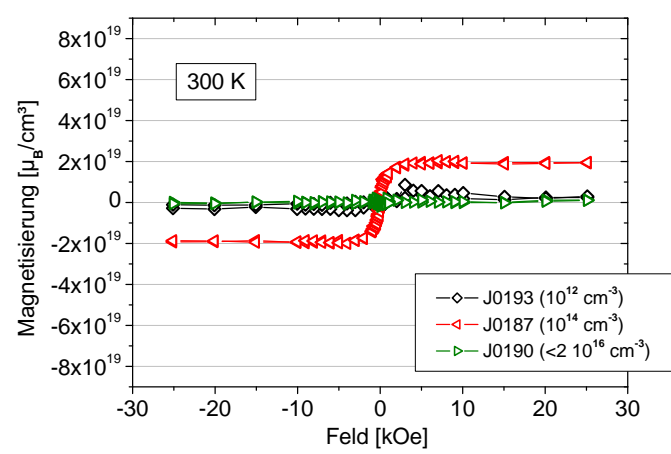

(a) Si-co-Dotierung

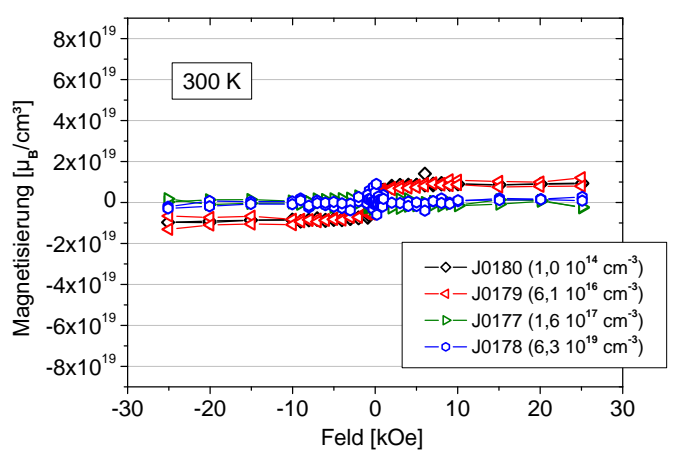

(b) H-co-Dotierung

\section{Abbildung 4.5:}

Vergleich der Hysteresen von co-dotiertem GaN:Gd mit Si oder H bei $300 \mathrm{~K}$. (a) zeigt Si-codotierte Proben und (b) zeigt H-co-dotierte Proben.

Schlüsse gezogen werden, da bis jetzt nur drei Proben zur Verfügung stehen.

\subsubsection{Wasserstoff-co-Dotierung}

Wasserstoff co-dotierte GaN:Gd-Schichten, wie in Abbildung 4.5(b) gezeigt, weisen eine deutlich reduzierte Magnetisierung im gesamten Gd-Konzentrationsbereich auf. Auch hier verschwindet für hohe Gd-Konzentrationen die Magnetisierung. Es scheint keine Abhängigkeit der Sättigungsmagnetisierung von der Gd-Konzentration zu bestehen. Die Trends der Si- und H-co-Dotierung von GaN:Gd werden in Abschnitt 4.5 zusammengefaßst und verglichen.

\subsubsection{Sauerstoff-co-Dotierung}

In Abbildung 4.6 sind die Hysteresen von drei Sauerstoff-co-dotierten GaN:Gd-Schichten gezeigt (J0273, J0274, J0275). Die Schichten unterscheiden sich im Sauerstoffangebot und wurden ansonsten mit identischen Wachstumsparametern hergestellt. Zum Vergleich sind die Hysteresen zweier weiterer Schichten in grau gezeigt. Die hellgraue Kurve ist die in Abbildung 4.4(a) gezeigte Probe J0175. Sie hat eine vergleichbare GdKonzentration, ist aber ca. 4 Jahre vor den Sauerstoff-co-dotierten Proben hergestellt worden. Die dunkelgraue Kurve gehört zu Probe J0267. Diese wurde zeitnah zu den Sauerstoff-co-dotierten Proben hergestellt und hat eine Gd-Konzentration von geschätzten $6 \cdot 10^{16} \mathrm{~cm}^{-3}$. Sie liegt damit im einem Bereich der Gd-Konzetration, welche bei Proben mit ferromagnetischem Verhalten die höchsten Magnetisierungen gezeigt hat.

Für die beiden Schichten mit hohem Sauerstoffangebot (J0273, J0275) ist eine nahe- 


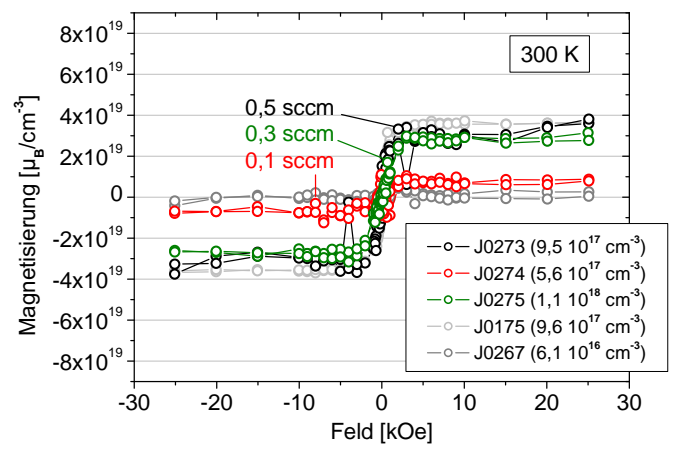

\begin{abstract}
Abbildung 4.6:
Vergleich der Hysteresen von Sauerstoff-Co-dotiertem GaN:Gd bei 300 K. Der Sauerstofffluß während des Wachstums ist in der entsprechenden Farbe zur Kurve angegeben. Die grauen Kurven sind die Schicht J0175 aus Abbildung 4.4(a) und J0267. J0175 wurde ca. 4 Jahre vor den Sauerstoffco-dotierten Schichten hergestellt, J0267 ist eine zeitnah hergestellte GaN:Gd-Schicht.
\end{abstract}

zu gleich starke Sättigungsmagnetisierung zu finden, welche mit der Magnetisierung der J0175 vergleichbar ist. Für die Probe mit geringstem Sauerstoffangebot ist die Sättigungsmagnetisierung deutlich reduziert (J0274). Die zeitnah zu den Sauerstoff-codotierten Schichten hergestellte Schicht J0267 zeigt kein ferromagnetisches Verhalten. Dieses deutet auf den Einfluß des Sauerstoffes auf die magnetischen Eigenschaften der GaN:Gd-Schichten hin.

\title{
4.5 Allgemeine Trends
}

In Abbildung 4.7(a) sind die Trends der Proben mit magnetischer Ordnung zusammengefaßst. Es sind alle in den vorherigen Abschnitten diskutierten Proben enthalten. Aufgetragen wurde die absolute Sättigungsmagnetisierung gegen die Gd-Konzentration. Geschlossene Symbole sind Proben der Serie 1 und offene Symbole Proben der Serie 2. Gut sichtbar ist hier der Trend der steigenden Magnetisierung mit der Gd-Konzentration, welche aber wieder für hohe Gd-Konzentrationen abfällt. In beiden Serien fällt auf, daß für die Schichten mit der jeweils höchsten Sättigungsmagnetisierung (J0175, J0172 und J0265, J0264) die jeweils höher mit Gd-dotierte eine geringere Magnetisierung aufweist. Für Serie 1 ist dieser Unterschied im Rahmen des Fehlers, aber für Serie 2 ist die Änderung signifikant. Schichten mit noch höheren Gd-Konzentrationen zeigen in beiden Serien keine Magnetisierung. Die Magnetisierung der Wasserstoff-co-dotierten Schichten ist im allgemeinen gegenüber denen der nicht co-dotierten reduziert. Insgesamt ist das Verhalten dieser Schichten im Einklang mit den Ergebnissen von Hite et al. [31]. Dort wird eine maximale Sättigungsmagnetisierung für eine Gd-Zellentemperatur von $1050^{\circ} \mathrm{C}$ ge- 


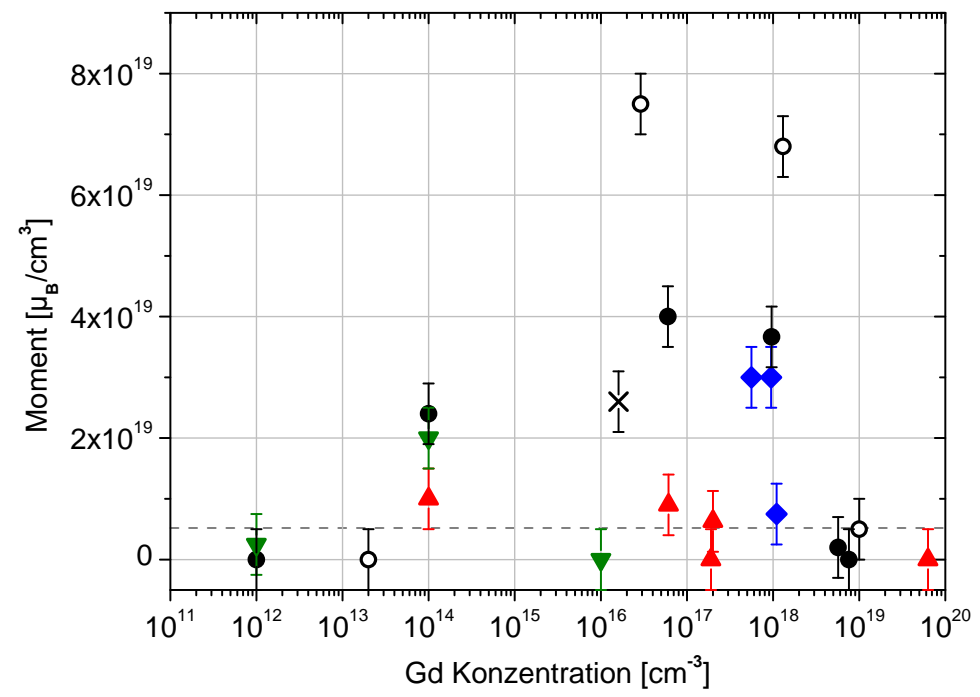

(a) absolute Sättigungsmagnetisierung pro Volumen

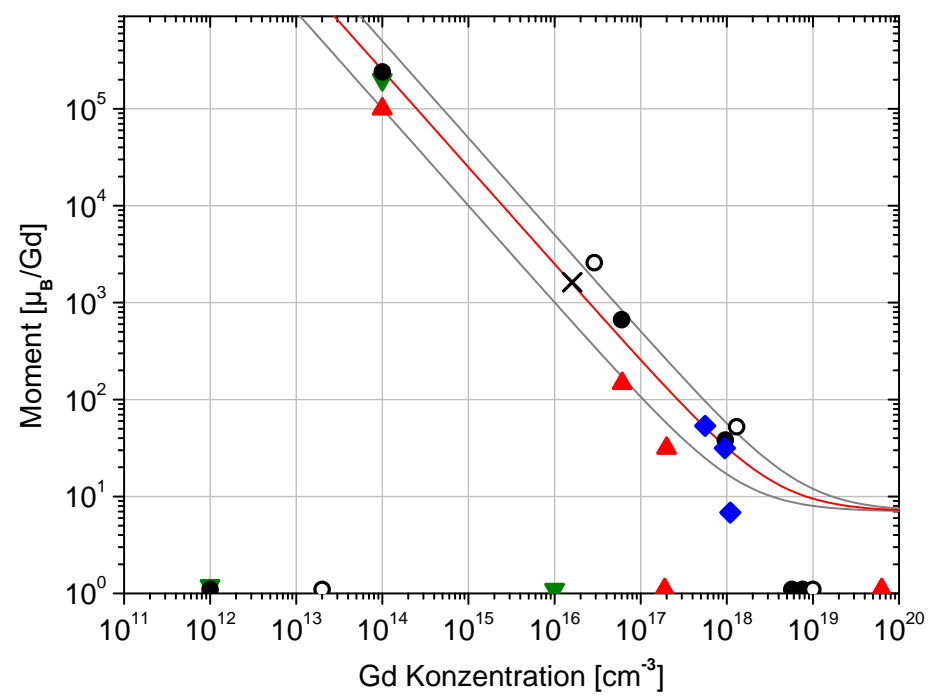

(b) effektive Sättigungsmagnetisierung pro Gd

\section{Abbildung 4.7:}

Übersicht über die absoluten und effektiven Sättigungsmagnetisierungen. Schwarze Kreise stehen für nicht co-dotiertes GaN:Gd, das schwarze Kreuz ist die auf SiC hergestellte Probe J0188, rote Dreiecke für Wasserstoff co-dotiertes GaN:Gd, grüne Dreiecke für Silizium co-dotiertes GaN:Gd und blaue Rauten für Sauerstoff co-dotiertes GaN:Gd. Geschlossene Symbole sind Proben der Serie 1, offene der Serie 2. (a) zeigt die absoluten Magnetisierungen der Schichten in $\mu_{\mathrm{B}} / \mathrm{cm}^{3}$, (b) zeigt die effektive Magnetisierung pro Gd Atom in $\mu_{\mathrm{B}}$. Die Kurven sind nach Formel $1.1 \mathrm{mit}$ $\mathrm{m}_{\text {Def }} \cdot \mathrm{c}_{\text {Def }}=10^{19}, 2,5 \cdot 10^{19}$ und $5 \cdot 10^{19} \mu_{\mathrm{B}} / \mathrm{cm}^{3}$ berechnet. 
funden. Diese Zellentemperatur würde im Rahmen dieser Arbeit einer Gd-Konzentration von ca. $3 \cdot 10^{17} \mathrm{~cm}^{-3}$ entsprechen und liegt damit in der gleichen Größenordnung wie der hier gefundene Wert von ca. $5 \cdot 10^{17} \mathrm{~cm}^{-3}$ für die stärksten Magnetisierungen. Sauerstoffco-dotiertes GaN:Gd zeigt wiederum Sättigungsmagnetisierungen, welche mit denen der nicht co-dotierten GaN:Gd-Schichten der Serie 1 vergleichbar sind (blaue Rauten). Die Abhängigkeit der Sättigungsmagnetisierung vom Sauerstoffangebot spiegelt sich hier in der Probe J0274 mit deutlich niedrigerer Sättigungsmagnetisierung wider. Eine starke Steigerung der Magnetisierung durch Si-co-Dotierung kann nicht bestätigt werden [31, 18]. Das Bild des Elektronen-induzierten Magnetismus, wie von Dalpian und Wei vorgeschlagen, ist nicht im Einklang mit den hier gezeigten Daten [36].

Die Betrachtung der mit Wasserstoff-co-dotierten Schichten bietet einen interessanten Hinweis auf die Art des Defektes, welcher vermutlich mit für die magnetischen Eigenschaften in GaN:Gd verantwortlich ist. Gd-dotiertes GaN:Gd ist hochohmig [17, 18, 25], während undotiertes GaN zu n-Leitfähigkeit neigt. Die Vermutung, daß ein durch das Gd-induzierter Akzeptor die vorherrschende Ladungsträgersorte kompensiert ist hier naheliegend. Mit der Dotierung von Wasserstoff wird die Sättigungsmagnetisierung von GaN:Gd reduziert. Wasserstoff passiviert Akzeptoren in GaN durch Komplexbildung und ist ein amphoterer Defekt $\left(\mathrm{H}_{\mathrm{i}}\right)$ [38]. Bei einer Lage der Fermienergie in der Mitte der Bandlücke wird durch Wasserstoff eine Reduktion der Löcherkonzentration erwartet. Diese beiden Faktoren unterstützen den Gedanken, daß der für den Magnetismus aktive Defekt ein vom Gd induzierter Akzeptor ist.

\subsection{Effektive Magnetisierung}

In Abschnitt 1.2.2 wurden bereits die effektiven Magnetisierungen ausgewählter Schichten mit denen von Dhar et al. verglichen. Es konnte festgestellt werden, daß die kolossalen Momente gut reproduziert werden und daß sich diese sogar für noch geringere Gd-Konzentrationen als bei Dhar et al. zu finden sind [17, 19]. Abbildung 4.7(b) zeigt die zu Abbildung 4.7(a) gehörige Auftragung der effektiven Sättigungsmagnetisierung in $\mu_{\mathrm{B}} / \mathrm{Gd}$. Schichten mit einer effektiven Magentisierung unterhalb von $1 \mu_{\mathrm{B}} / \mathrm{cm}^{3}$ sind der Übersicht halber an den unteren Rand der Abbildung gezeichnet. Die Kuren sind wie in Abschnitt 1.2.2 nach Formel 1.1, mit $\mathrm{m}_{\text {Def }} \cdot \mathrm{c}_{\text {Def }}=10^{19}, 2,5 \cdot 10^{19}$ und $5 \cdot 10^{19} \mu_{\mathrm{B}} / \mathrm{cm}^{3}$ gezeichnet und spiegeln die Theorie wider, daß die magnetischen Momenten nicht vom Gd getragen werden, sondern hauptsächlich von einem Defekt mit konstanter Konzentration (Abschnitt 1.2.2).

Im folgenden wird ein Defekt mit einem magnetischen Moment von einem $\mu_{\mathrm{B}}$ angenommen und damit wie in Abschnitt 1.2.2 eine Abschätzung der für den Magnetismus aktiven Defektkonzentration gemacht. Bei Defekten mit höheren Momenten würden die 


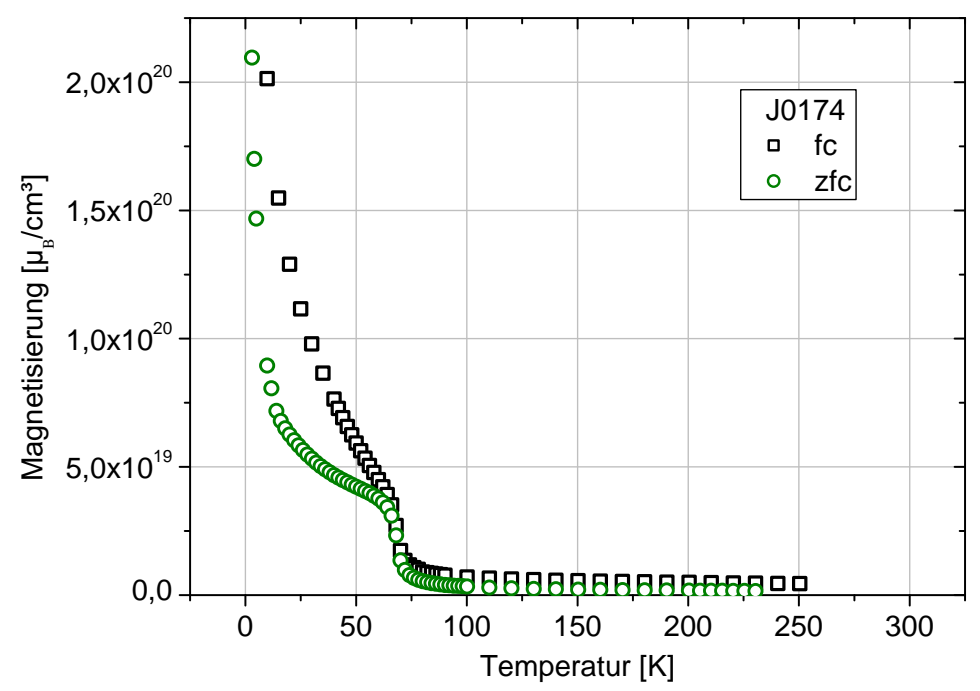

\section{Abbildung 4.8:}

Temperaturabhängige Magnetisierung der Probe J0174 bei einem Meßfeld von 100 Oe. Die Schicht enthält $1,6 \cdot 10^{20} \mathrm{~cm}^{-3} \mathrm{Gd}$. XRD zeigt GdN Ausscheidungen. Bei ca. $70 \mathrm{~K}$ ist ein magnetischer Übergang zu erkennen, welcher GdN als Ausscheidung bestätigt. Das Meßfeld betrug 100 Oe.

genannten Konzentrationen entsprechend nach unten skalieren. Die Magnetisierungen von nicht co-dotiertem, Si-co-dotiertem und O-co-dotiertem GaN:Gd der Serie 1 werden gut durch eine Defektkonzentration von ca. $2,5 \cdot 10^{19} \mathrm{~cm}^{-3}$ beschrieben (rote Kurve). Auch eine auf $\mathrm{SiC}$ hergestellte Schicht (schwarzes Kreuz) paßt gut in dieses Bild. Bei H-co-dotiertem GaN:Gd ist die Defektkonzentration verringert und beläuft sich nur auf ca. $10^{19} \mathrm{~cm}^{-3}$. Diese Tatsache ist in gutem Einklang mit den Ergebnissen aus dem vorangehenden Abschnitt, wo eine Reduktion des für den Magnetismus aktiven Defekts diskutiert wurde. Die Frage, welche Defekte hinter diesem Verhalten stecken können, wird weiter in Kapitel 5 diskutiert.

\subsection{Ausscheidungen}

Für die höchste Gd-Konzentration von 1, $6 \cdot 10^{20} \mathrm{~cm}^{-3}$ (J0174) weichen die magnetischen Eigenschaften deutlich von den bisher gezeigten ab. Abbildung 4.8 zeigt die temperaturabhängigen Messungen der Magnetisierung. Es ist ein deutlicher magnetischer Übergang zwischen 60 und $70 \mathrm{~K}$ zu erkennen. Bei Raumtemperatur ist kein hysteretisches Verhalten zu beobachten. Unterhalb der Übergangstemperatur ist eine deutliche Hysterese mit einem Koerzitivfeld von 60 Oe vorhanden.

Der magnetische Übergang bei ca. $70 \mathrm{~K}$ deutet auf die Bildung einer zweiten Phase 


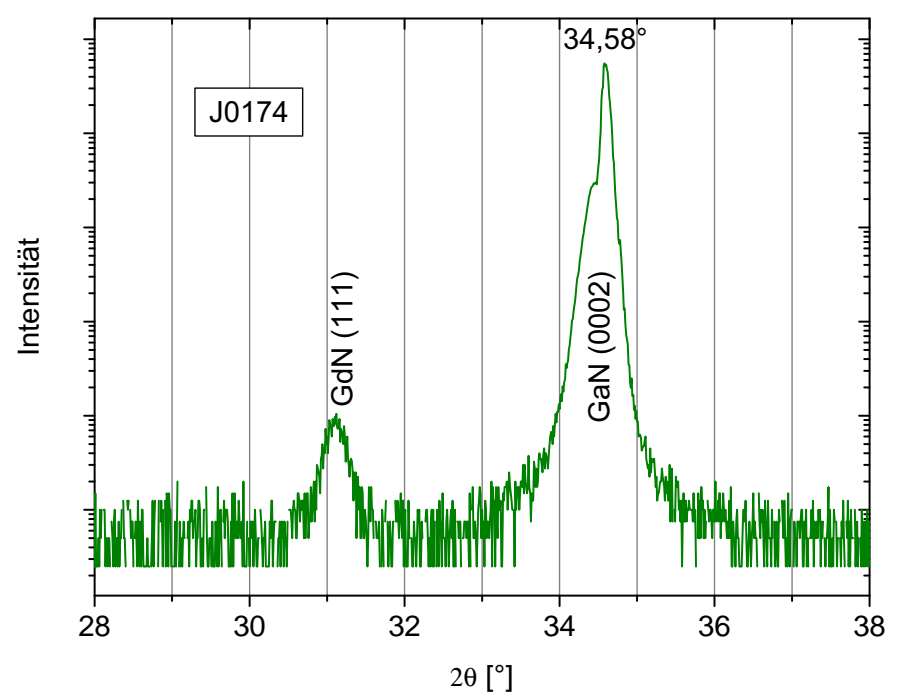

\begin{abstract}
Abbildung 4.9:
XRD $\theta-2 \theta$-Messung der Probe J0174. Der Reflex bei $34,58^{\circ}$ ist das GaN-Template auf Saphir, die Schulter zu kleineren Winkeln entsteht durch die verspannte GaN:Gd-Schicht. Der Reflex bei $31,1^{\circ}$ ist kubischen GdN-Ausscheidungen zuzuweisen.
\end{abstract}

aus kubischem GdN hin. XRD Messungen bestätigen diese Vermutung (Abbildung 4.9). Bei einem Winkel von $2 \theta=31,1^{\circ}$ ist der (111) Reflex von kubischem GdN zu sehen. Die Schulter zu niedrigeren Winkeln am (0002) GaN Reflex läßt auf eine Vergrößerung der c-Gitterkonstante gegenüber dem GaN Template schließen. Auswertung des Signals nach der Scherrerformel liefert eine Größe der Auscheidungen von ca. $30 \mathrm{~nm}$. Das Signal bei $34,58^{\circ}$ ist dem GaN Template zuzuordnen. Kubisches GdN ist ferromagnetisch und die Curietemperatur wird zwischen $58 \mathrm{~K}$ und $69 \mathrm{~K}$ bestimmt [58, 59]. Das hysteretische Verhalten unterhalb der Übergangstemperatur (nicht gezeigt) ist auf geblockten Superparamagnetismus zurückzuführen [11].

Das Auftreten dieser ferromagnetischen zweiten Phase läßt die Vermutung zu, die magnetischen Eigenschaften von GaN:Gd, wie z.B. auch bei GaN:Fe [15], sind auf die Bildung von Nanomagneten in der GaN-Matrix zurückzuführen. Allerdings ist die Bildung von GdN-Ausscheidungen nicht in der Lage die kolossalen Momente oder die kollektiven Effekte bei Raumtemperatur und darüber zu erklären, da deren Curietemperatur bei ca. $70 \mathrm{~K}$ liegt. In der Arbeit von Dhar et al. ist ein vergleichbarer magnetischer Übergang für alle GaN:Gd-Schichten zu finden [17]. Die Tatsache, daß dieser Übergang im Rahmen dieser Arbeit nur für die Probe mit höchster Gd-Konzentration gefunden wurde und mit dem Erscheinen der Hysterese bei Raumteperatur verschwindet, schließt die GdN-Ausscheidungen als Grund für den Ferromagnetismus weiter aus. 


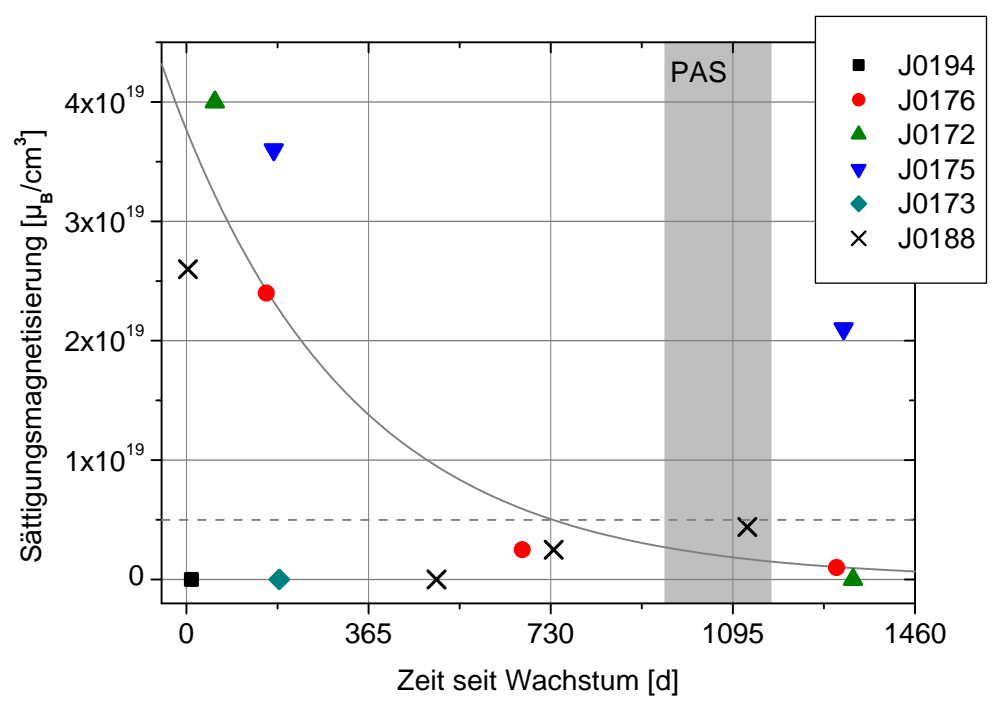

\begin{abstract}
Abbildung 4.10:
Zeitabhängigkeit der Sättigunsmagnetisierung bei $300 \mathrm{~K}$ ausgesuchter Proben der Serie 1. Die gestrichelte Linie ist die geschätzte Artefaktgrenze des SQUID, die exponentielle Kurve ist als Orientierungshilfe gezeigt. Alle Proben außer der J0175 verlieren nach ca. 1 Jahr ihre Magnetisierung. Der grau hinterlegte Bereich markiert den Zeitraum der PAS-Messungen aus Abschnitt 5.3.
\end{abstract}

\title{
4.8 Probenalterung
}

Die Probenalterung im Bezug auf die magnetischen Eigenschaften wurde anhand von SQUID-Messungen in einem Zeitraum von ca. 4 Jahren untersucht. Abbildung 4.10 zeigt die Sättigungsmagnetisierung nicht co-dotierter GaN:Gd-Schichten der Serie 1 gegen die Zeit seit dem Wachstum der Probe aufgetragen. Die unterschiedlichen Proben sind farblich codiert. Die horizontale Linie spiegelt die Artefaktgrenze des SQUID wider. Der eingezeichnete exponentielle Abfall dient als Orientierung und hat keine weitere physikalische Bedeutung.

Für fast alle als ferromagnetisch eingestuften Schichten ist ein eindeutiger Abfall der Sättigungsmagnetisierung auf Null innerhalb der ersten 1,5 Jahre festzustellen (J0172, J0176, J0188). Zum Vergleich sind auch die beiden als nicht ferromagnetisch eingestuften Schichten J0173 und J0194 gezeigt. Für diese Proben kann ausgeschlossen werden, daß eine zu späte Messung eine Verfälschung der magnetischen Daten verursacht hat. Als Halbwertszeit der Magnetisierung kann grob ein Jahr angesetzt werden, eine genaue Bestimmung lassen die vorliegenden Daten aber nicht zu.

Probe J0175 zeigt ein abweichendes Verhalten. Eine Messung ca. 3 Jahre nach der Herstellung zeigt eine reduzierte, aber deutliche Magnetisierung. Der Grund für dieses unterschiedliche Verhalten ist unbekannt, es ist aber festzuhalten, daß die Probe J0175 
auch bei den PAS-Messungen in Abschnitt 5.3 abweichende Ergebnisse zeigt.

Deutlich wird bei dieser Betrachtung des zeitlichen Verlaufes der Sättigungsmagnetisierung, daß der für das ferromagnetische Verhalten der Schichten verantwortliche Parameter nicht stabil ist. Mitra und Lambrecht errechnen eine starke Tendenz der Bildung von $\mathrm{Gd}_{\mathrm{Ga}}-\mathrm{O}_{\mathrm{i}}$ Komplexen unter Wachstumsbedingungen. Für Umgebungsbedingungen nach dem Wachstum kann aber keine starke Bindung zwischen dem Gd und dem O gefunden werden, was für die Instabilität des Komplexes spricht. Zusätzlich wird eine ferromagnetische Wechselwirkung zwischen Sauerstoffzwischengitteratomen vorhergesagt. Nimmt man das Ergebnis von Mitra und Lambrecht, die Abhängigkeit der Sättigungsmagnetisierung vom Sauerstoffangebot und die Alterung der GaN:Gd Schichten zusammen, so kann man Sauerstoff als den verantwortlichen Defekt vermuten. Auch die von Dhar et al. und Hite et al. gefundenen hohen Sauerstoffkonzentrationen, welche in den Bereich der in Abschnitt 4.5 abgeschätzten Defektkonzentration von ca. $2,5 \cdot 10^{19} \mathrm{~cm}^{-3}$ fallen, deuten auf Sauerstoff hin. Diese Überlegung ist auch mit dem in Abschnitt 4.5 gewonnenen Hinweis auf einen Akzeptor in Einklang, da für $\mathrm{O}_{\mathrm{i}}$ und $\mathrm{Gd}_{\mathrm{Ga}}-\mathrm{O}_{\mathrm{i}}$ Akzeptorverhalten vorhergesagt wird $[49,40]$.

\section{Zusammenfassung}

In diesem Kapitel wurden die magnetischen Eigenschaften von GaN:Gd und die Auswirkungen von co-Dotierungen auf diese diskutiert. Die Übersicht über eine große Anzahl von Proben zeigt eine schlechte Reproduzierbarkeit der magnetischen Eigenschaften von GaN:Gd. Das Auftreten von kollektiven Phänomenen ist dabei aber nicht willkürlich über die verschiedenen Proben verteilt, sondern ändert sich phasenweise. Es wurde auf einen sich systematisch verändernden unberücksichtigten Parameter geschlossen. Nicht co-dotiertes GaN:Gd zeigt eine steigende Sättigungsmagnetisierung mit steigender Gd-Konzentration, wobei Schichten mit hohen Gd-Konzentrationen kein ferromagnetisches Verhalten mehr zeigen. Si-co-Dotierung führt zu einem Verschwinden der Magnetisierung schon zu geringeren Gd-Konzentrationen als für nicht co-dotiertes Material. Wasserstoff-co-Dotierung reduziert die gemessenen Momente. Hinweise auf einen Akzeptor als den für den Magnetismus aktiven Defekt konnten gefunden werden. Für Sauerstoff als co-Dotierung wurde eine Abhängigkeit vom Sauerstoffangebot festgestellt. Die von Dhar et al. gefundenen kolossalen Momente werden gut reproduziert und es kann eine Defektkonzentration von ca. 2,5 $\cdot 10^{19} \mathrm{~cm}^{-3}$ abgeschätzt werden. Für Wasserstoffco-dotierte Proben ist diese Konzentration auf ca. $10^{19} \mathrm{~cm}^{-3}$ verringert. Für hohe GdKonzentrationen wird die Bildung einer GdN Phase festgestellt, welche aber als Verantwortlicher für die Raumtemperaturmagnetisierung ausgeschlossen werden kann. Die Proben unterliegen einem Alterungsprozess der magnetischen Eigenschaften, welcher 
den Verlust der Magnetisierung innerhalb eines Jahres zur Folge hat.

Alles in allem deutet das magnetische Verhalten auf den Einfluß von Akzeptoren hin und die gefundenen Eigenschaften können nicht auf die alleinige Anwesenheit von Gd in der GaN-Matrix zurückgeführt werden. Dabei fällt besonders Sauerstoff ins Auge, kann aber noch nicht als der verantwortliche Defekt nachgewiesen werden. Ungeklärt bleibt bis jetzt auch die Frage, warum der verantwortliche Defekt nur in Anwesenheit von Gd aktiv wird, aber die Konzentration unabhängig von der Gd-Konzentration scheint. 


\section{Kapitel 5}

\section{Strukturelle Eigenschaften und Korrelationen zum Magnetismus}

In diesem Kapitel werden die strukturellen Eigenschaften von Gd-dotiertem GaN untersucht und Korrelationen mit den magentischen Eigenschaften diskutiert. Der erste Abschnitt dieses Kapitels geht auf das Thema der Kristallqualität ein und untersucht die Möglichkeit, daß Ausscheidungen die magnetischen Eigenschaften von GaN:Gd erzeugen anhand von XRD und TEM. Abschnitt 5.2 beschäftigt sich mit der atomaren Umgebung des Gd in der GaN-Matrix. Es werden Ergebnisse aus Röntgenabsoption (XANES) und paramagnetischer Resonanz (EPR) gezeigt. Gefolgt davon wird in Abschnitt 5.3 die Galliumleerstelle näher mit Hilfe der Positronenvernichtung (PAS) untersucht. Abschnitt 5.4 zeigt optische Eigenschaften von GaN:Gd und abschliesend wird das Kapitel zusammengefaßst. 


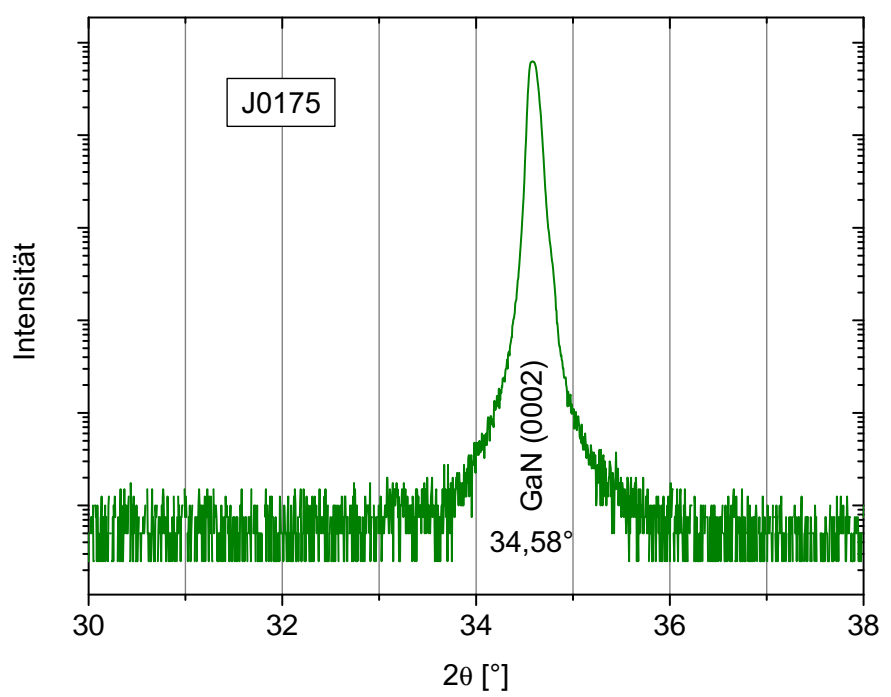

\begin{abstract}
Abbildung 5.1:
XRD $\theta-2 \theta-M e s s u n g$ der Schicht J0175. Der GaN (0002)-Reflex bei $34,58^{\circ}$ ist nicht vom unterliegenden GaN-Template auf Saphir zu unterscheiden und es sind keine Anzeichen einer zweiten Phase zu erkennen. Der (111)-Reflex von kubischem GdN würde bei $31,1^{\circ}$ erscheinen (vgl. Abbildung 4.9).
\end{abstract}

\title{
5.1 Kristallqualität
}

Die Kristallqualität wurde mit Hilfe von XRD-Messungen charakterisiert. In den $\theta$ $2 \theta$ Messungen sind keine Anzeichen für Ausscheidungen zu erkennen (Ausnahme: J0174, $\mathrm{c}_{\mathrm{Gd}}=1,6 \cdot 10^{20} \mathrm{~cm}^{-3}$, Abbildung 4.9). Abbildung 5.1 zeigt die Messung der Probe J0175 mit einer Gd-Konzentration von 9,6 $10^{17} \mathrm{~cm}^{-3}$. Der Vergleich mit Probe J0174 (Abbildung 4.9) zeigt deutliche Unterschiede. Das GdN-(111)-Signal bei $31,1^{\circ}$ und die Schulter zu kleineren Winkeln am Signal des GaN-Templates bei $34,58^{\circ}$ sind nicht vorhanden. Die Halbwertsbreiten (FWHM) der rocking curves aller Schichten (inklusive undotiertem GaN) liegen zwischen 400 und 470 arcsec für den (0002)-Reflex und zwischen 400 und 630 arcsec für die (1012)- und (112̄2)-Reflexe. Diese Werte zeigen eine gute Kristallinität der GaN:Gd-Schichten und bestätigen, daß die Dotierung mit Gd nur geringe Einflüsse auf das Wachstum der GaN-Matrix hat. Ausnahme ist die Probe J0188 ((0002): $524 \operatorname{arcsec},(10 \overline{1} 2): 1300 \operatorname{arcsec},(11 \overline{2} 2): 1962$ arcsec). Der Grund für die höheren Werte ist auf das SiC-Template zurückzuführen.

Weiter kann die allgemein gute Qualität der Schicht anhand von TEM-Aufnahmen bestätigt werden. Abbildung 5.2 zeigt hochauflösende Aufnahmen der Grenzfläche der GaN:Gd-Schicht J0188 zum SiC-Substrat. Die Grenzfläche ist von guter Qualität und es sind nur wenige Defekte zu erkennen. Das GaN-Wachstum auf SiC liefert in der 


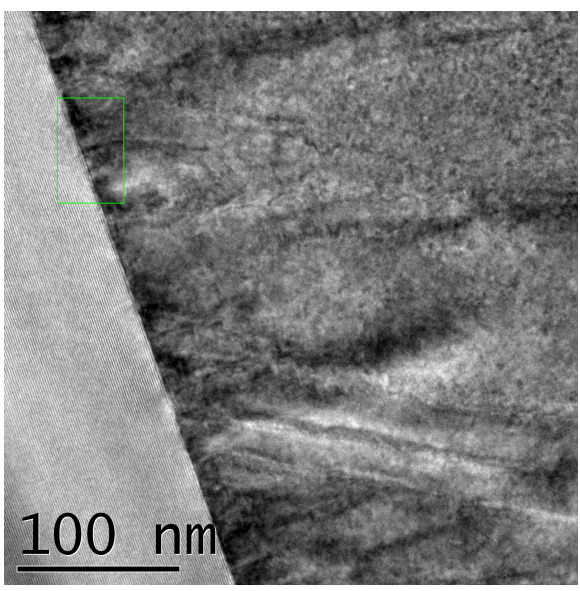

(a)

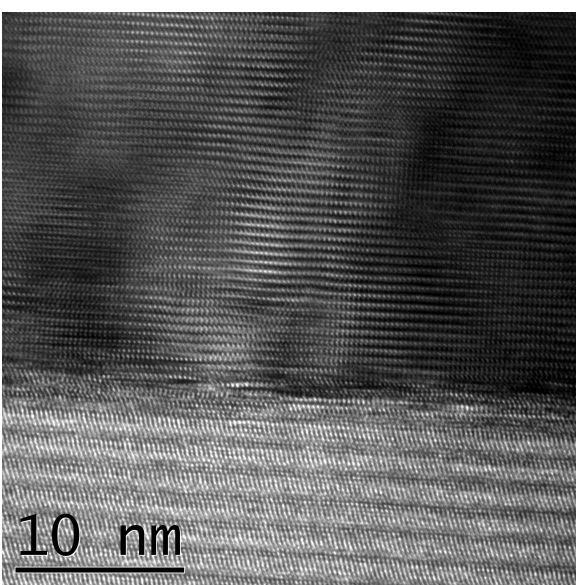

(b)

\section{Abbildung 5.2:}

Hochauflösende TEM Aufnahmen der Probe J0188. Links/unten ist das SiC-Substrat zu sehen, darüber die MBE-GaN:Gd-Schicht.

Regel Schichten mit schlechterer Qualität als auf GaN-Templates, was auch durch die XRD-Messungen bestätigt wird. In der GaN:Gd-Schicht sind keine Ausscheidungen zu erkennen. Selbst wenn nanoskalige Partikel nicht ausgeschlossen werden können, so kann doch ein Einfluß von wie in Probe J0174 gefundenen Ausscheidungen von mehreren $10 \mathrm{~nm}$ ausgeschlossen werden.

\subsection{Einbauort und Ladungszustand des Gd}

Gema Martínez-Criado hat XRF- und XANES-Messungen an GaN:Gd-Schichten mit Gd-Konzentrationen von $10^{16}-10^{18} \mathrm{~cm}^{-3}$ durchgeführt. Die Messungen wurden an der ID22 an der European Synchrotron Radiation Facility (ESRF) durchgeführt [21]. Die EPR-Messungen wurden von Prof. Gehlhoff an der TU Berlin durchgeführt.

\subsubsection{Röntgenfluoreszens und -absorption}

Das XRF Spektrum in Abbildung 5.3(a) zeigt Fluoreszenzen von Ga, Gd, Ca, S, Si, Ar und Fe. Es ist vorab nicht auszuschließen, daß die Signale aus der GaN:Gd-Schicht stammen. Bei einer Eindringtiefe der Mehtode von ca. $8 \mu \mathrm{m}$, besteht aber die Möglichkeit, daß die Signale aus dem Substrat stammen. Das Ar Signal ist auf die Umgebungsluft zurückzuführen. In Abschnitt 3.8 konnte bereits eine Fe-Verunreinigung der Schichten weitestgehend ausgeschlossen werden. Auch wenn geringe Konzentrationen von Fe möglich sind, können sie als vernachlässigbar gegenüber der Gd-Konzentration angesehen 


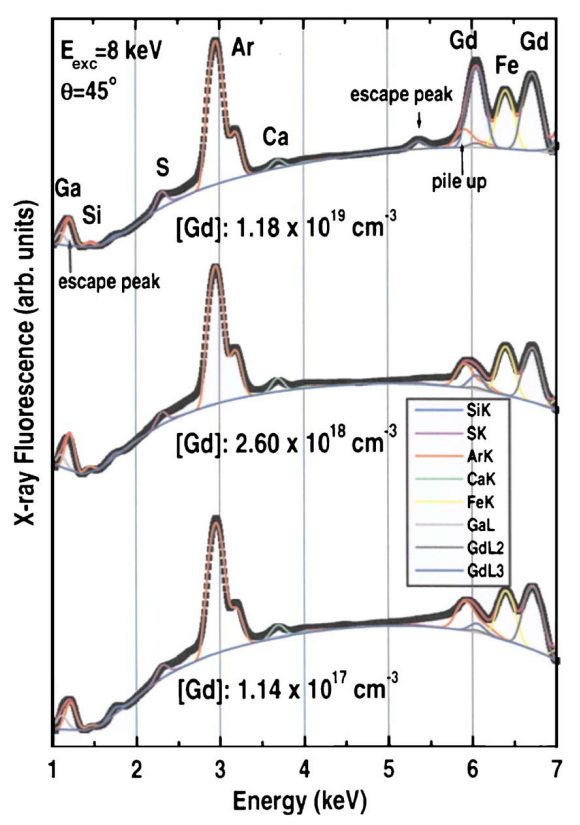

(a) XRF

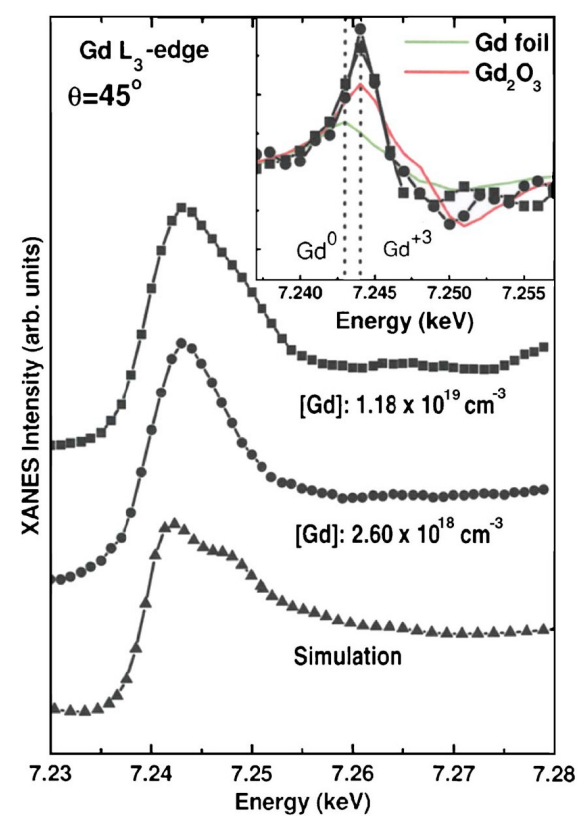

(b) XANES

\section{Abbildung 5.3:}

Röntgenfluoreszenz- (XRF) und Röntgenabsorptionsmessungen (XANES) an den Proben J0172, J0175 und J0176. Alle drei Proben sind zum Zeitpunkt der Messung ferromagnetisch bei Raumtemperatur. Die genannten Konzentrationen sind aufgrund einer anfänglichen Ungenauigkeit in der Auswertung der SIMS Daten ca. eine Größenordnung zu hoch angegeben (Abschnitt 3.2). Abbildungen aus [21].

werden. Die Homogenität der Proben wurde auf Größe des Strahldurchmessers untersucht. Beim Rastern der Probe mit einem Strahlquerschnitt von 1,5 x 3,5 $\mu^{2}$ konnten keine Intensitätsschwankungen im XRF-Spektrum gefunden werden. Dieses läßt den Schluß zu, daß das Gd auf dieser Skala homogen in der Schicht eingebaut ist und steht damit auch in Einklang mit den SIMS Messungen aus Abschnitt 3.5.

Abbildung 5.3(b) zeigt die XANES-Daten an der Gd L $\mathrm{L}_{3}$ Kante der beiden GaN:GdSchichten mit höherer Gd-Konzentration zusammen mit einer Simulation für Gd auf einem Ga-Platz in wz-GaN. Für eine Gd-Konzentration von ca. $10^{16} \mathrm{~cm}^{-3}$ konnte keine $\mathrm{Gd} \mathrm{L}_{3}$ Kante beobachtet werden. In den Messungen wird keine Veränderung der Absorptionskante für die verschiedenen Gd-Konzentrationen gefunden und die Übereinstimmung mit der Simulation ist gut. Im Einsatz der Abbildung ist die erste Ableitung der Messung bei hoher Gd-Konzentration gezeigt und zum Vergleich die Daten von Referenzmessungen an Gd-Folie und $\mathrm{Gd}_{2} \mathrm{O}_{3}$, also einem System mit $\mathrm{Gd}^{0}$ - und einem mit $\mathrm{Gd}^{3+}$-Zustand. Es ist zu erkennen, daß die Messungen in GaN:Gd und an der $\mathrm{Gd}^{3+}$ 


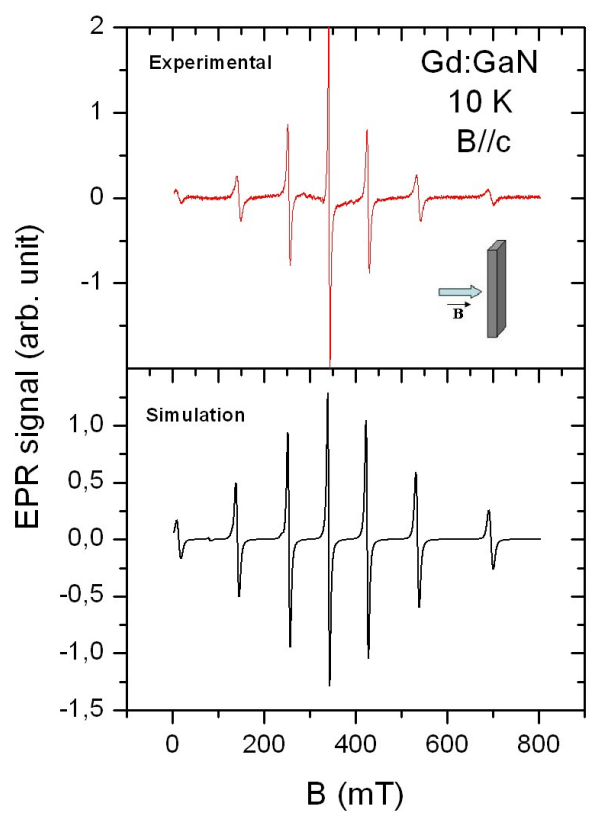

(a) parallel

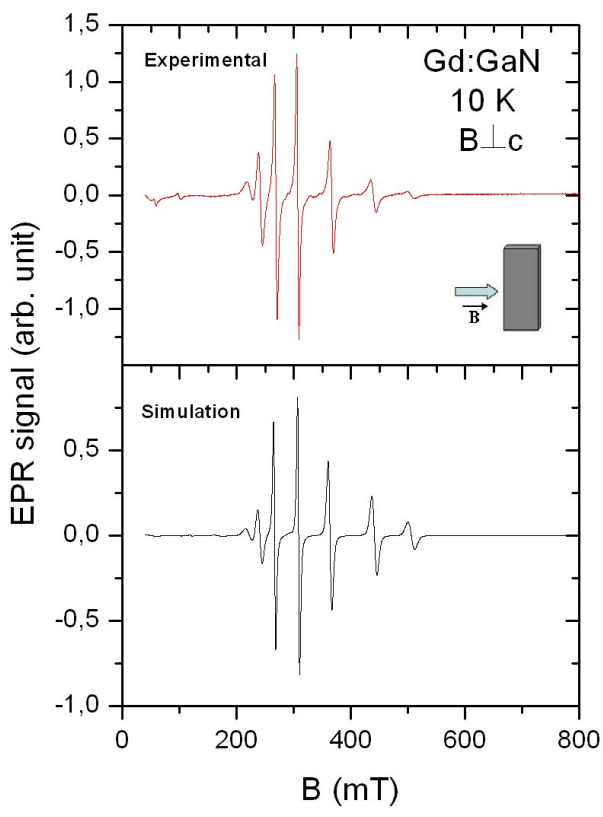

(b) senkrecht

\section{Abbildung 5.4:}

EPR-Messungen an der Schicht J0252. Die Messung stimmt gut mit der Simulation überein und läßt auf eine ungestörte GaN-Matrix im Umfeld des Gd-Atoms schließen.

Referenz übereinstimmen. Damit wird gezeigt, daß Gd in GaN auf einem Ga-Platz eingebaut wird und im Ladungszustand $3+$ vorliegt.

\subsubsection{EPR}

Weitere Informationen über die strukturelle Umgebung von Gd in GaN kann durch EPR-Messungen der Gd-Resonanzen in GaN gewonnen werden. Die Messungen wurden von Prof. Gehlhoff an der TU Berlin durchgeführt und sind in der Diplomarbeit von Ole Hitzemann diskutiert [60]. Hier sollen nur die wichtigsten Ergebnisse zusammengefaßt werden. Probe J0252 ist mit einer Gd-Konzentration von $1,1 \cdot 10^{18} \mathrm{~cm}^{-3}$ und einer Schichtdicke von 1,8 $\mu \mathrm{m}$ gut für EPR-Messungen geeignet und wurde im X- und Q-Band $(9,5$ und $34 \mathrm{GHz})$ untersucht. Abbildung 5.4 zeigt die EPR-Spektren aus den $\mathrm{X}$-Band-Messungen und die zugehörigen Simulationen. Die gezeigte Simulation spiegelt $\mathrm{Gd}^{3+}$ auf einem Ga-Platz in einem sonst ungestörten wz-GaN Kristall wider. Diese Simulation zeigte die besten Übereinstimmung mit den experimentellen Spektren und es kann gefolgert werden, daß die GaN-Matrix in der Umgebung des Gd-Atoms keine Veränderungen gegenüber ungestörtem GaN aufweist. Insbesondere kann so eine An- 
häufung von $\mathrm{V}_{\mathrm{Ga}}$ oder $\mathrm{V}_{\mathrm{N}}$ ausgeschlossen werden. Der von Gohda et al. oder Dev et al. vermutete Grund für die kolossalen Momente, eine Anhäufung von Galliumleerstellen um das Gd-Atom, kann deshalb ausgeschlossen werden [35, 46].

\subsection{Positronenvernichtung}

In vielen theoretischen und experimentellen Arbeiten wird über die Rolle der Galliumleerstelle als ein wichtiger Defekt für die magnetische Ordnung in GaN:Gd diskutiert. Im vorangehenden Abschnitt konnte mittels EPR gezeigt werden, daß keine Anhäufungen von Vakanzen im direkten Umfeld von Gd zu finden sind. Um die Rolle der $\mathrm{V}_{\mathrm{Ga}}$ weiter zu untersuchen, wurden magnetische und nicht-magnetische Schichten in Kooperation mit der Aalto Universität Helsinki mit PAS charakterisiert. Die Methode ist insbesondere für Leerstellendefekte, aber auch für Punktdefekte ohne Leervolumen im Kristall (negativ geladen oder neutral) sensitiv. Eine kurze Einführung in die Methode findet sich in Anhang C. Die Messungen wurden von Christian Rauch durchgeführt.

Die PAS-Messungen wurden ca. 2 Jahre nach der Herstellung der Schichten durchgeführt (grau hinterlegter Bereich in Abbildung 4.10). Da innerhalb dieser Zeit fast alle Schichten einen Verlust der Magnetisierung aufweisen, kann im folgenden nur Probe J0175 als ferromagnetische Probe angesehen werden.

Abbildung 5.5(a) zeigt den SW-Plot verschiedener GaN-Schichten. Als rote Kreise sind die SW-Parameter für Leerstellen-freies GaN und für die Galliumleerstelle eingetragen. Auf der Geraden zwischen diesen beiden Punkten wird GaN mit einzelnen, undekorierten Galliumleerstellen erwartet. Für Leerstellenkonzentrationen von ca. $10^{16}$ bis $10^{19} \mathrm{~cm}^{-3}$ liegen die SW-Parameter zwischen diesen Punkten, höhere oder niedrigere Konzentrationen fallen mit den Punkten für die Leerstelle oder den GaN-Volumenkristall zusammen. Dekorierte Leerstellen oder Komplexe mit großem Leervolumen im Kristall werden im Bereich rechts und oberhalb der roten Gerade erwartet ([61] und Referenzen darin).

Für fast alle der untersuchten GaN:Gd-Schichten und die GaN-Referenzen werden hohe Konzentrationen an Defekten mit Leervolumen im Kristall gefunden. Die Konzentrationen liegen im Bereich zwischen $10^{15}-10^{17} \mathrm{~cm}^{-3}$. Es ist keine direkte Korrelation der Gd-Konzentration oder der Magnetisierung mit den S- oder W-Parametern zu erkennen. Allerdings wird die Probe J0175, welche als einzige ferromagnetisch während der PAS-Messungen war, bei S- und W-Parametern gefunden, welche darauf hinweisen, daß keine Defekte mit Leervolumen in nennenswerten Konzentrationen vorliegen. $\mathrm{Zu}$ erkennen ist dieses in Abbildung 5.5(a) an dem nahezu mit der GaN-Referenz zusammenfallendem Punkt ${ }^{1}$ für Probe J0175. Mit diesem Ergebnis kann die von Gohda et al.

\footnotetext{
${ }^{1}$ Bei höheren S- und niedrigeren W-Parametern liegende Punkte lassen auf eine deutliche Veränderung
} 


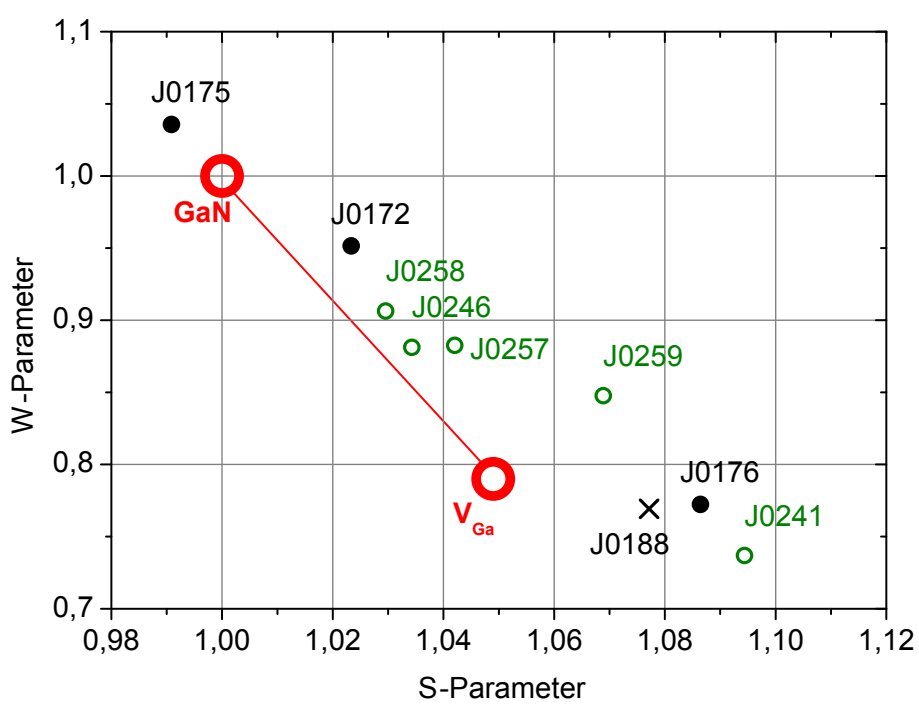

(a) S(W) Plot verschiedener GaN:Gd Schichten

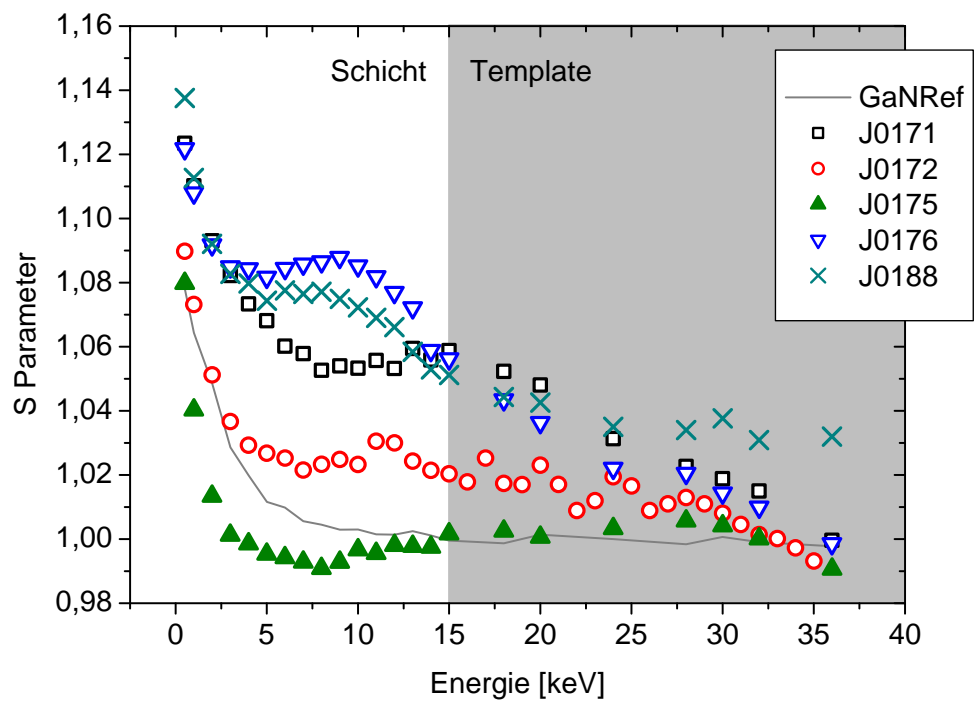

(b) Tiefenprofil des S Parameters

\section{Abbildung 5.5:}

SW-Plot verschiedener GaN:Gd Schichten. Volle Symbole stehen für zur Zeit der Messung ferromagnetische, offene für nicht ferromagnetische Proben. Kreuze stehen für die Probe J0188 auf $\mathrm{SiC}-$ Template. Auffällig ist die für die Schicht J0175 niedrige $\mathrm{V}_{\mathrm{Ga}}$ Konzentration. Der Punkt der J0175 liegt dabei schon jenseits des Punktes für Galliumleerstellen-freies GaN. Diese Werte können nicht nur durch eine geringe $\mathrm{V}_{\mathrm{Ga}}$-Konzentration erklärt werden. Die Referenzpunkte sind von der Aalto Universität zur Verfügung gestellte Referenzmessungen. 
und Dev et al. vorgeschlagene Stabilisierung der ferromagnetischen Phase durch Galliumleerstellen ausgeschlossen werden. Diese Beobachtung ist mit den zuvor gezeigten EPRund XANES-Messungen im Einklang, welche keine Veränderungen der GaN-Matrix in der Umgebung der Gd-Atome feststellen konnten.

Ein Hinweis auf die Art des Defektes, welcher für die magnetische Ordnung in GaN:Gd eine Rolle spielen könnte ist in den Tiefenprofilen des S-Parameters zu finden. In Abbildung 5.5(b) ist dieser gegen die Implantationsenergie der Positronen aufgetragen. Die Implantationsenergie ist ein Maß für die Einpflanztiefe der Positronen und so erhält man ein Tiefenprofil des S-Parameters. Eine Positronenenergie von ca. $15 \mathrm{keV}$ entspricht einer Einpflanztiefe von ca. $500 \mathrm{~nm}$, was wiederum der Dicke der Schichten entspricht. Für niedrige Energien wird ein steiler Abfall des S-Parameters gefunden. Dieser ist dem schnell in die Tiefe abklingenden Einfluß des Oberflächenzustandes der Positronen zuzuordnen. Das darauffolgende Plateau ist der S-Parameter der GaN:Gd-Schicht und entspricht dem in Abbildung 5.5(a) aufgetragenen Wert des S-Parameters. Für größere Einpflanztiefen geht der S-Parameter auf den Substratwert über. Als Referenz für den GaN-Volumenkristall ist als graue Linie eine $\mathrm{V}_{\mathrm{Ga}}$-freie GaN:Mg-Schicht gezeigt. Auffällig ist ein deutlich schneller abfallender Oberflächenzustand in der Probe J0175. Dies ist der Fingerabdruck eines Akzeptors [62]. Mit temperaturabhängigen Messungen könnte dieser noch genauer charakterisiert werden. Entsprechende Messungen werden zur Zeit an der Aalto Universität durchgeführt.

\subsection{Optische Eigenschaften}

Die PL ist eine mächtige Methode, um die Defektlandschaft in einem Halbleiter zu charakterisiern. Besonders im Bezug auf GaN ist hier die YL, ein breites Band um ca. 2,2 eV zentriert, zu nennen. Der Ursprung der YL ist nicht abschließend geklärt. Als breiter Konsenz gilt aber die Galliumleerstelle, bzw. die mit Sauerstoff dekorierte Galliumleerstelle. Einen Überblick über die optischen Eigenschaften von GaN liefert [33].

Die PL Messungen dieser Arbeit wurden in einem He-Badkryostaten durchgeführt. Durch Absenkung des Druckes über dem Helium wird der superfluide Zustand erreicht und damit Temperaturen unterhalb von $2 \mathrm{~K}$. Die Anregung findet mit einem $\mathrm{He}-\mathrm{Cd}$ Laser bei $325 \mathrm{~nm}$ statt. Die Leistung des Lasers beträgt $45 \mathrm{~mW}$. Durch die nicht für UVMessungen optimalen Fenster des Kryostaten wird diese jedoch reduziert. Die Spektren wurden mit einem Acton Research Corporation SpectraPro 750 Spektrometer und einem charge coupled device (CCD) aufgenommen und sind Untergrundkorrigiert.

der Probe gegenüber der Referenz schließen. Die Ursache hierfür ist zur Zeit unbekannt und in Untersuchung. 
Alle Messungen der Proben mit Nummern kleiner als J0176 und der Probe G0135 wurden von Jan Zenneck im Rahmen seiner Dissertation durchgeführt [63].

\subsection{1 Übersichtsspektren}

Abbildung 5.6 zeigt eine Übersicht der PL-Spektren von ausgesuchten Proben. Aus Gründen der Übersichtlichkeit sind die Daten auf der y-Achse gegeneinander verschoben. Von unten beginnend sind zwei Referenzproben gezeigt. Sie stammen aus einer VEECO Mod. Gen II MBE (G0135) und aus der Anlage ELSA (J0171). Beide zeigen eine ausgeprägte bandkantennahe Lumineszenz bei ca. 3,5eV und verschiedene Signale zwischen 3,0 und 3,4 eV. Diese könnten einem DAP-Übergang (donor-acceptor-pair) und den sog. $Y$-Linien zugeordnet werden, welche vermutlich mit ausgedehnten strukturellen Defekten zusammenhängen [33]. Außerdem ist bei ca. 2,2 eV die für GaN bekannte YL zu erkennen [33]. Zusätzlich ist für die in der Gen II hergestellte Probe (G0135) ein ausgeprägtes rotes Lumineszenzband (RL) bei ca. 1,8 eV zu erkennen. Auch die Herkunft der RL ist nicht abschließend geklärt. Beobachtet wird sie in einem breiten Feld von GaNSchichten. Ihre Herkunft wird je nach Wachstumsmethode und den verwendeten Wachstumsparametern mit nativen Defekten (ausgedehnt) oder Verunreinigungskomplexen in Verbindung gebracht. Eine Charakteristik ist aber allen beobachteten RL gemein. Ihr Verhalten läßt auf die Anwesenheit eines tiefen Akzeptors in den GaN-Schichten schließen. In diesem Zusammenhang wird die RL auch häufig in stark kompensierten und damit semi-isolierenden oder isolierenden Schichten beobachtet [33].

Weiter aufsteigend sind repräsentative ferromagnetische Schichten beider Serien gezeigt (J0176 - J0264). Der Vergleich mit den Referenzen bringt für diese Proben keine neuen Signale, welche eindeutig mit dem Gd-Einbau verknüpft werden können. Es kann weder die von Wysmołek et al. gefundene $\mathrm{Gd}^{3+}$ Luminiszenz bei ca. $1,8 \mathrm{eV}$ und ca. $3,35 \mathrm{eV}$ [64], noch das von Choi et al. beobachtete Signal bei ca. 1,9 eV (650 nm) [65], bestätigt werden. Es fällt auf, daß alle Proben ausgeprägte YL und teils RL aufweisen, welche aber nicht mit Gd-Konzentration oder der Magnetisierung korrelieren.

In den Proben J0172 und J0175 ist eine starke RL vorhaden. Diese beiden Schichten zeigten schon in der absoluten Sättigungsmagnetisierung hohe Werte. Probe J0172 hat nach ca. 1 Jahr keine Sättigungsmagnetisierung mehr aufgewiesen, Probe J0175 ist von der Alterung ausgenommen und fiel in der PAS mit einem Verhalten auf, welches auf eine besonders niedrige Konzetration von $\mathrm{V}_{\mathrm{Ga}}$ bzw. $\mathrm{V}_{\mathrm{Ga}}-\mathrm{O}_{\mathrm{N}}$ und die Anwesenheit eines Akzeptors in der Schicht schließen läßt. Die oft mit Akzeptoren in Verbindung gebrachte RL [33] ist damit für Probe J0175 in Einklang mit der PAS. Es muß an dieser Stelle erwähnt werden, daß auch die G0135, also die nicht mit Gd dotierte GaN-Referenz eine RL aufweist. Auch kann die RL nicht mit dem Auftreten einer Magnetisierung korreliert werden, wie gut in der Übersicht über die PL-Spektren zu erkennen ist. Weiter muß 
beachtet werden, daß die Messungen von PAS ca. 2 Jahre nach der PL stattfanden und aufgrund der Alterungseffekte eine Veränderung in der PAS oder PL nicht auszuschließen ist.

Die vier letzten Spektren in Abbildung 5.6 sind nicht magnetische Proben der Serie 2. Aufsteigend von unten nach oben nimmt die Gd-Konzentration in der Schicht zu. Gleichzeitig ist auch eine deutliche Zunahme der YL zu verzeichnen. Für die am höchsten mit Gd dotierte Schicht $\left(\mathrm{J} 0261, \mathrm{c}_{\mathrm{Gd}}=2,7 \cdot 10^{18} \mathrm{~cm}^{-3}\right)$ findet sich zusätzlich ein breites Band zentriert um 3,2 eV. Das Auftreten dieses Bandes nur in der hoch dotierten Schicht legt die Vermutung von Gd-haltigen Auscheidungen als Grund nahe. Eine Untersuchung mit XRD oder TEM steht zur Zeit aus.

Vergleicht man ferromagnetische und nicht ferromagnetische Schichten, so fällt allgemein auf, daß in allen ferromagnetischen Schichten deutlich ausgeprägtere Defektlumineszenzen (YL, RL, BL) zu finden sind. In allen nicht ferromagnetischen Schichten dominiert die bandkantennahe Lumineszenz, bei ferromagnetischen ist diese vergleichbar mit der YL oder RL, teils sogar schwächer. Der allgemeine Vergleich der PL-Spektren unterstreicht die wichtige Rolle von Defekten in magnetischem GaN:Gd, kann aber hier keine direkten Hinweise auf die Identität der relevanten Defekte geben.

\subsubsection{Bandkantennahe Lumineszenz}

Abbildung 5.7 zeigt eine Vergrößerung der bandkantennahen Lumineszenz der Spektren aus Abbildung 5.6. Die gezeigten Zuordnungen der unterschiedlichen Signale zu freien und gebundenen Exzitonen entsteht durch die in diesem und dem folgenden $\mathrm{Ab}$ schnitt gemachten Beobachtungen von Trends und Vergleichen. Vorgreifend ist zu sagen, daß keine der Zuordnungen sicher ist, sondern einer Bestätigung durch weitere Messungen, wie z.B. Temperatur- und Anregungsleistungsabhängigkeit, bestätigt oder widerlegt werden müssen.

In der GaN-Referenz G0135 ist die Zuordnung der exzitonischen Übergänge möglich und wurde von Jan Zenneck in seiner Dissertation vorgenommen [63]. Dies sind die beiden freien Exzitonen $\mathrm{FX}_{\mathrm{A}}$ und $\mathrm{FX}_{\mathrm{B}}$ bei $3,488 \mathrm{eV}$ und $3,497 \mathrm{eV}$, sowie ein angeregter Zustand des freien A-Exzitons $\mathrm{FX}_{\mathrm{A}}^{\mathrm{n}=2}$ bei $3,507 \mathrm{eV}$. Darüber hinaus kann das donatorgebundene Exziton $\mathrm{D}^{0} \mathrm{X}_{\mathrm{A}}$ bei $3,481 \mathrm{eV}$ beobachtet werden. In Probe J0171 kann nur das donatorgebundene Exziton $\mathrm{D}^{0} \mathrm{X}_{\mathrm{A}}$ bei $3,483 \mathrm{eV}$ gefunden werden. Diese Werte werden im folgenden als Referenz für in der Anlage ELSA hergestelltes GaN auf MOCVD-GaNTemplates verwendet.

Für nicht ferromagnetische Schichten (J0257 - J0261, steigende Gd-Konzentration) liegt die Energie der bandkantennahen Lumineszenz oberhalb der Energie des donatorgebundenen Exzitons $\mathrm{D}^{0} \mathrm{X}_{\mathrm{A}}$ der Referenzen $(3,482 \mathrm{eV})$. Es liegt hier die Vermutung nahe, daß das beobachtete Signal das $\mathrm{D}^{0} \mathrm{X}_{\mathrm{A}}$ Signal ist und sich dieses mit der Gd- 


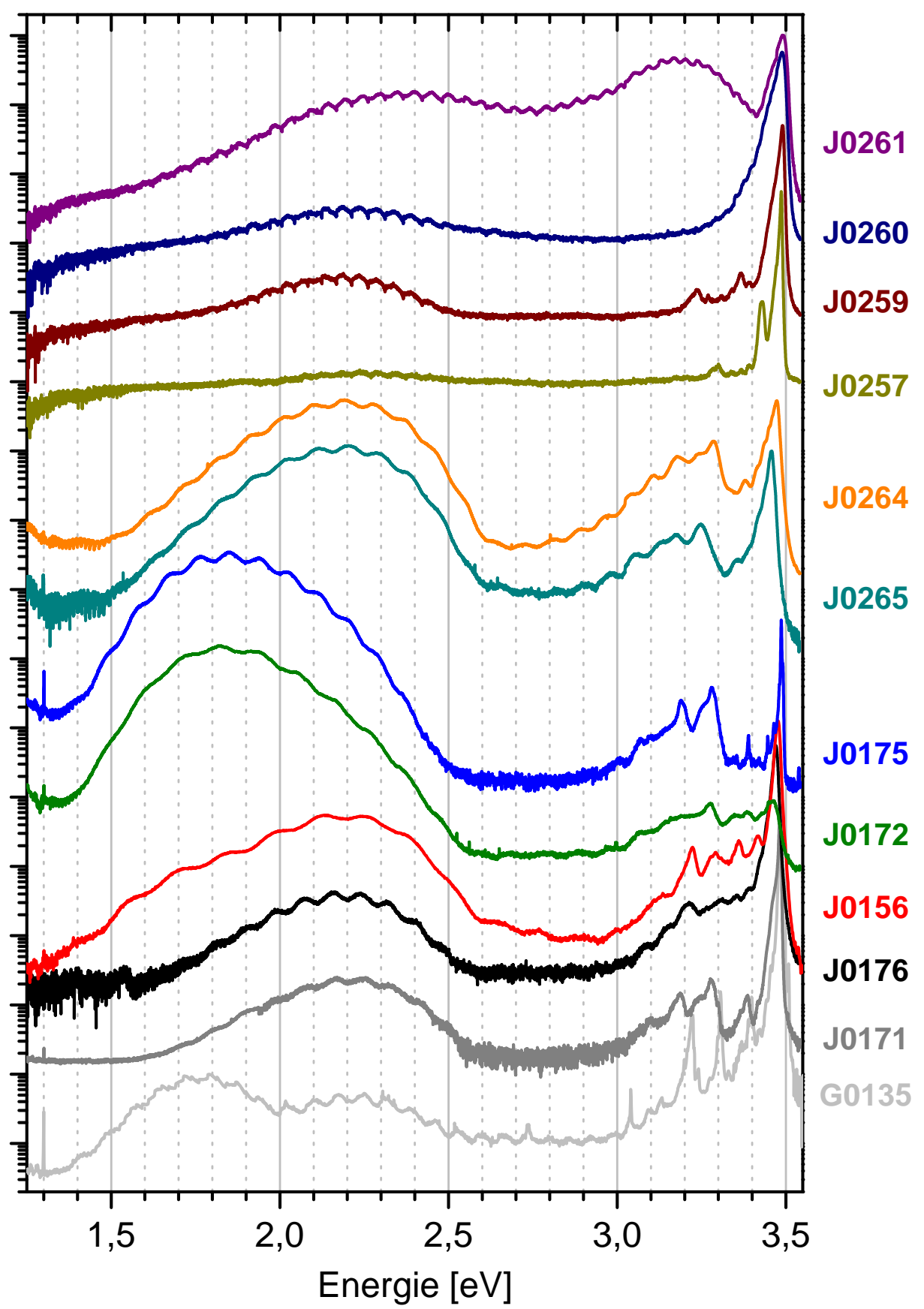

Abbildung 5.6:

Übersicht über die PL-Spektren. Die Proben G0135 und J0171 sind GaN-Referenzen, J0176 J0264 sind ferromagnetische Proben, J0257 - J0261 sind nicht ferromagnetisch. 


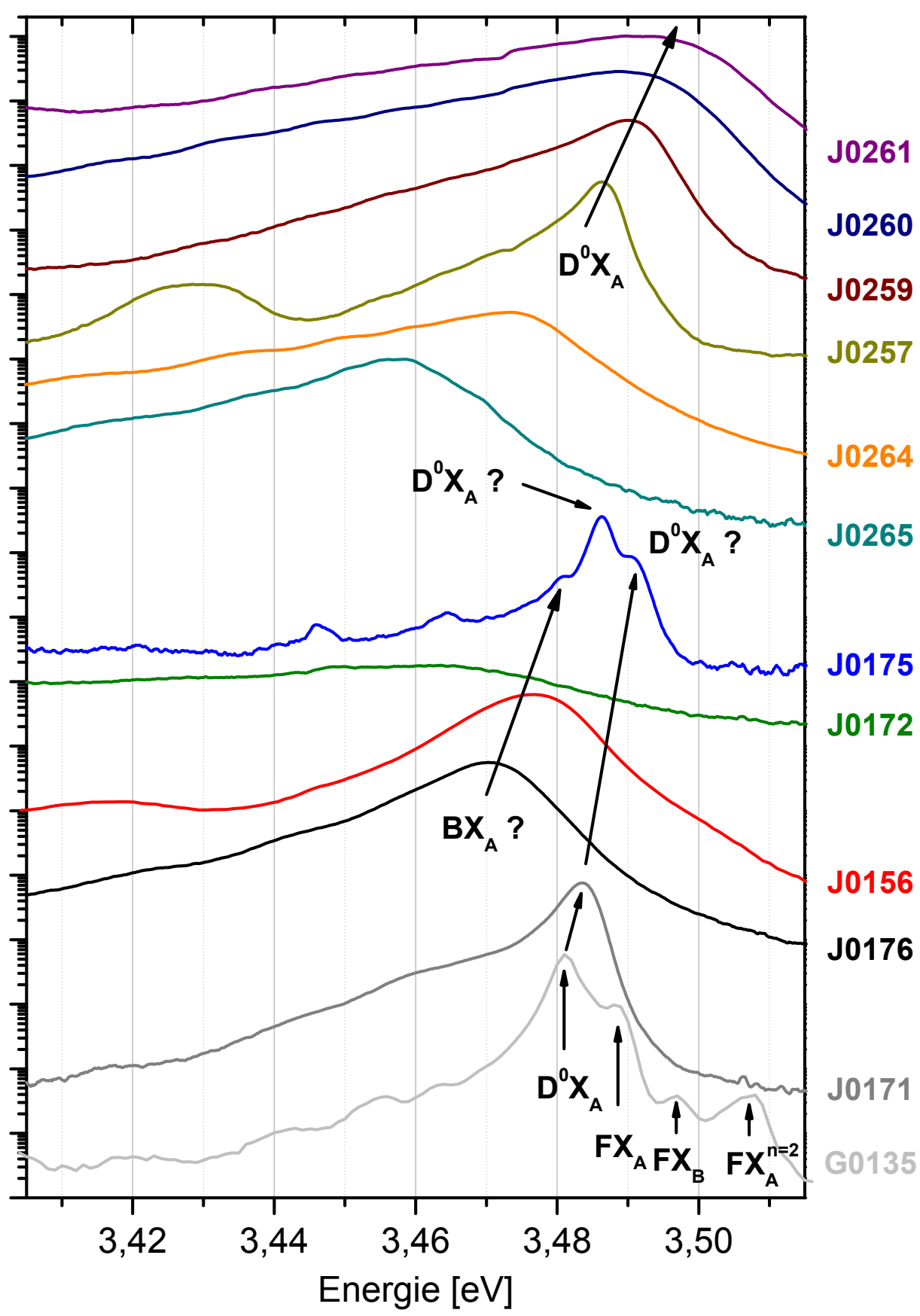

Abbildung 5.7:

Übersicht über die bandkantennahe Photolumineszens. Die Proben G0135 und J0171 sind GaNReferenzen, J0176 - J0264 sind ferromagnetische Proben, J0257 - J0261 sind nicht ferromagnetisch. Die gezeigten Zuordnungen sind nur für die GaN-Referenzen als sicher anzusehen. Die gezeigten Trends und Zuordnugnen der Gd-dotierten Schichten sind im Text diskutiert. 
Konzentration zu höheren Energien verschiebt. Als der Grund für die Verschiebung kann eine biaxiale Verspannung der Schicht mit dem Einbau des deutlich größeren Gd-Atoms in die GaN-Matrix angenommen werden, welche wie folgt zu stande kommt. In unverspanntem GaN (ohne unterliegendes Template) sind $a_{0}$ und $c_{0}$ die Gitterkonstanten für die ensprechenden Kristallrichtungen. Bringt man (immernoch ohne unterliegendes Template) Gd in die GaN-Matrix ein, so vergrößern sich die Gitterkonstanten zunehmend mit der Gd-Konzentration; $a_{\mathrm{Gd}}>a_{0}$ und $c_{\mathrm{Gd}}>c_{0}$. Wächst dieses Material jetzt auf einem Saphir-Substrat oder GaN-Template auf Saphir, so wird eine biaxiale Verspannung eingeführt. Diese steigt mit der Gd-Konzentration aufgrund der gegenüber dem undotierten GaN größeren Gitterkonstante $a_{\mathrm{Gd}}$ an. Wie von Shikanai et al. beobachtet folgen die freien Exzitonen dieser Dehnung linear [66]. Nimmt man $\mathrm{D}^{0} \mathrm{X}=3,470 \mathrm{eV}^{2}$ als Referenz für unverspanntes GaN an, so ergeben sich nach Shikanai et al. für die Dehnung entlang der $c$-Achse Werte von $\epsilon_{\mathrm{zz}}=7,1 \cdot 10^{-4}$ für die GaN-Referenzproben bis hin zu $\epsilon_{\mathrm{zz}}=13,6 \cdot 10^{-4}$ für die Probe J0261. Entsprechend lassen sich nach Davydov et al. so Werte für die Verspannung $\sigma_{\mathrm{zz}}$ von 0,55 bis $1,1 \mathrm{GPa}$ berechnen [68].

Für ferromagnetisches GaN:Gd beider Serien (J0176 - J0264) sind die exitonischen Lumineszenzen gegenüber der Referenz deutlich zu niedrigeren Energien verschoben. Dabei zeigen leicht dotierte Schichten eine sehr breite Lumineszenz, während die am höchsten dotierte Schicht (J0175) schärfere Signale zeigt. Diese Probe zeigte auch schon bei der magnetischen Alterung und in der PAS abweichende Ergebnisse, da die Magnetisierung auch noch nach ca. 3 Jahren beobachtet werden konnte und es keine Anzeichen für nennenswerte $\mathrm{V}_{\mathrm{Ga}}$ Konzentrationen gab. In Probe J0172 ist keine ausgeprägte bandkantennahe Lumineszenz zu erkennen. Die in der Abbildung zugeordneten exzitonischen Lumineszenzen gehen aus der Auswertung der Signale im Vergleich zur Gd-Konzentration hervor und sind in Abschnitt 5.4.3 diskutiert.

Bis jetzt noch nicht betrachtet wurde die Lumineszenz der Sauerstoff-co-dotierten Schichten (J0273-J0275). Diese sind interessant, da dort eine Korrelation mit den magnetischen Eigenschaften und dem Sauerstoffangebot während des Wachstums festgestellt werden konnte (Abschnitt 4.4.3). Abbildung 5.8 zeigt die bandkantennahe Lumineszenz dieser drei Schichten. Von unten nach oben steigt das Sauerstoffangebot, die beiden oberen Schichten sind ferromagnetisch, die untere zeigt nur eine sehr schwache Magnetisierung (vgl. Abbildung 4.6). Deutlich ist das aus mehreren Beiträgen bestehende Band bei ca. 3,44 bis 3,47 eV in der am höchsten mit Sauerstoff co-dotierten Schicht J0273 zu erkennen. Da keine Daten über das Temperaturverhalten oder die Änderung der Signale mit der Anregungsleistung vorliegen, können nur Vergleiche mit anderen Veröffentlichungen und den vorliegenden Referenzen gemacht werden. Sauerstofflumineszenzen wurden von Freitas et al. und von Korotkov et al. untersucht [67, 69]. TES-

\footnotetext{
${ }^{2} 8 \mathrm{meV}$ unterhalb des $\mathrm{FX}_{\mathrm{A}}$ in unverspanntem GaN, siehe [66] und [67]
} 


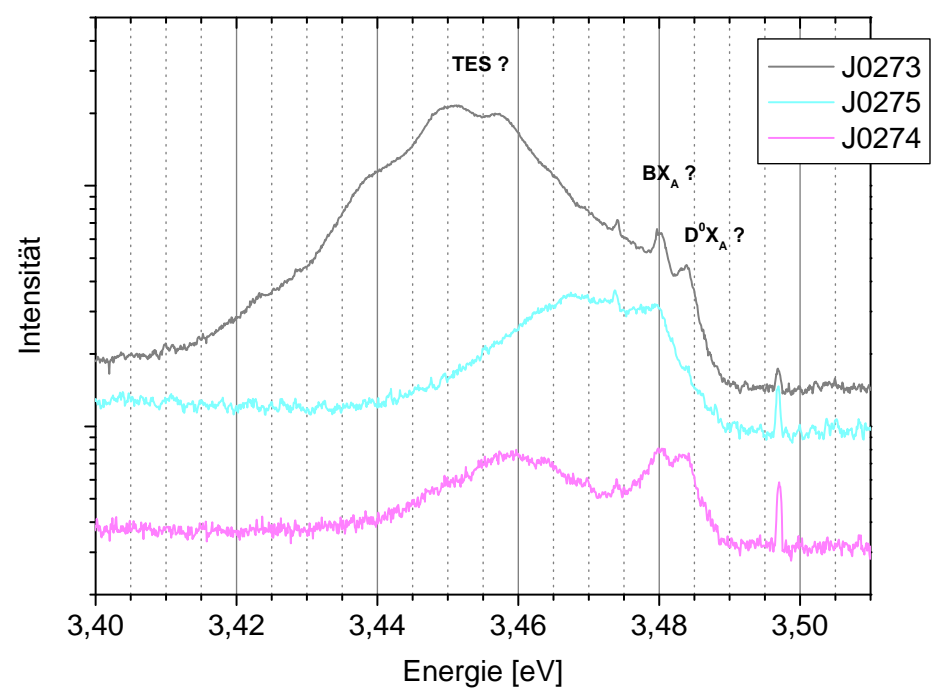

\begin{abstract}
Abbildung 5.8:
Bandkantennahe Lumineszens von GaN:Gd:O. Von unten nach oben steigt das Sauerstoffangebot während des Wachstums. Die beiden obeneren Spektren gehören zu ferromagnetischen Schichten, die Schicht zum unteren Spektrum weist nur ein sehr schwache Magnetisierung auf. Die Zuordnungen der Signale entsprechen denen aus den Trends mit der Gd-Konzentration gewonnenen.
\end{abstract}

Linien (two electron satellites) entstehen, wenn nach der Rekombination eines gebundenen Exzitons nicht der Grundzustand des bindenden Donators/Akzeptors zurückbleibt, sondern ein angeregter Zustand. Dieser ist dann gerade um die Ionisationsenergie zu niedrigeren Energien verschoben. Legt man die von Freitas et al. gefundenen Abstände der verschiednen TES-Linien des Sauerstoffdonators $\mathrm{O}_{\mathrm{N}}$ zugrunde, so können die beiden stärksten Signale des breiten Bandes bei 3,450 eV und 3,457 eV den beiden stärksten TES-Linien des Sauerstoffdonators zugeordnet werden, das donatorgebundene Exziton $\mathrm{D}^{0} \mathrm{X}_{\mathrm{A}}$ ist dann das Signal bei $3,480 \mathrm{eV}$ und das freie Exziton ist nicht sichtbar. Ein Problem bei dieser Zuordnung sind die Intensitäten der gefundenen Signale. Da die Rekombination der TES-Exzitonen einen weiteren Prozeß, die Ionisation der Störstelle, beinhaltet, ist sie unwahrscheinlicher als die Rekombination des gebundenen Exzitons. Damit sollten ihre Intensität immer unterhalb der gebundenen Exzitonen liegen. Dies ist hier nicht der Fall und machen die TES-Linien als Ursrpung des breiten Bandes unwahrscheinlich. Die in der Abbildung 5.8 gezeigten Zuordnungen gehen, wie die der ferromagnetischen Schichten aus Abbildung 5.7, aus der im nächsten Abschnitt folgenden Diskussion der Gd-Konzentrationsabhängigkeit hervor. Der Ursprung des breiten Bandes bleibt weiter unklar. Hyphotesen könnten die Bindung eines Exzitons an einen Komplexes aus Gd und Sauerstoff beinhalten. 


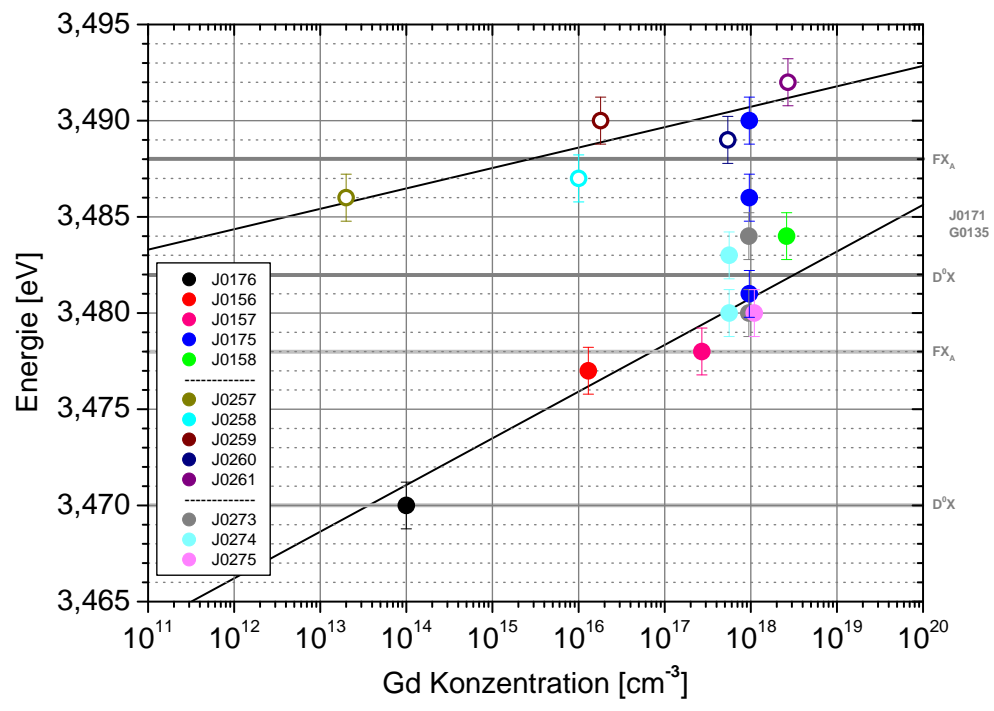

\begin{abstract}
Abbildung 5.9:
Abhängigkeit der bandkantennahen Lumineszens von der Gd Konzentration. Geschlossene Symbole stehen für ferromagnetische Schichten, offene für nicht ferromagnetische Schichten. Die eingezeichneten Geraden sind als Orientierungshilfe zu verstehen. Am Rand sind in dunkelgrau die Referenzen dieser Arbeit und in hellgrau unverspanntes GaN aus [66] gezeigt.
\end{abstract}

\title{
5.4.3 Trends der exzitonischen Lumineszenzen
}

Um die exzitonischen Lumineszenzen mit den magnetischen Eigenschaften und der GdDotierung zu vergleichen, wurden in Abbildung 5.9 die Positionen der jeweils drei bandkantennahen Lumineszenzen (falls vorhanden) gegen die Gd-Konzentration in der Schicht aufgetragen. Die Farben der Punkte korrespondieren mit denen der Spektren aus den vorherigen Abbildungen. Geschlossene Symbole stehen für ferromagnetische Proben, offene für nicht ferromagnetische. Die dunkelgrauen Linien stehen für die vorgestellten GaN Referenzen, in hellgrau ist vollständig relaxiertes GaN nach [66] und [50] eingetragen. Die beiden Geraden sind als Hilfe anzusehen und nicht als physikalische Repräsentation der Daten.

Für alle Schichten ist jeweils die Verschiebung der Exzitonenenergie zu höheren Energien mit steigender Gd-Konzentration zu erkennen. Dabei liegen alle nicht ferromagnetischen Schichten bei deutlich höheren Energien als ferromagnetische. Eine erste Vermutung, daß es sich jeweils um den gleichen $\mathrm{D}^{0} \mathrm{X}_{\mathrm{A}}$ Übergang handelt, welcher durch einen unbekannten Effekt in den ferromagnetischen Schichten weiter beeinflußt wird, scheint hier bei der Betrachtung der Probe J0175 nicht sinnvoll. Diese hat Signale, welche auf beiden der angedeuteten Trendgeraden liegen. Daher wird vorgeschlagen, daß es sich bei der unteren Trendgeraden um ein anderes mit der beschriebenen Verspannung ver- 
schiebendes gebundenes Exziton $\mathrm{BX}_{\mathrm{A}}$ handelt. Ein Hinweis für diese Vermutung ist in der Position des Signals der Probe J0176 zu finden. Diese liegt deutlich unterhalb der Energie des $\mathrm{D}^{0} \mathrm{X}_{\mathrm{A}}$ Übergangs in den Referenzschichten und wäre nur mit Verspannungseffekten nur durch einen entsprechenden $\mathrm{D}^{0} \mathrm{X}_{\mathrm{A}}$ Übergang in vollständig relaxiertem GaN zu erklären [66]. Da aber in nicht ferromagnetischen Schichten für Gd-Konzentrationen von $10^{14} \mathrm{~cm}^{-3}$ schon Einflüsse des Gd in der Verspannung zu erkennen waren und auch eine komplett unverspannte Schicht auf GaN-Templates auf Saphir unwahrscheinlich erscheint $^{3}$, muß für dieses Signal ein in der Energie tiefer liegendes Signal oder ein weiterer unbekannter Effekt der Ursprung sein. Spekulativ könnte man anhand der Hinweise auf einen Akzeptor, z.B. in der PAS, den für ferromagnetische Proben beobachteten Übergang einem akzeptorgebundenen Exzition $\mathrm{A}^{0} \mathrm{X}_{\mathrm{A}}$ zuordnen. Dieses müßte mindestens eine Bindungsenergie von $8 \mathrm{meV}$ haben (Unterschied des Signals der Probe J0176 zum $\mathrm{FX}_{\mathrm{A}}$ der Referenz aus [66]). Freitas et al. finden in ihren Schichten ein Exziton, welches an einen unbekannten Akzeptor gebunden ist, ca. $13 \mathrm{meV}$ unterhalb des freien Exzitons und Korotkov et al. ca. $20 \mathrm{meV}$ unterhalb. Diese Bindungsenergien eines Exzitons an einen Akzeptor entsprechen nach Haynes Regel einer Ionisationsenergie des Akzeptors von ca. $200-400 \mathrm{meV}$ [33]. Dieses würde zur Ionisationsenergie des $\mathrm{O}_{\mathrm{i}}$ passen, welche unterhalb von $500 \mathrm{meV}$ liegt (Abbildung 1.4, [40]). Eine sichere Zuordnung dieser bandkantennahen Lumineszenzen ist aber nur mit einer intensiveren Studie der PL möglich. Interessant wären hier Temperatur- und Leistungsabhängige Messungen an einem ausgedehnten Set an Gd-dotiertem GaN und Sauerstoff-co-dotiertem GaN:Gd. Bei der Herstellung dieser Schichten sollte besonders die ungewollte Verunreinigung mit Sauerstoff vermieden werden.

\section{Zusammenfassung}

Anhand von XRD-Messungen und TEM-Aufnahmen konnte gezeigt werden, daß die Kristalqualität nur wenig von der Gd-Dotierung beeinträchtigt wird und es keine Ausscheidungen von Gd-haltigen Phasen gibt. Röntgenfluoreszenz, -absorptions und EPRMessungen zeigen unabhängig voneinander, daß Gd auf einem Ga-Platz eingebaut wird und im Ladungszustand 3+, also isovalent zu Ga, vorliegt. Anhäufungen von $\mathrm{V}_{\mathrm{Ga}}$, wie in Abschnitt 1.6 diskutiert, können als Grund für die kolossalen Momente ausgeschlossen werden. Die Positronenvernichtung an den GaN:Gd-Kristallen zeigt keine Korrelation der $\mathrm{V}_{\mathrm{Ga}}$-Konzentration mit der Magnetisierung oder der Gd-Konzentration. Für nicht ferromagnetische Proben können große Konzentrationen an Defekten mit Leervolumen gefunden werden. Es wurde dabei auf Cluster der Galliumleerstelle geschlossen. In einer

\footnotetext{
${ }^{3}$ Paskov et al. finden für eine $80 \mu \mathrm{m}$ dicke GaN-Schicht auf Saphir Einflüsse einer biaxialen Verspannung
} aufgrund des Templates [50]. 
ferromagnetischen Schicht konnte gezeigt werden, daß die Galliumvakanz für die Ursache der magnetischen Kopplung in GaN:Gd ausgeschlossen werden kann. Tiefenprofile des S-Parameters geben einen Hinweis auf einen Akzeptor in ferromagnetischem GaN:Gd. PL-Messungen liefern keine direkten Übergänge des Gd in GaN. Die in den Arbeiten von Lipinska oder Wysmołek gefundenen $\mathrm{Gd}^{3+}$ Lumineszenzen können nicht bestätigt werden [70, 64]. Vergleiche von ferromagnetischem und nicht ferromagnetischem Material zeigen, daß vor allem die YL und die RL besonders in ferromagnetischem Material sehr ausgeprägt sind und unterstreichen das Bild von Defekt-induziertem Magnetismus in GaN:Gd. Eine Analyse der exzitonischen Zustände nahe der Bandkante zeigt eine Abhängigkeit von der Gd-Konzentration, welche nicht alleine durch die Verspannung des GaN erklärt werden kann. Für die Klärung der in der PL beobachteten Signale sind weitere Messungen notwendig. 


\section{Zusammenfassung und Ausblick}

Die vorliegende Arbeit untersucht das magnetische Verhalten von GaN:Gd bei $300 \mathrm{~K}$ unter verschiedenen Einflüssen und versucht dieses in Relation mit den in der Literatur gefundenen Ergebnissen zu setzen. Nach einem kurzen Überblick über die in der Literatur gefundenen Eigenschaften von GaN:Gd, wird das Phänomen der kolossalen magnetischen Momente diskutiert. Es wird ein phänomenologisches Modell vorgeschlagen, welches darauf beruht, daß ein Großteil der magnetischen Momente von Defekten in der GaN-Matrix getragen wird, deren Konzentration aber weitestgehend unabhängig von der Gd-Konzentration ist. Die Wechselwirkung wird dabei von Gd induziert und ist nicht durch Gd direkt getragen. Die kolossalen Momente entstehen durch die Umrechnung der von den Defekten getragenen Momente auf das Gd-Atom. Es kann eine Defektkonzentration von ca. $10^{19} \mathrm{~cm}^{-3}$ abgeschätzt werden, welche die Magnetisierung von GaN:Gd trägt. Bei einer Betrachtung der typischen Defekte in GaN und der theoretischen Arbeiten zu GaN fallen dabei besonders die Galliumleerstelle und Sauerstoffverunreinigungen ins Auge. Von verschiedenen Autoren theoretischer Arbeiten wird die Galliumvakanz, bzw. der Komplex aus einer Galliumvakanz und einem Gd-Atom als Ursache für die magnetischen Eigenschaften von GaN:Gd vorgeschlagen [46, 47, 35]. Eine weitere Arbeit schlägt einen Komplex aus Gd und einem Sauerstoffatom vor [49]. Diese Theorie wird von den hohen Sauerstoffkonzentrationen in den in der Literatur untersuchten GaN:Gd-Schichten unterstützt.

Im folgenden wird der Fokus auf die Bestimmung der Gd-Konzentration mit SIMS gelegt. Insbesondere eine Korrektur der Daten auf Masseninterferenzen zeigt ein großes Potential für Fehler in der Gd-Konzentration von ca. einer Größenordnung. Für niedrige Gd-Konzentrationen wurde eine theoretische Abschätzung mit Hilfe der Effusionstheorie gemacht. Es wird gezeigt, daß die hergestellten GaN:Gd-Schichten Gd-Konzentrationen im Bereich von $10^{12}$ bis $10^{20} \mathrm{~cm}^{-3}$ haben. Eine Abschätzung der Verunreinigungen durch Übergangsmetalle aus dem Gd-Quellenmaterial anhand einer hoch Gd-dotierten Schicht ergibt, daß alle Übergangsmetalle mit einer mindestens um den Faktor 1000 kleineren Konzentration vorliegen als das Gd. Messungen der Sauerstoffkonzentration in den Schichten stehen aus.

Für alle nicht co-dotierten ferromagnetischen GaN:Gd-Schichten wird eine zunächst steigende Sättigungsmagnetisierung mit der Gd-Konzentration gefunden. Dabei ist auffällig, daß jeweils für die Schichten mit hohen Gd-Konzentrationen um die $10^{19} \mathrm{~cm}^{-3}$ 
die Sättigungsmagnetisierung verschwindet. Maximale Sättigungsmagnetisierungen werden im Bereich von $10^{17}-10^{18} \mathrm{~cm}^{-3}$ gefunden. Diese Beobachtung wird auch von Hite et al. gemacht [31]. Die kolossalen Momente pro Gd-Atom wie von Dhar et al. gefunden, konnten bestätigt werden. Si-co-Dotierung zeigt keine schlüssigen Einflüsse auf die magnetischen Eigenschaften, allerdings stehen hier auch nur wenige Proben zur Verfügung. H-co-Dotierung reduziert die Sättigungsmagnetisierung, für eine co-Dotierung mit Sauerstoff kann eine steigende Sättigungsmagnetisierung mit steigendem Sauerstoffangebot gefunden werden. Die Reproduzierbarkeit der magnetischen Eigenschaften kann als schlecht bezeichnet werden. Der ausschlaggebende Parameter konnte nicht identifiziert werden, allerdings lassen die Ergebnisse der Sauerstoff-co-Dotierung die Vermutung von Sauerstoffverunreinigungen während des Wachstumsprozesses zu. Anhand einer Probe mit GdN-Ausscheidungen konnte gezeigt werden, daß diese nicht für die magnetischen Eigenschaften in GaN:Gd verantwortlich sein können und so die Möglichkeit eines geblockten Superparamagneten ausgeschlossen werden. Die Alterung der magnetischen Eigenschaften wurde über einen Zeitraum von 4 Jahren untersucht. Verschiedene ferromagnetische Proben verloren innerhalb ca. 1 Jahres vollständig ihre Magnetisierung. Eine Probe ist von dieser Alterung ausgenommen und zeigt nur eine leichte Reduktion der Sättigung. Der Grund ist unbekannt.

Anhand von XRD- und TEM-Messungen konnten weitere Beweise für den Ausschluß von Gd-haltigen Ausscheidungen als Grund für die magnetischen Eigenschaften gefunden werden. Der Ladungszustand des Gd in der GaN-Matrix kann mit EPR zu 3+ bestimmt werden und weiter gezeigt werden, daß in der Umgebung des Gd-Atom keine strukturellen Veränderungen an der GaN-Matrix zu finden sind. Damit kann insbesondere die Theorie einer Anhäufung von Galliumleerstellen um das Gd-Atom als Grund für die kolossalen Momente widerlegt werden [46, 35]. Weiter kann mit Positronenvernichtung gezeigt werden, daß gerade in ferromagnetischen GaN:Gd-Schichten eine verschwindende Konzentration an Galliumleerstellen zu finden ist. Damit ist nicht nur eine Anhäufung von Galliumleerstellen widerlegt, sondern auch die Theorie von durch Galliumvakanzen getragenem Magnetismus ausgeschlossen. Darauf werden optische Eigenschaften der GaN:Gd-Schichten gezeigt. In den PL-Spektren kann gezeigt werden, daß in ferromagnetischen Schichten deutlich ausgeprägtere Defektlumineszenzen, wie die YL um 2,2 eV oder die RL um 1,8 eV vorhanden sind als in nicht ferromagnetischen Schichten. Dabei sind aber keine direkten Korrelationen mit den magnetischen Daten oder der Gd-Konzentration möglich. Die bandkantennahe Lumineszenz weist für ferromagnetische und für nicht ferromagnetische Schichten unterschiedliche Trends mit der Gd-Konzentration auf. Eine Interpretation, insbesondere der ferromagnetischen Schichten ist mit den vorliegenden Daten schwierig. Spekulativ wird ein akzeptorgebundenes Exziton diskutiert. 
An dieser Stelle kann die Frage vom Begin dieser Arbeit wieder aufgenommen werden „GaN:Gd - Ein verdünnter magnetischer Halbleiter?“. Die vermutete Antwort auf diese Frage konnte mit Hilfe der gezeigten Ergebnisse bekräftigt werden. Gd-dotiertes GaN ist kein klassischer verdünnter Halbleiter wie in der Einleitung vorgestellt. Die Gd-Dotierung alleine kann die gefundenen magentischen Eigenschaften nicht erklären und es wurden deutliche Hinweise auf Defekt-induzierten Magnetismus in GaN:Gd gefunden. Die von der Theorie vorgeschlagene Galliumvakanz konnte dabei ausgeschlossen werden und die von Mitra und Lambrecht gezeigte Variante eines Gd-O-Komplexes mit schwacher Bindung scheint insbesondere mit Blick auf die Ergebnisse der Sauerstoffco-dotierten Schichten und der gefundenen Alterung der magentischen Eigenschaften durchaus möglich. Auch sprechen hohe Sauerstoffkonzentrationen in den Arbeiten von Dhar etal. und Hite etal. für diese Theorie. Mit der Beantwortung der Frage nach einem verdünnten magnetischen Halbleiter sind auch neue Fragen aufgetaucht. Wie sind die optischen Eigenschaften zu erklären? Welcher Defekt ist für die magnetischen Eigenschaften verantwortlich? Sind die Hinweise auf einen Akzeptor in der PAS weiter mit dem Magnetismus zu korrelieren? Mit Blick auf die Ergebnisse dieser Arbeit scheint der aussichtsreichste Weg eine kontrollierte und systematische Untersuchung der Sauerstoffabhängigkeit der magentischen Eigenschaften von GaN:Gd zu sein. SIMSMessungen der Sauerstoffkonzentration der Proben dieser Arbeit stehen derzeit aus. Sollte sich diese Vermutung erhärten, so bestehen begründete Hoffnungen in GaN:Gd den für die Halbleiter-Spintronik gesuchten magnetischen Halbleiter mit einer Curietemperatur über $300 \mathrm{~K}$ zu finden. 


\section{Anhang $A$}

\section{Abbildungen und Tabellen}

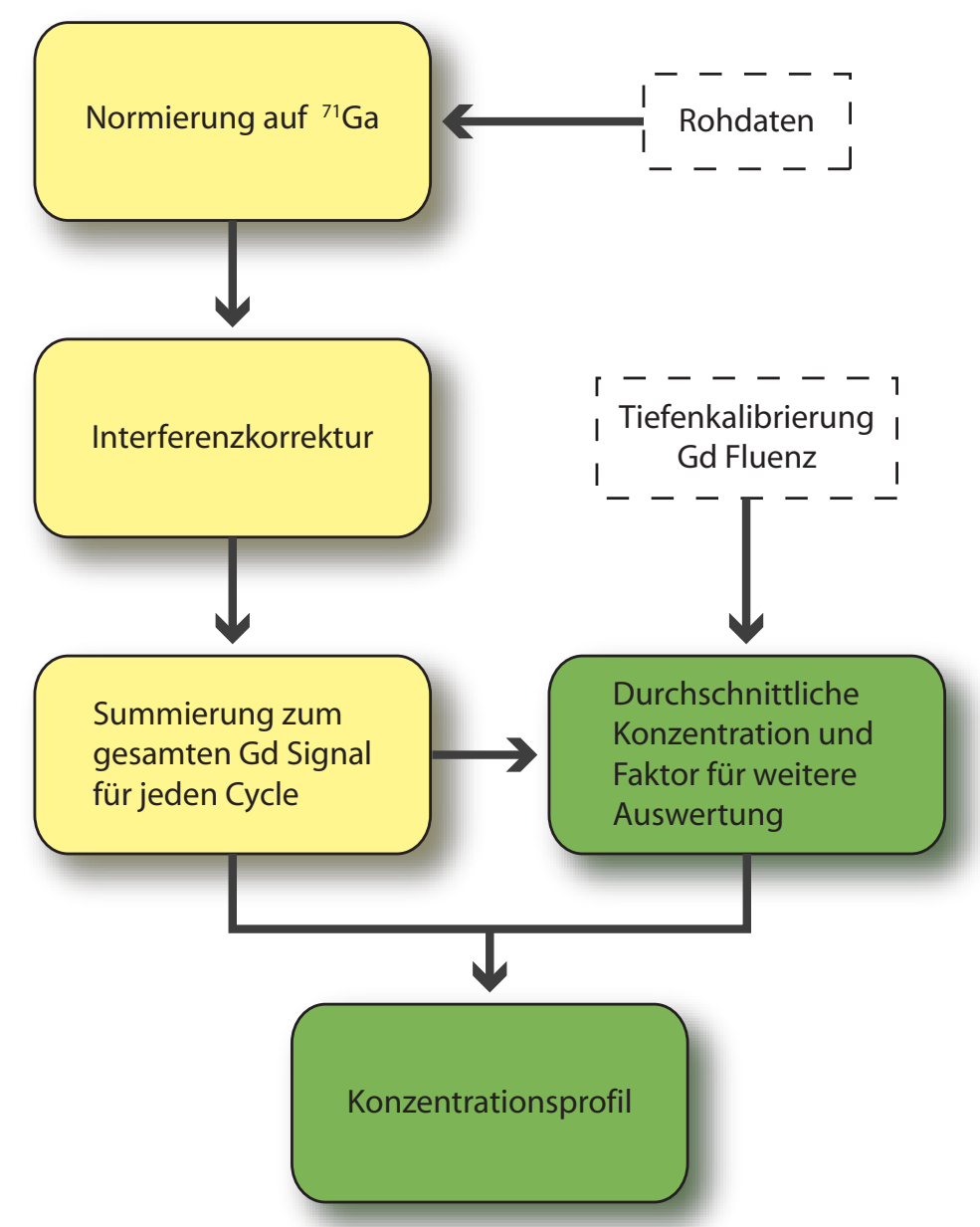

Abbildung A.1: Auswertungsprozedur Standard 


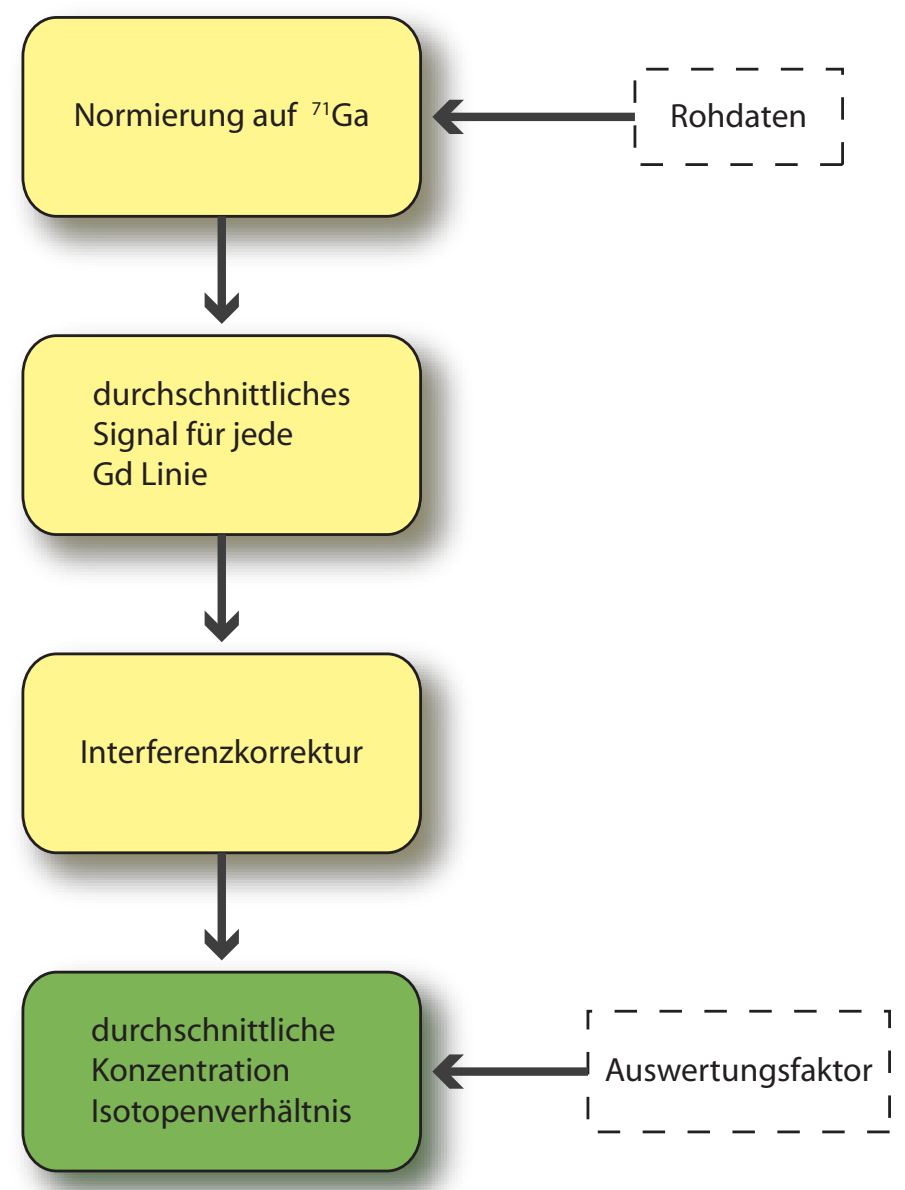

Abbildung A.2: Auswertungsprozedur Proben 
Tabelle A.1: Übersicht über die Proben der Serie 1.

\begin{tabular}{|c|c|c|c|c|c|c|}
\hline \multicolumn{7}{|c|}{$\begin{array}{r}\text { Serie } 1 \\
\mathrm{~T}_{\mathrm{S}}=760^{\circ} \mathrm{C}, \mathrm{BEP}_{\mathrm{Ga}}=5,5 \cdot 10^{-8} \mathrm{mbar} \text {, }\end{array}$} \\
\hline Probe & co-Dot. & $\begin{array}{c}\text { Magnetismus } \\
\text { bei } 300 \mathrm{~K}\end{array}$ & $\begin{array}{l}\text { Sättigung } \\
\text { [emu] }\end{array}$ & $\begin{array}{l}\mathrm{T}_{\mathrm{Gd}} \\
{\left[{ }^{\circ} \mathrm{C}\right]}\end{array}$ & {$\left[\mathrm{cm}^{-3}\right]$} & $\begin{array}{l}\text { Repro } \\
\text { von }\end{array}$ \\
\hline J0156 & & & & 900 & $\left(1,3 \cdot 10^{16}\right)$ & \\
\hline J0157 & & & & 1000 & $2,7(1) \cdot 10^{16}$ & \\
\hline $\mathrm{J} 0158$ & & & & 1100 & $\left(2,6 \cdot 10^{18}\right)$ & \\
\hline $\mathrm{J} 0159$ & & & & 1200 & $1,5(1) \cdot 10^{19}$ & \\
\hline $\mathrm{J} 0172$ & & $\mathrm{fm}$ & $1,1 \cdot 10^{-5}$ & 1000 & $6,0(2) \cdot 10^{16}$ & \\
\hline J0173 & & $\mathrm{nfm}$ & & 1200 & $7,6(2) \cdot 10^{18}$ & \\
\hline $\mathrm{J} 0174$ & & $\mathrm{sp}(<70 \mathrm{~K})$ & & 1400 & $1,6(1) \cdot 10^{20}$ & \\
\hline $\mathrm{J} 0175$ & & $\mathrm{fm}$ & $9,2 \cdot 10^{-6}$ & 1100 & $9,6(3) \cdot 10^{17}$ & \\
\hline $\mathrm{J} 0176$ & & $\mathrm{fm}$ & $7,2 \cdot 10^{-6}$ & 800 & $\left(1,0 \cdot 10^{14}\right)$ & \\
\hline $\mathrm{J} 0177$ & $\mathrm{H}$ & $\mathrm{nfm}$ & & 1100 & $1,9(1) \cdot 10^{17}$ & \\
\hline J0178 & $\mathrm{H}$ & $\mathrm{nfm}$ & & 1400 & $6,3(2) \cdot 10^{19}$ & \\
\hline $\mathrm{J} 0179$ & $\mathrm{H}$ & $\mathrm{fm}$ & $1,7 \cdot 10^{-6}$ & 1000 & $\left(6,1 \cdot 10^{16}\right)$ & \\
\hline J0180 & $\mathrm{H}$ & $\mathrm{fm}$ & $2,7 \cdot 10^{-6}$ & 800 & $\left(1,0 \cdot 10^{14}\right)$ & \\
\hline J0181 & $\mathrm{H}$ & - & & 1200 & $1,5(1) \cdot 10^{18}$ & \\
\hline J0187 & $\mathrm{Si}$ & $\mathrm{fm}$ & $5,5 \cdot 10^{-6}$ & 800 & $\left(1,0 \cdot 10^{14}\right)$ & \\
\hline J0190 & $\mathrm{Si}$ & $\mathrm{nfm}$ & & 1100 & $<2,0 \cdot 10^{16}$ & \\
\hline J0193 & $\mathrm{Si}$ & $(\mathrm{fm})$ & $6,0 \cdot 10^{-7}$ & 675 & $\left(1,0 \cdot 10^{12}\right)$ & \\
\hline J0194 & & $\mathrm{nfm}$ & & 675 & $\left(1,0 \cdot 10^{12}\right)$ & \\
\hline $\mathrm{J} 0210$ & & - & & 1300 & $\left(3,8 \cdot 10^{19}\right)$ & \\
\hline $\mathrm{J} 0211$ & & $(\mathrm{fm})$ & $4,0 \cdot 10^{-7}$ & 1200 & $\left(5,7 \cdot 10^{18}\right)$ & J0173 \\
\hline $\mathrm{J} 0212$ & & $\mathrm{nfm}$ & & 1200 & $\left(5,7 \cdot 10^{18}\right)$ & J0173 \\
\hline $\mathrm{J} 0213$ & & $\mathrm{nfm}$ & & 1200 & $5,2(2) \cdot 10^{18}$ & J0173 \\
\hline $\mathrm{J} 0218$ & & $\mathrm{nfm}$ & & 1200 & $\left(5,7 \cdot 10^{18}\right)$ & J0173 \\
\hline $\mathrm{J} 0226$ & & $\mathrm{nfm}$ & & 1000 & $\left(6,1 \cdot 10^{16}\right)$ & J0172 \\
\hline $\mathrm{J} 0227$ & & $\mathrm{nfm}$ & & 800 & $\left(1,0 \cdot 10^{14}\right)$ & J0176 \\
\hline $\mathrm{J} 0266$ & & $\mathrm{nfm}$ & & 800 & $\left(1,0 \cdot 10^{14}\right)$ & J0176 \\
\hline $\mathrm{J} 0267$ & & $\mathrm{nfm}$ & & 1000 & $\left(6,1 \cdot 10^{16}\right)$ & J0172 \\
\hline $\mathrm{J} 0268$ & & - & & 1100 & $\left(7,5 \cdot 10^{17}\right)$ & J0175 \\
\hline $\mathrm{J} 0269$ & $(\mathrm{H})$ & - & & 1100 & $\left(7,5 \cdot 10^{17}\right)$ & \\
\hline $\mathrm{J} 0270$ & $\mathrm{H}$ & $\mathrm{fm}$ & $1,0 \cdot 10^{-6}$ & 1100 & $\left(2 \cdot 10^{17}\right)$ & \\
\hline $\mathrm{J} 0273$ & $\mathrm{O}$ & $\mathrm{fm}$ & $1,6 \cdot 10^{-6}$ & 1100 & $\left(7,5 \cdot 10^{17}\right)$ & \\
\hline $\mathrm{J} 0274$ & $\mathrm{O}$ & $\mathrm{fm}$ & $6,5 \cdot 10^{-7}$ & 1100 & $\left(7,5 \cdot 10^{17}\right)$ & \\
\hline $\mathrm{J} 0275$ & $\mathrm{O}$ & $\mathrm{fm}$ & $1,5 \cdot 10^{-6}$ & 1100 & $\left(7,5 \cdot 10^{17}\right)$ & \\
\hline
\end{tabular}

Im Kopf sind die standardmäßig verwendeten Wachstumsparameter aufgelistet. Das magnetische Verhalten ist in Spalte „Magnetismus bei $300 \mathrm{~K}$ " zusammenge$\mathrm{faßt}$, dabei steht $\mathrm{fm}$ für eine ferromagnetische Signatur und $\mathrm{nfm}$ für eine nichtferromagnetische Signatur (- keine Daten, () Artefakt). In Spalte "Sättigung“ ist die absolute Sättigungsmagnetisierung bei $300 \mathrm{~K}$ in emu aufgelistet. Gd Konzentrationen in Klammern wurden nach Abschnitt 3.7 abgeschätzt. 
Tabelle A.2: Übersicht über die Proben der Serie 2.

\begin{tabular}{|c|c|c|c|c|c|}
\hline \multicolumn{6}{|c|}{$\begin{array}{r}\text { Serie } 2 \\
10^{-8} \mathrm{mbar},\end{array}$} \\
\hline Probe & Wachstum & $\begin{array}{c}\text { Magnetismus } \\
\text { bei } 300 \mathrm{~K}\end{array}$ & $\begin{array}{c}\text { Sättigung } \\
\text { [emu] }\end{array}$ & $\begin{array}{l}\mathrm{T}_{\mathrm{Gd}} \\
{\left[{ }^{\circ} \mathrm{C}\right]}\end{array}$ & $\begin{array}{c}\mathrm{C}_{\mathrm{Gd}} \\
{\left[\mathrm{cm}^{-3}\right]}\end{array}$ \\
\hline $\mathrm{J} 0236$ & & $\mathrm{nfm}$ & & 1100 & $\left(1,5 \cdot 10^{17}\right)$ \\
\hline $\mathrm{J} 0237$ & $\mathrm{BEP}_{\mathrm{Ga}}=0,75 \cdot 10^{-7} \mathrm{mbar}$ & $\mathrm{nfm}$ & & 1100 & $\left(1,5 \cdot 10^{17}\right)$ \\
\hline $\mathrm{J} 0238$ & $\mathrm{BEP}_{\mathrm{Ga}}=1 \cdot 10^{-7} \mathrm{mbar}$ & $\mathrm{fm}$ & $8,5 \cdot 10^{-7}$ & 1100 & $\left(1,5 \cdot 10^{17}\right)$ \\
\hline J0239 & $\mathrm{BEP}_{\mathrm{Ga}}=1,5 \cdot 10^{-7} \mathrm{mbar}$ & - & & 1100 & $\left(1,5 \cdot 10^{17}\right)$ \\
\hline $\mathrm{J} 0240$ & $\mathrm{BEP}_{\mathrm{Ga}}=0,25 \cdot 10^{-7} \mathrm{mbar}$ & $\mathrm{nfm}$ & & 1100 & $\left(1,5 \cdot 10^{17}\right)$ \\
\hline J0241 & & $(\mathrm{fm})$ & $2,0 \cdot 10^{-7}$ & 1100 & $\left(1,5 \cdot 10^{17}\right)$ \\
\hline $\mathrm{J} 0242$ & $\mathrm{~F}_{\mathrm{N}}=2 \mathrm{sccm}$ & $\mathrm{nfm}$ & & 1100 & $\left(1,5 \cdot 10^{17}\right)$ \\
\hline $\mathrm{J} 0243$ & $\mathrm{~F}_{\mathrm{N}}=2,5 \mathrm{sccm}$ & $\mathrm{nfm}$ & & 1100 & $\left(1,5 \cdot 10^{17}\right)$ \\
\hline $\mathrm{J} 0244$ & $\mathrm{~T}_{\mathrm{S}}=730^{\circ} \mathrm{C}$ & $\mathrm{fm}$ & $7,5 \cdot 10^{-7}$ & 1100 & $\left(1,5 \cdot 10^{17}\right)$ \\
\hline $\mathrm{J} 0245$ & $\mathrm{~T}_{\mathrm{S}}=780^{\circ} \mathrm{C}$ & $(\mathrm{fm})$ & $1,0 \cdot 10^{-7}$ & 1100 & $\left(1,5 \cdot 10^{17}\right)$ \\
\hline $\mathrm{J} 0246$ & & $\mathrm{nfm}$ & & 800 & $\left(2,0 \cdot 10^{13}\right)$ \\
\hline $\mathrm{J} 0247$ & & $\mathrm{nfm}$ & & 1100 & $\left(1,5 \cdot 10^{17}\right)$ \\
\hline $\mathrm{J} 0248$ & $\mathrm{~F}_{\mathrm{N}}=2,5 \mathrm{sccm}, \mathrm{P}_{\mathrm{N}}=275 \mathrm{~W}$ & $\mathrm{nfm}$ & & 1100 & $\left(1,5 \cdot 10^{17}\right)$ \\
\hline $\mathrm{J} 0249$ & $\mathrm{~F}_{\mathrm{N}}=2,5 \mathrm{sccm}, \mathrm{P}_{\mathrm{N}}=275 \mathrm{~W}$ & $\mathrm{nfm}$ & & 800 & $\left(2,0 \cdot 10^{13}\right)$ \\
\hline $\mathrm{J} 0252$ & & $\mathrm{nfm}$ & & 1200 & $1,1(1) \cdot 10^{18}$ \\
\hline $\mathrm{J} 0256$ & & $\mathrm{nfm}$ & & 1100 & $\left(1,5 \cdot 10^{17}\right)$ \\
\hline $\mathrm{J} 0257$ & & $\mathrm{nfm}$ & & 800 & $\left(2,0 \cdot 10^{13}\right)$ \\
\hline $\mathrm{J} 0258$ & & $\mathrm{nfm}$ & & 1000 & $\left(1,5 \cdot 10^{17}\right)$ \\
\hline $\mathrm{J} 0259$ & & $\mathrm{nfm}$ & & 1100 & $1,8(1) \cdot 10^{16}$ \\
\hline $\mathrm{J} 0260$ & & $\mathrm{nfm}$ & & 1200 & $5,4(2) \cdot 10^{17}$ \\
\hline J0261 & & $\mathrm{nfm}$ & & 1300 & $2,7(1) \cdot 10^{18}$ \\
\hline $\mathrm{J} 0264$ & & $\mathrm{fm}$ & $8,2 \cdot 10^{-6}$ & 1200 & $1,3(1) \cdot 10^{18}$ \\
\hline J0265 & & $\mathrm{fm}$ & $9,0 \cdot 10^{-6}$ & 1000 & $2,9(1) \cdot 10^{16}$ \\
\hline $\mathrm{J} 0276$ & & $\mathrm{nfm}$ & & 800 & $\left(2,0 \cdot 10^{13}\right)$ \\
\hline $\mathrm{J} 0277$ & & $\mathrm{fm}$ & $8,5 \cdot 10^{-7}$ & 1350 & $1,9(1) \cdot 10^{19}$ \\
\hline
\end{tabular}

Im Kopf sind die standardmäßig verwendeten Wachstumsparameter aufgelistet. Abweichungen davon sind in der Spalte „Wachstum“ vermerkt. Das magnetische Verhalten ist in Spalte „Magnetismus bei $300 \mathrm{~K}$ " zusammengefaßt, dabei steht $\mathrm{fm}$ für eine ferromagnetische Signatur und $n f m$ für eine nicht-ferromagnetische Signatur (- keine Daten, () Artefakt). In Spalte "Sättigung" ist die absolute Sättigungsmagnetisierung bei $300 \mathrm{~K}$ in emu aufgelistet. Gd Konzentrationen in Klammern wurden nach Abschnitt 3.7 abgeschätzt. 


\section{Anhang B}

\section{Effusion}

Bei der MBE werden thermische Verdampfer eingesetzt, um die Materialien für das Schichtwachstum zur Verfügung zu stellen. Dabei spielt die Erzeugung von Molekularstrahlen durch Verdampfung aus der flüssigen oder festen Phase, die Effusion, eine wichtige Rolle. Mit der Kenntnis der Molekülflüsse aus den Materialzellen können z.B. Wachstumsbedingungen beschrieben und analysiert werden, Dotierungen abgeschätzt und Stickingkoeffizienten bestimmt werden. 


\section{B.1 Effusion idealer Oberflächen}

Die ersten Ergebnisse zur Verdampfung von Elementen aus der flüssigen Phase ins Vakuum gehen auf Hertz im Jahre 1882 zurück [71]. Dabei wurde Quecksilber ins Vakuum verdampft. Diese Experimente wurden von Knudsen aufgegriffen und die Hertz/Knudsen Gleichung aufgestellt, welche die maximale Rate der verdampfenden Teilchen von der Quecksilberoberfläche ins Vakuum beschreibt.

$$
\frac{d n}{d t}=a_{v} A_{e}\left(p_{e q}-p_{v}\right) \sqrt{\frac{N_{A}}{2 \pi M k_{B} T}}\left[s^{-1}\right]
$$

$a_{v}$ empirischer Korrekturfaktor, $A_{e}$ Oberfläche des verdampfenden Materials, $p_{e q}$ Gleichgewichtsdruck der Gasphase über der Oberfläche, $p_{v}$ Hintergrunddruck des Vakuums, $N_{A}$ Avogadrozahl, $k_{B}$ Boltzmann Konstante, $M$ Molmasse, $T$ Temperatur der Flüssigkeit.

Dieser Prozess der Verdampfung ins Vakuum wird als Effusion bezeichnet. Dabei ist die Effusionsrate stark von der Geometrie der Oberfläche und des umgebenden Aufbaus abhängig. Daher auch die Bezeichnung der Gleichung B.1 als die maximale Effusionsrate. Der empirische Korrekturfaktor $a_{v}$ gibt dabei die Abweichung von der idealen Effusion an.

Bisher wurde die Effusion von einer freien Flüssigkeitsoberfläche betrachtet. Langmuir konnte schon 1913 zeigen, daß Gleichung B.1 auch für die Effusion von Festkörpern ins Vakuum gilt. Die Effusion einer freien Oberfläche ins Vakuum wird daher auch als Langmuir Modus der Verdampfung bezeichnet.

Die Effusion in einer MBE Anlage ist aber meistens ähnlicher dem Knudsen Modus der Effusion. Die ideale Knudsen Zelle ist ein Behälter mit der Temperatur T. Innerhalb des Behälters hat die Gasphase über der Oberfläche des Materials einen Gleichgewichtsdruck $p_{e q}$. Die Effusion findet aus einer kleinen Öffnung in der Zelle ins Vakuum statt, wobei die Öffnung klein genug sein muß um nicht das Gleichgewicht der Gasphase zu beeinflußen und die Wände der Öffnung unendlich dünn sein müssen, damit aus der Zelle austretende Teilchen nicht gestreut werden. Unter diesen Bedingungen ist die Effusionsoberläche $A_{e}$ jetzt die Öffnung der Knudsen Zelle und der Korrekturfaktor $a_{v}$ ist immer gleich Eins. Darüber hinaus wird für UHV Systeme die Annahme $p_{v}=0$ gemacht. Es folgt aus Gleichung B.1 die Knudsen Effusionsgleichung.

$$
\Gamma \equiv \frac{d n}{d t}=A_{e} p_{e q} \sqrt{\frac{N_{A}}{2 \pi M k_{B} T}}\left[s^{-1}\right]
$$

Diese Gleichung beschreibt die ideale Knudsenzelle. Reale Zellen weisen allerdings Abweichungen auf, die auf endliche Dicken der Zellenöffnung, nicht im Gleichgewicht befindliche Dampfdrücke, Streuung von Molekülen an Wänden, bzw. der Zellenöffnung 


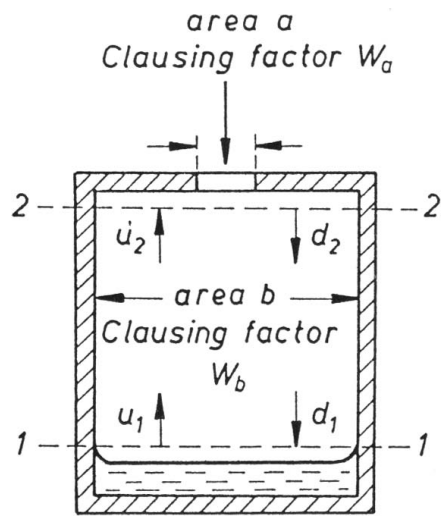

(a) nahezu ideale Knudsenzelle

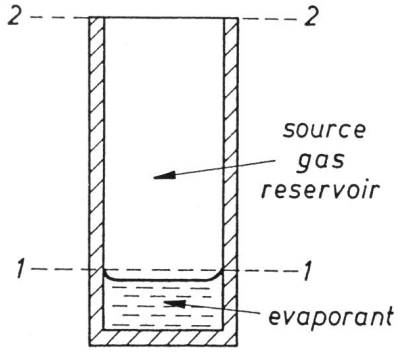

(b) zylindrische Zelle

\section{Abbildung B.1:}

Verschieden Zellengeometrien zu den Korrekturen von Clausing, Motzfeldt und Dayton. Der Durchmesser der area a ist die Größe $d_{0}$, die Dicke des Zellenmaterials an der Stelle der area a ist die Größe $L_{0}$. Abbildungen aus [72].

oder Rekondensation von Material auf den Zellenwänden zurückgeführt werden kann. Für einfache Zellengeometrien können diese Abweichungen wiederum durch Korrekturfaktoren einbezogen werden.

\section{B.2 Korrekturen für reale Effusionszellen}

Effusionszellen in MBE Anlagen entsprechen oft nahezu idealen Knudsenzellen, wie in Abbildung B.1(a) oder Zellen mit offenen Tiegeln wie in Abbildung B.1(b). Ein Beispiel für eine nahezu ideale Knudsenzelle ist die sog. Sumo-Zelle. Die in dieser Arbeit verwendeten Zellen entsprechen dem zweiten Typ, der fortan als zylindrische Zelle bezeichnet werden soll. Für beide Arten von Zellen sind einfache Korrekturen anwendbar, welche Geometrie, Streuung von Teilchen an Wänden und Rekondensation von Molekülen berücksichtigen.

Die erste Korrektur der nahezu idealen Zelle geht auf Clausing zurück, der einen Korrekturfaktor $W_{a}$ einführte. Dieser Clausing Faktor spiegelt die Wahrscheinlichkeit wider, daß ein Teilchen, welches die Zellenöffnung betreten hat, direkt aus der Zelle austritt ohne zwischendurch gestreut zu werden oder nochmals zurück in die Zelle zu wandern. Die Korrektur von Clausing berücksichtigt aber nur die Geometrie der Zellenöffnung und nicht die Geometrie der gesamten Zelle. Basierend auf der Korrektur von Clausing entwickelte Motzfeldt eine allgemeinere Korrektur, die die gesamte Geometrie 
nach Abbildung B.1(a) berücksichtigt.

$$
C^{M o t}=\frac{W_{a}}{1+f\left(\alpha^{-1}+W_{b}^{-1}-2\right)}
$$

$W_{a}$ und $W_{b}$ sind die Clausingfaktoren zu den entsprechenden Flächen $a$ bzw. $b$ (siehe Abbildung B.1(a)). $f$ ist der sog. Fluchtkoeffizient der Zellenöffnung und berechnet sich $\mathrm{zu}$

$$
f=W_{a} \frac{a}{b}
$$

Der Koeffizient $\alpha$ wurde von mehreren Autoren analysiert und beschreibt die Rekondensation von Molekülen auf den Wänden der Zelle und der Zellenöffnung. Hier soll die allgemeine Variante von Dayton zur Anwendung kommen, welche gute Werte für Zellen mit $L_{0}>2 d_{0}$ liefert.

$$
\alpha=\frac{u \sqrt{u^{2}+1}-v \sqrt{v^{2}+1}+v^{2}-u^{2}}{\frac{u\left(2 v^{2}+1\right)-v}{\sqrt{v^{2}+1}}-\frac{v\left(2 u^{2}+1\right)-u}{\sqrt{u^{2}+1}}}
$$

mit $u=\frac{L_{0}}{d_{0}}-v$ und $v=\frac{L_{0} \sqrt{7}}{3 L_{0}+d_{0} \sqrt{7}} . L_{0}$ ist die Dicke der Zellenöffnung und $d_{0}$ der Durchmesser der Zellenöffnung.

Auch zylindrische Zellen ohne eine Verengung an der Zellenöffnung wie in Abbildung B.1(b) dargestellt können in guter Näherung mit dieser Korrektur beschrieben werden. Dabei wird der Raum der Zelle als ein schmaler Streifen direkt über dem Zellenmaterial angenommen (Ebene 1 in Abbildung B.1(b)) und der Raum zwischen den Ebenen 1 und 2 als die Zellenöffnung mit Dicke und Durchmesser $L_{0}$ und $d_{0}$. Ein erfreulicher Nebeneffekt ist, daß durch die Variation von $L_{0}$ jetzt verschiedene Füllgrade der Zelle angenommen werden können.

In einem MBE System sind die Zellen auf einer Halbkugel angeordnet in dessen Zentrum die Probe positioniert ist. Aus geometrischen und Wahrscheinlichkeitsüberlegungen (vorgeführt z.B. in [72]) kann der Teilchenfluß $N$ im Abstand $r$ von der Zelle berechnet werden. Durch die Geometrie der Halbkugel ist des weiteren die Probe immer um einen Winkel $\phi$ gegenüber der Zellenachse gekippt. Die Probenoberfläche, die von der Zelle gesehen wird ist proportional zu $\cos \phi$ und damit auch der Teilchenfluß der Zelle durch die Probenoberfläche.

$$
N=\frac{\Gamma}{\pi r^{2}} \cdot C^{M o t} \cdot \cos \phi\left[m^{-2} s^{-1}\right]
$$


Tabelle B.1: Parameter für die Abschätzung der Teilchenflüsse der Ga und der Gd Zelle.

\begin{tabular}{|c|c|c|c|c|c|c|c|}
\hline Material & $M^{1}$ & $\Delta \mathrm{H}_{\text {vap }}{ }^{1}$ & $\mathrm{p}_{0}{ }^{1}$ & $\mathrm{~T}_{0}{ }^{1}$ & & & $\phi$ \\
\hline $\mathrm{Ga}$ & $69,72 \mathrm{~g} / \mathrm{mol}$ & $258,7 \mathrm{~kJ} / \mathrm{mol}$ & $101325 \mathrm{~Pa}$ & $2478 \mathrm{~K}$ & & & $33^{\circ}$ \\
\hline $\mathrm{Gd}$ & $157,25 \mathrm{~g} / \mathrm{mol}$ & $359,4 \mathrm{~kJ} / \mathrm{mol}$ & $101325 \mathrm{~Pa}$ & $3569 \mathrm{~K}$ & & & $33^{\circ}$ \\
\hline Tiegel & $A_{e}(a)$ & $b$ & r & $W_{a}$ & $W_{b}$ & $\mathrm{~L}_{0}$ & $d_{0}$ \\
\hline Ga Zelle & $267 \mathrm{~mm}^{2}$ & $195 \mathrm{~mm}^{2}$ & $273 \mathrm{~mm}$ & 0,219 & 1 & $88 \mathrm{~mm}$ & $18,5 \mathrm{~mm}$ \\
\hline Gd Zelle & $119 \mathrm{~mm}^{2}$ & $83 \mathrm{~mm}^{2}$ & $255 \mathrm{~mm}$ & 0,220 & 1 & $56,25 \mathrm{~mm}$ & $12,3 \mathrm{~mm}$ \\
\hline
\end{tabular}

\section{B.3 Verwendete Zellen und deren Flüsse}

In der Anlage ELSA (Abschnitt 2.1) kommen für die Bereitstellung von Ga und Gd Effusionszellen mit nahezu zylindrischen Tiegeln zum Einsatz. Da Näherungen für konische Tiegel deutlich anspruchsvoller sind und die Öffnungswinkel der Zellen mit unter $1^{\circ}$ sehr klein werden hier die Näherungen für zylindrische Zellen verwendet. Die Tatsache, daß die Zellen leicht konisch sind beeinflußt aber trotzdem die Motzfeldkorrektur, da die Flächen $a$ und $b$ unterschiedlich sind und damit der Fluchtkoeffizient (Gleichung B.4) beeinflußt wird.

Die theoretischen Teilchenflüsse der Zellen wurden mit Gleichung B.6 berechnet. Der von der Temperatur abhängige Gleichgewichtsdruck $p_{e q}$ in Gleichung B.2 wird durch die Clausius-Clapeyron-Gleichung beschrieben.

$$
p_{e q}=p_{0} \cdot e^{-\frac{\Delta H_{v a p}}{R}\left(\frac{1}{T}-\frac{1}{T_{0}}\right)}
$$

$\Delta H_{v a p}$ ist die Verdampfungsenthalpie, $p_{0}$ und $T_{0}$ ist ein Wertepaar der Dampfdruckkurve und $R$ ist die allgemeine Gaskonstante. Die verwendeten Werte für die einzelnen Größen sind in Tabelle B.1 aufgelistet. Die Fläche $a$ entspricht der Effusionsfläche $A_{e}$. Für die das Wertepaar der Dampfdruckkurve wird der Siedepunkt bei Normaldruck verwendet.

Abbildung B.2(a) zeigt die theoretischen Teilchenflüsse der Ga und der Gd Zelle auf der Probenoberfläche in Abhängigkeit von der Temperatur der Zellen. Besser zum Vergleich mit den Bedingungen in der Anlage und den gemessenen Werten der Partialdrücke in der Wachstumskammer der Anlage ist aber die Darstellung in Abbildung B.2(b). Hier wurde mit Hilfe des Zusammenhangs $\Gamma=\mathrm{N} \cdot \mathrm{A}_{\mathrm{e}}$ und Gleichung B.2 der erwartete Teilchenfluß in einen Partialdruck an der Stelle der Probe umgerechnet. Jetzt können die Partialdrücke der Zellen an der Position der Probenoberfläche mit dem Kammerdruck und BEP verglichen werden. In Abbildung B.2(b) ist zusätzlich eine Punktkalibrierung der Ga Zelle eingetragen. Der gemessene BEP liegt leicht unterhalb der theoretischen Kurve. 


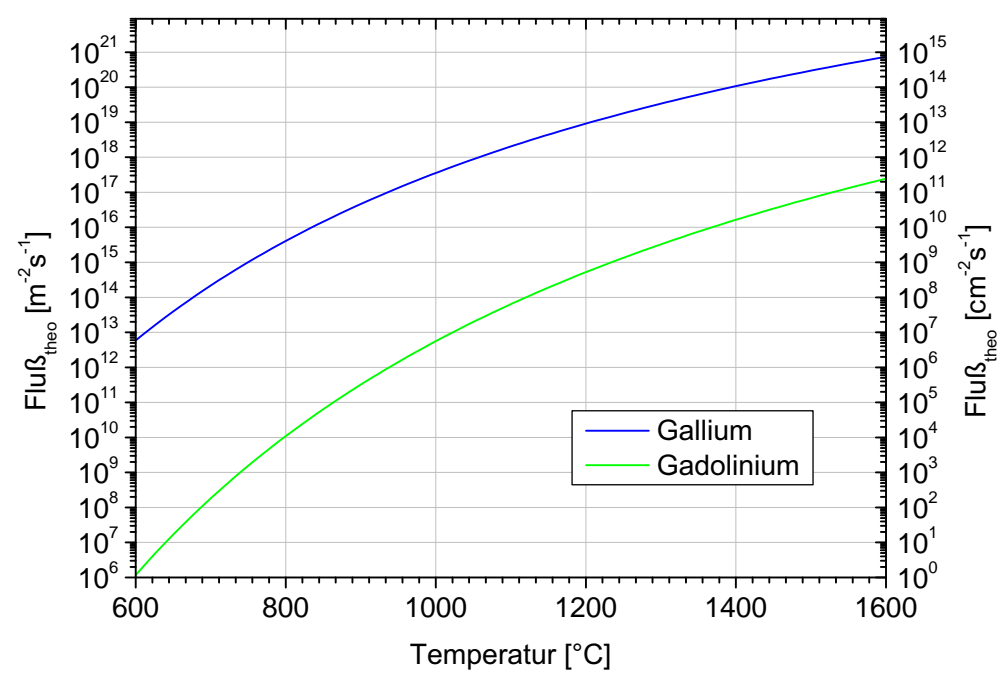

(a) Teilchenflüsse

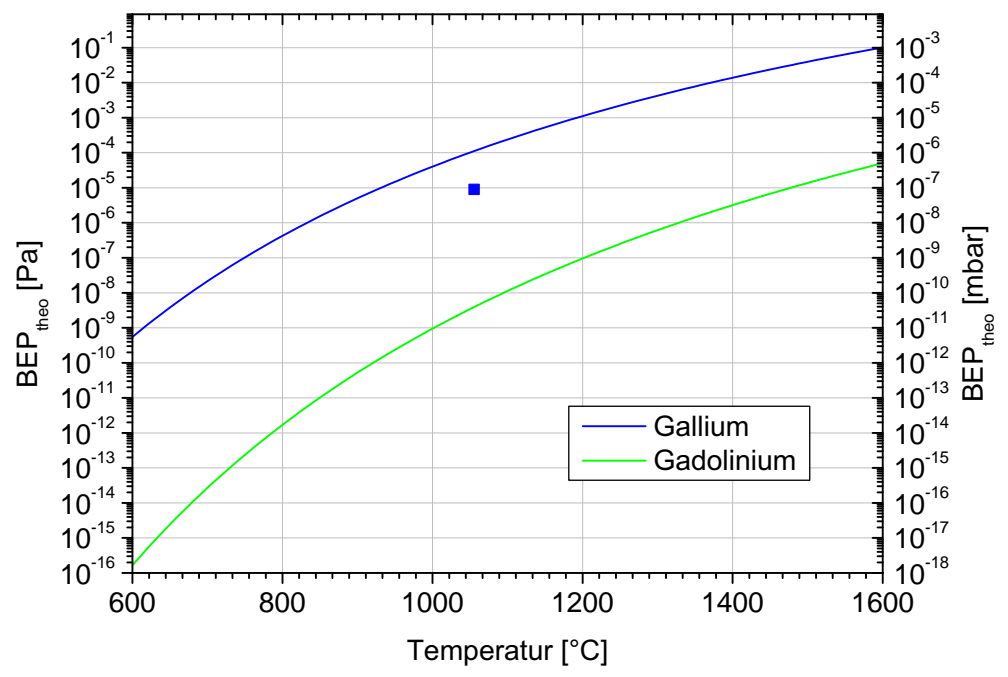

(b) BEP theoretisch

Abbildung B.2: Teilchenflüsse der Effusionszellen 


\section{Anhang C}

\section{Positronenvernichtung}

Die Postitronenvernichtungsspektroskopie (PAS - positron annihilation spectroscopy) ist eine mächtige Methode, um einen Kristall auf Defekte, insbesondere Leerstellen, zu charakerisieren. Abbildung C.1 zeigt die Skizze eines PAS-Experimentes mit einer ${ }^{22} \mathrm{Na}$ Quelle. Die Positronen aus der Quelle gelangen entweder beschleunigt als Positronenstrahl oder direkt in die zu analysierende Schicht. Die Positronen thermalisieren sehr schnell im Kristall (ca. 3 ps) und verhalten sich daher wie vollständig thermalisierte Teilchen im Festkörper. Typische Lebensdauern liegen bei ca. 150 - 300 ps. Die Entstehung des Positrons in der ${ }^{22} \mathrm{Na}$-Quelle wird von der Entstehung eines Photons mit einer Energie von $1,28 \mathrm{MeV}$ begleitet. Bei der Vernichtung des Positrons entstehen weitere Photonen mit einer Energie von $511 \mathrm{keV}$. Diese begleitende Strahlung wird als Start- bzw. Stopsignal für die Lebensdauerspektroskopie verwendet. Das Signal der Stop-Photonen ist gegenüber dem der Start-Photonen Doppler-verbreitert.

Analog zu freien Elektronen im Kristall haben auch Positronen Zustände an Leerstellen. Fehlt ein Atomrumpf im Kristall, so ist an diesem Ort die (Coulomb) Abstoßung, welche auf das Positron wirkt kleiner und ein lokalisierter Zustand für das Positron entsteht. Je größer das Leervolumen im Kristall ist, desto tiefer ist auch der potentialtopfartige Zustand. Bindungsenergien von $1 \mathrm{eV}$ und mehr sind üblich.

Auch negativ geladene Ionen können Positronen in relativ flachen Zuständen in ihrem Coulombfeld binden $(0,1 \mathrm{eV})$. Diese Defekte konkurrieren als bindende Zentren für die Positronen mit den Leerstellen und beeinflussen so die Lebensdauern, insbesondere die 


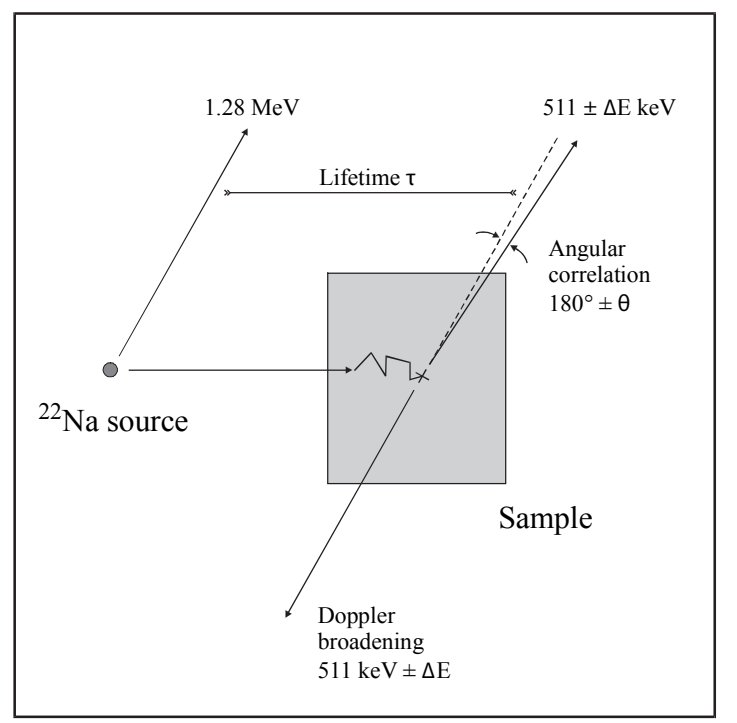

\section{Abbildung C.1:}

Die Skizze zeigt die Versuchsanordnung eines Positronenvernichtungsexperimentes mit einer ${ }^{22} \mathrm{Na}-$ Quelle. Entstehung und Vernichtung der Positrons werden von Photonen begleitet, die als Startund Stopsignal der Lebensdauerspektroskopie dienen. Die Winkelverteilung des Stop-Photons ist Doppler-verbreitert und enthält weitere Informationen über Defekte. Grafik entnommen aus [61].

mittlere Lebensdauer $\tau_{\mathrm{av}}$. Da diese Defekte allerdings kein Leervolumen besitzen, ist die Lebensdauer $\tau_{\text {ion }}$ des Zustandes nicht von dem des Volumenkristalles zu unterscheiden. Mit Hilfe von temperaturabhängigen Messungen der Lebensdauer kann aber der Einfluß von negativen Ionen auf die mittlere Lebensdauer $\tau_{\mathrm{av}}$ bestimmt werden, da mit zunehmender Temperatur die Positronen immer weniger an Ionen binden und somit deren Einfluß mit steigender Temperatur auch abnimmt. Negative Ionen haben damit eine unterschiedliche temperaturabhängige Charakteristik als die deutlich stärker bindenden Leerstellen ( $1 \mathrm{eV}$ und mehr).

\section{C.1 Lebensdauerspektroskopie}

Da in Leerstellen die Elektronendichte lokal erniedrigt ist, ist auch die Lebensdauer $\tau$ der Positronen an solchen Defekten erhöht, da die Wahrscheinlichkeit einer Vernichtung mit einem Elektron von der Elektronendichte abhängt. Dies wird bei der Lebensdauerspektroskopie ausgenutzt, um im Vergleich mit theoretischen Modellen die Defekte zu identifizieren und zu quantifizieren. Jeder Positronenzustand leistet einen exponentiellen Beitrag zur mittleren Lebensdauer $\tau_{\mathrm{av}}$ der Positronen. Damit schreibt sich die 
Wahrscheinlichkeit $\mathrm{n}(\mathrm{t})$ eines Positrons zur Zeit t lebendig zu sein wie folgt.

$$
-\frac{\mathrm{d} n(t)}{\mathrm{dt}}=\sum_{i} \frac{I_{i}}{\tau_{i}} \exp \left(t / \tau_{i}\right)
$$

Für jeden Beitrag können jetzt Intensität $I_{i}$ und Lebensdauer $\tau_{i}$ bestimmt werden. Je nach Meßbedingungen können so 2-3 Beiträge aufgelöst werden, wenn für deren Lebensdauern $\tau_{1} / \tau_{2}>1,5$ gilt.

Bei Messungen bei denen die einzelnen Komponenten nicht aufgelößt werden können, kann trotzdem mit Hilfe der mittleren Lebensdauer $\tau_{\mathrm{av}}$ eine Aussage darüber gemacht werden, ob Defekte vorliegen oder der Kristall frei von (detektierbaren) Defekten ist, da selbst kleine Änderungen der mittleren Lebensdauer von wenigen Pikosekunden eine signifikante Änderung darstellen.

Die typische Lebensdauer für den GaN Volumenkristall und Materialien mit ähnlicher atomarer Dichte (z.B. Al) ist 165 ps [61]. Ga Leerstellen in GaN zeigen eine Lebensdauer von 235 ps. Stickstoff Leerstellen haben theoretische Lebensdauern nahe der des Volumenkristalls, da sie ein sehr kleines Leervolumen haben. Experimentell sind sie somit schwer nachzuweisen. Darüber hinaus sind Stickstoff Leerstellen für gewöhnlich positiv geladen und damit aufgrund der Coulombabstoßung keine effizienten Positronenfallen.

\section{C.2 Dopplerverbreiterungsspektroskopie}

Das Elektronen mit dem sich das Positron vernichtet hat entlang der Richtung der $511 \mathrm{keV}$ Begleitstrahlung eine Impulskomponente, welche eine Dopplerverbreiterung dieser Linie verursacht und damit die (eindimensionale) Impulsverteilung des Elektrons abbildet. Nach einigen Auswertungsschritten (siehe z.B. [61]) erhält man die entsprechende Impulsverteilungskurve der Elektronen. Diese Kurve wird mit zwei Parametern (S und W) beschrieben. ${ }^{1}$

$\mathrm{S}$ ist der sog. low momentum parameter und representiert somit hauptsächlich Elektronen mit niedrigem Impuls, also die Valenzelektronen. Kernelektronen haben einen höheren Impuls und werden durch den high momentum parameter $\mathrm{W}$ representiert. Mit Hilfe dieser Parameter können so bei der Dopplerverbreiterungsspektroskopie Aussagen über die Art und Konzentration der Defekte gemacht werden und auch einfach verschiedene Proben miteinander verglichen werden.

\footnotetext{
${ }^{1}$ Für eine genaue Definition der Parameter S und W siehe z.B. [61], die Erkenntnisse, die aus ihnen werden in Abschnitt 5.3 diskutiert.
} 


\section{Anhang D}

\section{Kalibrierung der Oberflächentemperatur}

Ziel der Kalibrierung ist die Überprüfung der alten, vorliegenden Kalibrierung der Heizertemperatur im Episoft. Des weiteren werden Unterschiede in der Oberflächentemperatur von den unterschiedlichen Substraten Si, Saphir/GaN Substraten und SiC untersucht. Die Messungen werden mit einem Pyrometer durchgeführt. Zur Kalibrierung der absoluten Temperaturskala werden die Schmelzpunkte von Aluminium und Silber verwendet. 
Die Temperaturkalibrierung des Probenhalters in der MBE-Anlage ELSA wurde noch zu Jülicher Zeiten vorgenommen und von dortan als gegeben angesehen. Da aber einige Parameter geändert wurden soll diese Kalibrierung jetzt überprüft werden. Als die gavierensten Änderungen seit der alten Kalibrierung sind der Wechsel der Haltemaske, (um von der Probengeometrie 14x7 $\mathrm{mm}^{2}$ auf $10 \times 10 \mathrm{~mm}^{2}$ umzustellen) und der Wechsel auf Saphir/GaN Substrate statt Si zu nennen. Es wurden auch kleinere Änderungen, wie ein neues Netzteil und dementsprechend ein neues p-loop und eine Änderung in der Verkabelung von Netzteil und Episoft Frame vorgenommen. Wie sich im Laufe der Kalibrierung herrausstellte ist auch die Rauhigkeit der verwendeten Grundplatte von großer Bedeutung.

\section{D.1 Der Probenhalter}

Der Probenheizer wird mit einem über ein Episoft p-loop gesteuertem Netzteil betrieben. Das Netzteil wird im constant current mode betrieben, um immer die gleiche Leistung auf dem Heizer zu haben, auch wenn Probleme mit Kontaktwiderständen am Heizer oder dem Bajonetteverschluß bestehen. Dabei wird die im p-loop eingestellte Temperatur über die Funktion

$$
I=A+B \cdot T+C \cdot T^{2}
$$

- $\mathrm{A}=-0,292$

- $\mathrm{B}=0,003549$

- $\mathrm{C}=2,5227 \cdot 10^{-6}$

in den entsprechenden Strom umgerechnet. Alle genannten Stellen sind signifikant. Der Heizer sollte nicht für lange Zeiten über $5 \mathrm{~A}$ betrieben werden.

Die genaue Einstellung des p-loops ist vom verwendeten Netzteil abhängig. Die Steuerung sollte auf eine Genauigkeit von zwei Nachkommastellen im resultierenden Strom am Heizer vorgenommen werden.

\section{D.2 Pyrometer}

Als Pyrometer wird ein Ircon Modline3 3V-12C05 mit einer P2 Linse verwendet. Die Emissivität wird entsprechend des Substrates gewählt und im jeweiligen Abschnitt beschrieben. Die Transmissivität des Fensters (Fenstermaterial: Kodial) wird mit einem Faktor 0,9 berücksichtig.

Ein Problem bei der Messung mit Pyrometern ist, daß nicht alle Materialien in einem bestimmten Wellenlängenbereich messbar sind. Si und SiC sind für den Messbereich des 
Pyrometers (900-970 nm) geeignet, Saphir und GaN sind allerdings in diesem Bereich durchsichtig und somit wird auch in sehr großem Anteil die Rückplatte des Probenhalters vom Pyrometer gesehen. Bei Saphir/GaN Substraten ist also keine verläßliche Temperaturbestimmung mit diesem Pyrometer machbar, da die Rückplatte unter dem Substrat gemessen wird.

Die Pyrometermessung ist extrem empfindlich gegenüber Fremdeinstrahlungen in die Optik oder auf Durchstrahlungen des zu messenden Werkstückes. Ist zwar die Methode ansich bis auf mehrere Nachkommastellen genau, so können durch solche systematischen Fehler leicht Abweichungen von $100^{\circ} \mathrm{C}$ auftreten.

\section{D.3 Nachvollziehen der alten Kalibrierung}

\section{D.3.1 Si Substrat}

Als erster Schritt bei der neuen Temperaturkalibrierung des Probenhalters wird die alte Kalibrierung nachvollzogen. Dabei wird auch Si als Substrat und die alte Haltemaske verwendet (Abb. D.1).

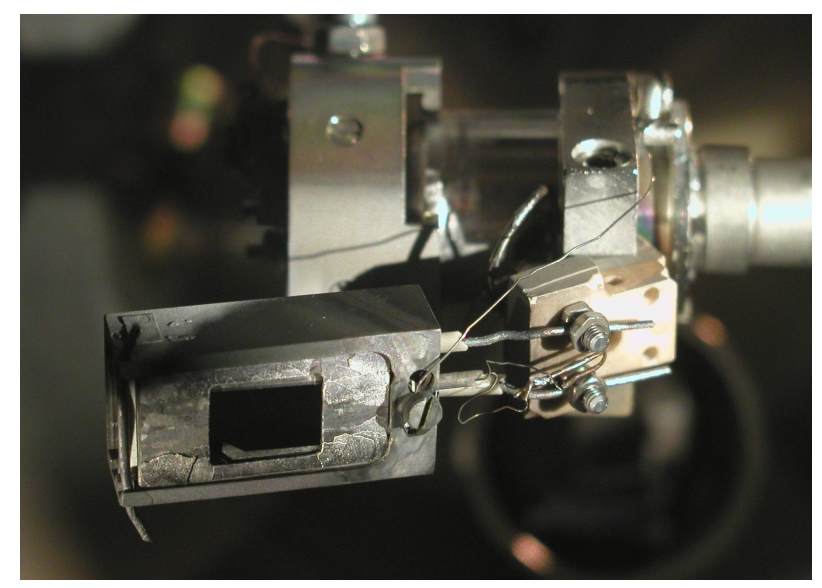

Abbildung D.1: Probenhalter mit alter Maske und Si Substrat

Da nicht bekannt ist welche Emissivität bei der alten Kalibrierung verwendet wurde, wurden für Si Substrat zwei verschiedene Kurven aufgenommen (Tabelle D.1). Die in der Literatur gefundene Emissivität von 0,68 (mit Faktor 0,9 für das Fenster ergibt sich $0,612)$ und die Emissivität, die bei einem $\mathrm{T}_{\text {set }}=550^{\circ} \mathrm{C}$ auch ein $\mathrm{T}_{\text {pyro }}=550^{\circ} \mathrm{C}$ liefert $(0,43)$.

Abbildung D.2 zeigt die Temperaturkurven des Probenhalters mit der alten Maske und einem Si Substrat. Dabei ist die Pyrometertemperatur gegen die Solltemperatur 


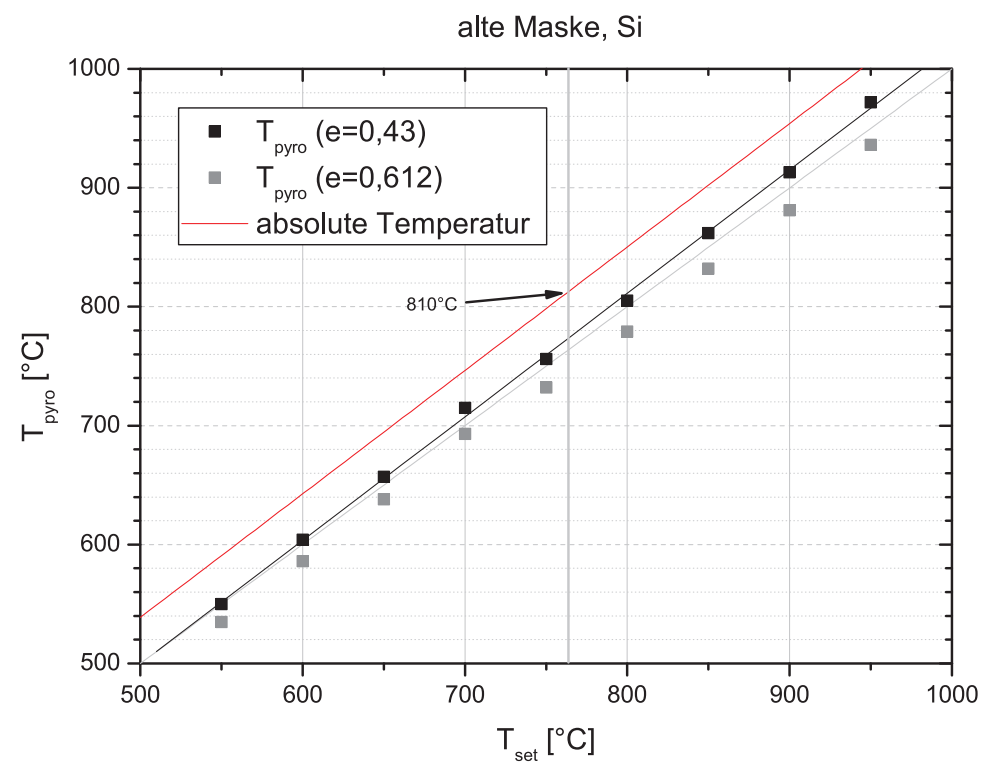

Abbildung D.2:

Temperaturkurven der alten Maske mit Si Substrat, Messwerte siehe Abschnitt D.5, Tabelle D.1 und D.2.

aufgetragen. Die Winkelhalbierende stellt eine korekte Kalibrierung dar. Weder bei einer Emissivität von 0,612, noch von 0,43 wird diese erreicht. Die absolute Temperatur wurde aus der Messung der neuen Maske mit einem Si Substrat gewonnen und wird dort beschrieben (Abschnitt D.4.1). Es ist zu bemerken, daß die absolute Temperatur deutlich höher als die Kalibrierungskurve liegt. Die vertikale graue Linie markiert die Standardwachstumstemperatur in der Anlage. Bei Si entspricht eine Solltemperatur von $760^{\circ} \mathrm{C}$ einer absoluten Temperatur von $810^{\circ} \mathrm{C}$. Zusammen mit den Ergebnissen aus Abschnitt D.4.3 läßt das die Vermutung zu, daß die alte Kalibrierung mit einem SiC Substrat und nicht mit Si gemacht wurde.

\section{D.3.2 Saphir/GaN Substrat}

Um später Vergleiche zu der neuen Haltemaske machen zu können und so den Einfluß der Probengeometrie auf die Oberflächentemperatur abschätzen zu können wurde auch die alte Make mit dem neuen Saphir/GaN Substrat vermessen. Dabei wurde für das Pyrometer aufgrund der in Abschnitt D.2 genannten Probleme eine Emissivität von $\epsilon=1$ gewählt. Zusätzlich wurde für eine absolute Kalibrierung der Temperaturskala ein Stück Al Draht mit Leitsilber auf die Substratoberfläche geklebt.

Die Abbildung D.3 zeigt die Temperaturkurven dieser Messung. Zusätzlich ist der Schmelzpunkt von $\mathrm{Al}$ als roter Punkt eingetragen. Die absolute Temperaturkurve wurde 


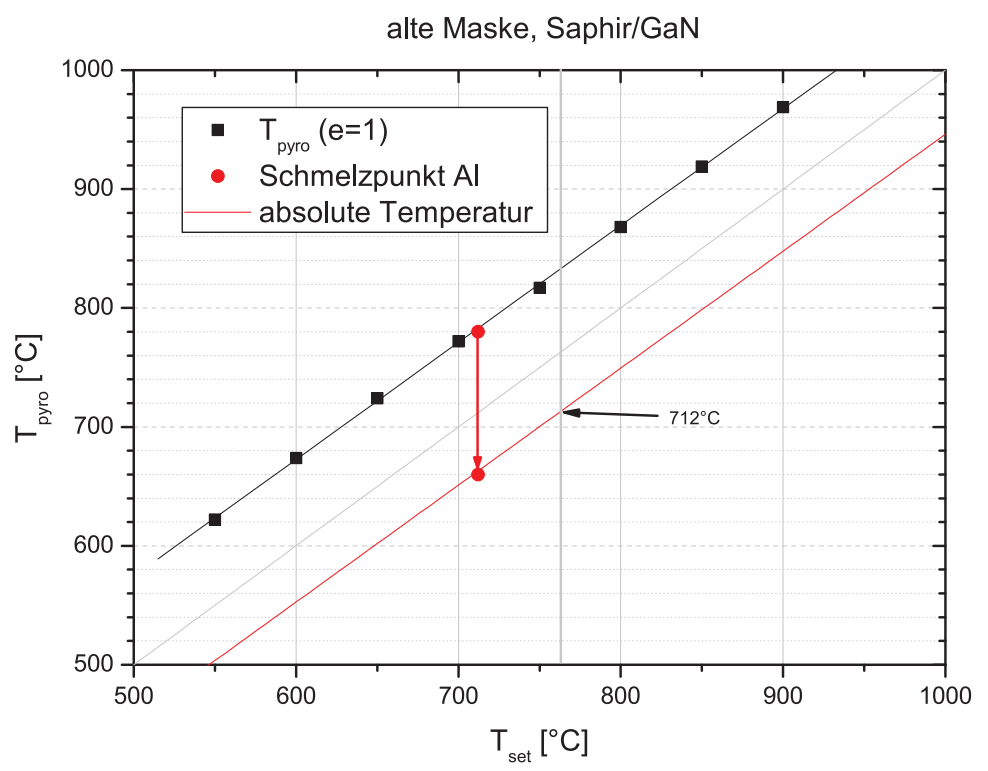

\section{Abbildung D.3:}

Temperaturkurven der alten Maske mit Saphir/GaN Substrat, Messwerte siehe Abschnitt D.5,

Tabellen D.1 und D.2.

durch Verschieben des Fits durch die Meßpunkte auf die Schmelztemperatur von $\mathrm{Al}$ erreicht $\left(\mathrm{T}_{\mathrm{Al}}=660^{\circ} \mathrm{C}\right)$.

Es ist deutlich zu erkennen, daß die absolute Temperatur deutlich niedriger liegt als bei Si Substrat, was auf die schlechtere Wärmeleitfähigkeit von Saphir zurückzuführen ist.

\section{D.4 Neue Maske}

Das Hauptziel der Kalibrierung ist die Bestimmung der absoluten Oberflächentemperatur verschiedener Substrate bei gleicher Solltemperatur. Dazu wurden jeweils die Substrate Si, Saphir/GaN und SiC mit einem Al und einem Ag Stück beklebt (Ausnahme: Si Substrat) und dann die Pyrometertemperatur in Abhängigkeit von der Solltemperatur gemessen.

\section{D.4.1 Si Substrat}

Abbildung D.4 zeigt die Temperaturkurven der Messung an Si Substrat mit der neuen Haltemaske. Es wurde hier nur die Emissivität von 0,43 gemessen, da diese bei der Messung mit der alten Maske die besseren Ergebnisse gezeigt hat. Die Messung paßst 


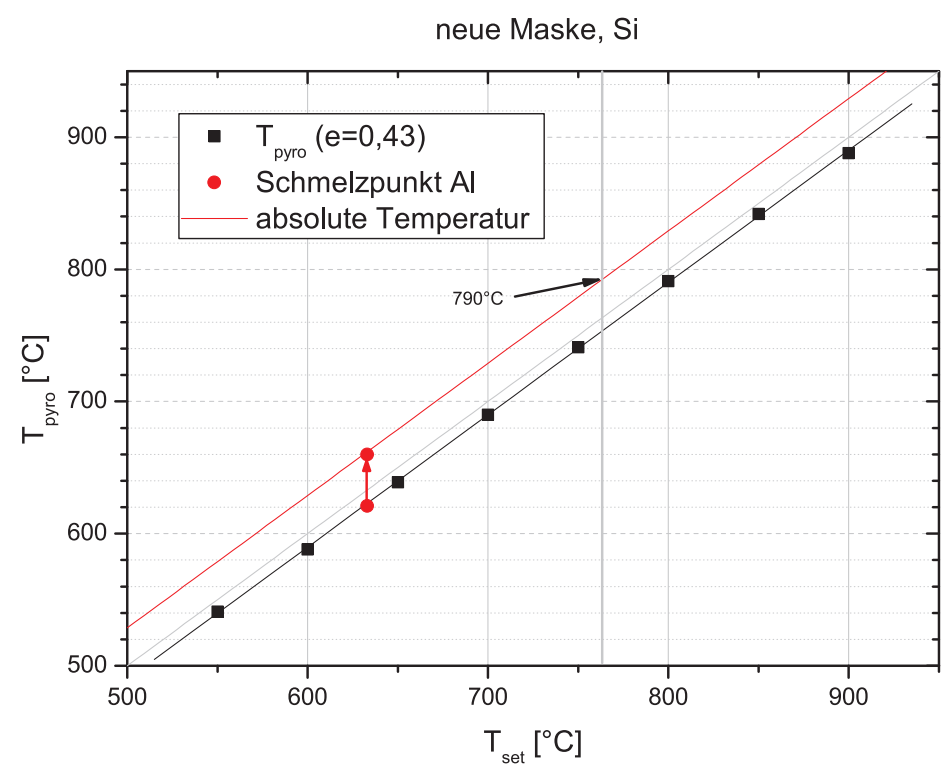

Abbildung D.4:

Temperaturkurven der neuen Maske mit Si Substrat, Messwerte siehe Abschnitt D.5, Tabellen D.3 und D.2.

gut zur Messung des Si Substrates mit der alten Maske (Abbildung D.2) und zeigt, daß die Oberflächentemperatur mit der neuen Maske ca. $20^{\circ} \mathrm{C}$ kälter ist als mit der alten Maske. Die absolute Temperaturskale in Abbildung D.2 wurde dadurch erzeugt, daß der Fit um die gleiche Temperaturdifferenz wie in Abbildung D.4 nach oben verschoben wurde. Diese Vorgehensweise ist natürlich nur eine Näherung, ist aber hier der einzige Anhaltspunkt auf die absolute Temperaturskala des Si mit alter Maske.

\section{D.4.2 Saphir/GaN Substrat}

Abbildung D.6 zeigt die Messung des Saphir/GaN Substrates mit der neuen Maske. Die Messung unterscheidet sich stark von der vergleichbaren Messung mit alter Maske (Abbildung D.3). Diese großen Abweichungen von der Solltemperatur erschienen ungewöhnlich hoch und deshalb wurde nach einem möglichen systematischen Fehler gesucht. Ein untersuchter Punkt war dabei die Rückplatte des Probenhalters (Tantalplatte direkt unter dem Substrat). Diese stammte aus Jülicher Zeiten und mußte gerade vor dieser Messung erneuert werden, da sich das Stück Silizium der vorherigen Messung auf der Platte festgebrannt hatte.

Die neue Rückplatte erschien deutlich rauher, als die alte aus Jülicher Zeiten. Um den Einfluß der Rauhigkeit der Rückplatte zu untersuchen wurde diese mit $15 \mu \mathrm{m}$ Schleifpaste poliert. Abbildung D.5(a) und D.5(b) zeigen die Rückplatte im unpolierten und 


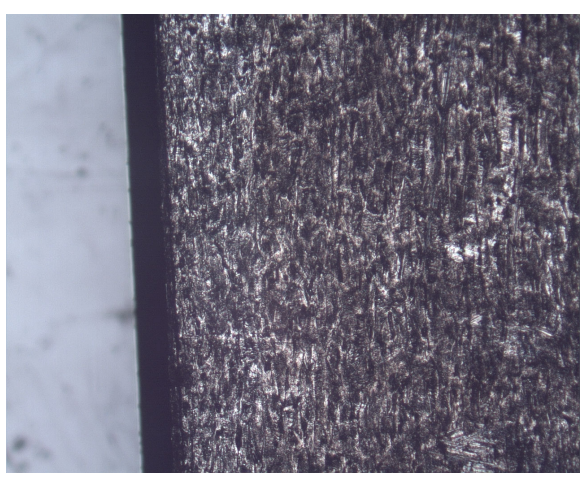

(a) unpoliert

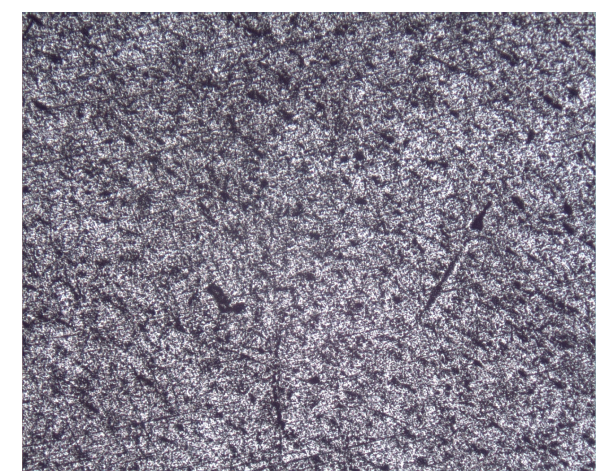

(b) poliert

\begin{abstract}
Abbildung D.5:
Vergleich der Rückplatte im optischen Mikroskop. Die Skala der beiden Bilder ist die gleiche. a) zeigt die Platte im Ausgangszustand und b) nach einer Politur mit einer $15 \mu \mathrm{m}$ Schleifpaste.
\end{abstract}

im polierten Zustand. Es ist zu erkennen, daß nach der Politur die Platte eine deutlich homogenere Struktur aufweißt.

Die Wiederholung der Messung mit polierter Platte ist in Abbildung D.7 zu sehen. Auswirkungen auf die Emissivität der Rückplatte durch die Politur sind nur leicht zu erkennen. Die mit dem Pyrometer aufgenommene Kurve bei der Messungen mit polierter Platte liegt ca. $10^{\circ} \mathrm{C}$ höher als vor der Politur. Unterschiede in den Messungen sind bei hohen Solltemperaturen zu erkennen. Nach der Politur scheint der Heizer ab einer Solltemperatur von ca. $1000^{\circ} \mathrm{C}$ in eine Art Sättigungsverhalten überzugehen. Wahrscheinlich ist das Abstrahlverhalten des jetzt deutlich wärmeren Substrates dafür verantwortlich.

Ein gravierender Unterschied ist in der absoluten Temperaturkurve zu erkennen. Wo es bei der Messung mit unpolierter Platte nicht möglich war bei einer Solltemperatur von $1080^{\circ} \mathrm{C}$ das Silberstück nicht zu schmelzen, kann nach der Politur bei einer Solltemperatur von $1035^{\circ} \mathrm{C}$ der Schmelzpunkt von Silber gefunden werden (vgl. Abbildung D.6 und D.7). Vergleicht man die absoluten Temperaturen bei der standard Wachstumstemperatur von $760^{\circ} \mathrm{C}$, so findet man eine um ca. $90^{\circ} \mathrm{C}$ höhere Temperatur nach der Politur.

Vergleicht man jetzt die Messung von Saphir/GaN Substrat mit neuer und alter Maske (Abbildungen D.3 und D.7), so stellt man fest, daß die absolute Temperaturskala zwar eine etwas unterschiedliche Steigung hat, sie aber bei der Solltemperatur von $760^{\circ} \mathrm{C}$ beide den nahezu gleichen Wert von ca. $715^{\circ} \mathrm{C}$ aufweisen. Ein Einfluß der Maske auf die Standardbedingungen für das GaN Wachstum auf Saphir/GaN Substraten ist also nicht erkennbar. Bei von der Standardtemperatur von $760^{\circ} \mathrm{C}$ abweichenden Temperaturen 
können Unterschiede auftreten.

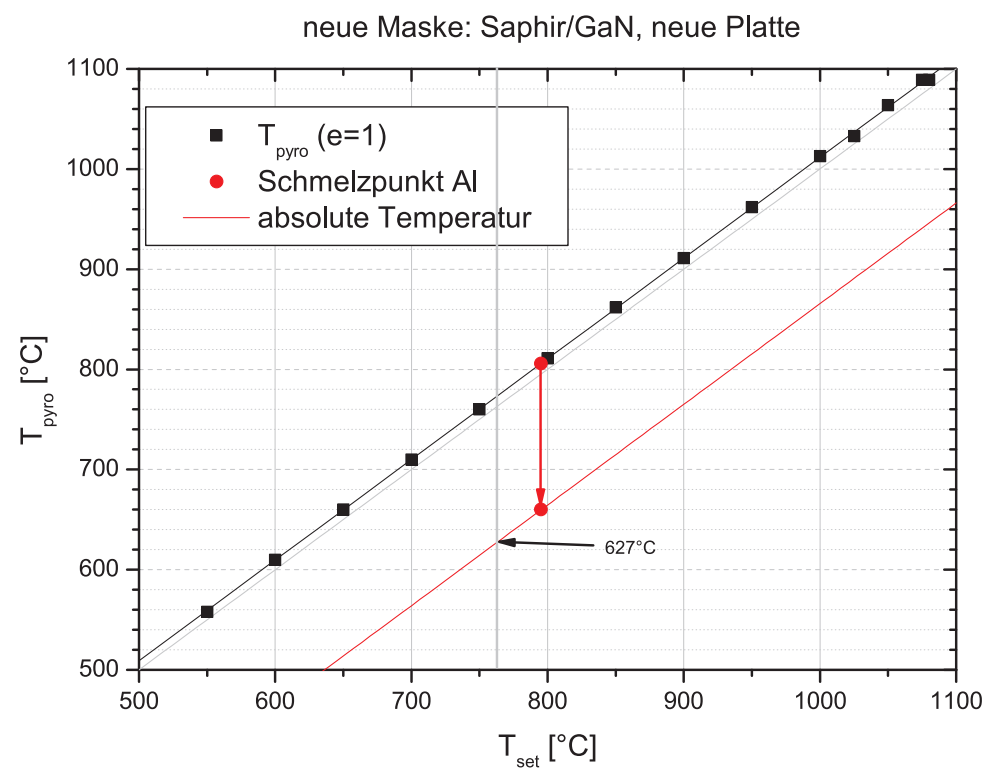

\section{Abbildung D.6:}

Temperaturkurven der neuen Maske mit Saphir/GaN Substrat auf der neuen Rückplatte, Messwerte siehe Abschnitt D.5, Tabellen D.3 und D.2.

\section{D.4.3 SiC Substrat}

Als letztes gängiges Substrat wurde $\mathrm{SiC}$ getestet. Für $\mathrm{SiC}$ findet man eine Emissivität von 0,85 in der Literatur. Zusammen mit dem Faktor 0,9 für das Fenster ergibt sich dann der verwendete Wert von 0,765 .

In Abbildung D.8 sind die entsprechenden Kurven zu sehen. SiC zeigt bei allen Kurven die beste Übereinstimmung mit der Solltemperatur. Die absolute Temperatur bei standard Wachstumstemperatur von $760^{\circ} \mathrm{C}$ beträgt knapp $750^{\circ} \mathrm{C}$ und liegt damit $30-35^{\circ} \mathrm{C}$ höher als auf Saphir/GaN Substraten. 


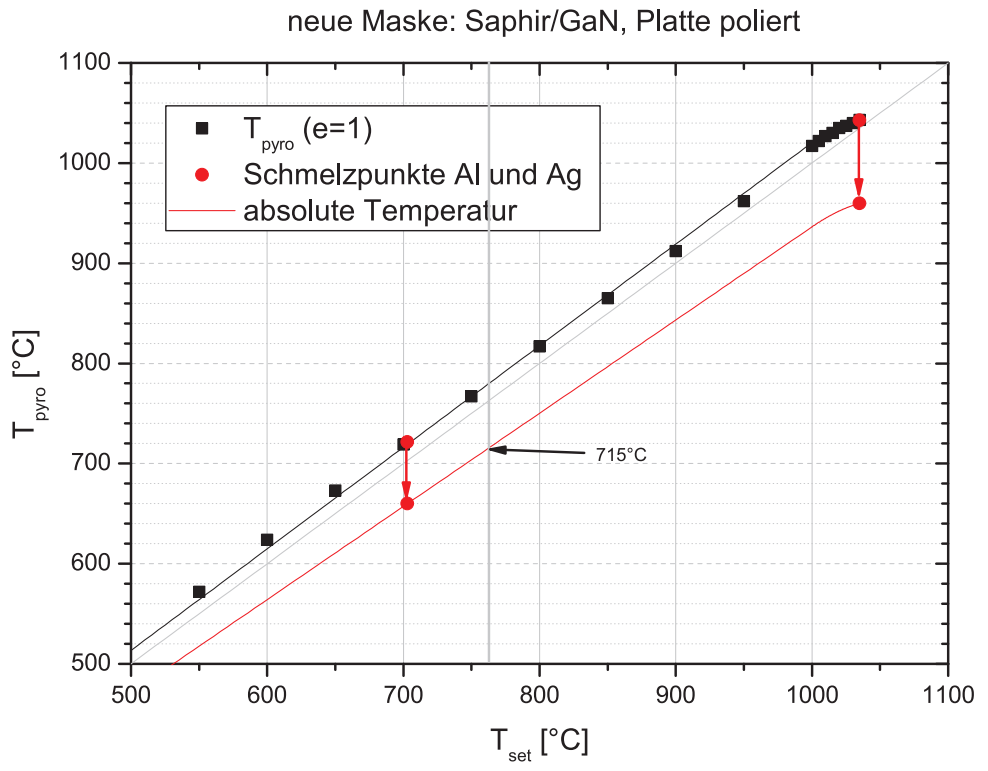

\section{Abbildung D.7:}

Temperaturkurven der neuen Maske mit Saphir/GaN Substrat auf der neuen Rückplatte, die Platte wurde vor der Messung mit $15 \mu \mathrm{m}$ Schleifpaste poliert, Messwerte siehe Abschnitt D.5, Tabellen D. 3 und D.2.

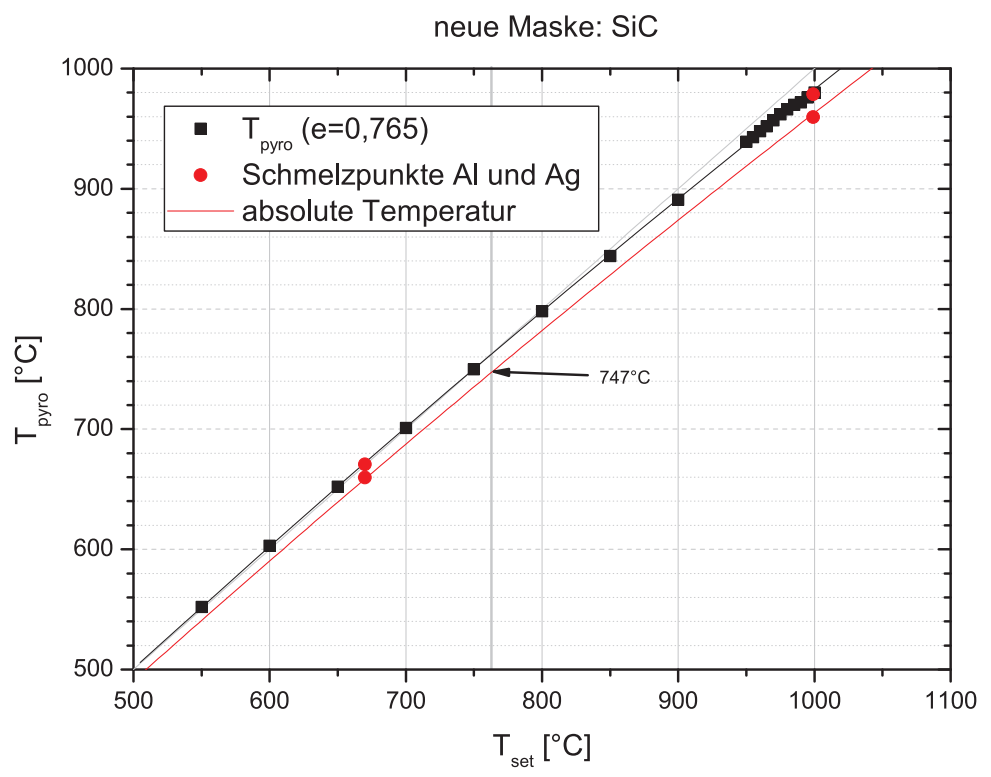

\section{Abbildung D.8:}

Temperaturkurven der neuen Maske mit SiC Substrat, Messwerte siehe Abschnitt D.5, Tabellen D. 3 und D.2. 


\section{D.5 Messtabellen}

Tabelle D.1: alte Maske

\begin{tabular}{cccc}
\hline \hline & \multicolumn{2}{c}{ Si Substrat } & Saphir/GaN \\
$\mathrm{T}_{\text {set }}\left[{ }^{\circ} \mathrm{C}\right]$ & \multicolumn{2}{c}{$\mathrm{T}_{\text {pyro }}\left[{ }^{\circ} \mathrm{C}\right]$} & $\mathrm{T}_{\text {pyro }}\left[{ }^{\circ} \mathrm{C}\right]$ \\
& $\epsilon=0,612$ & $\epsilon=0,43$ & $\epsilon=1$ \\
\hline 550 & 535 & 550 & 622 \\
600 & 586 & 604 & 674 \\
650 & 638 & 657 & 724 \\
700 & 693 & 715 & 772 \\
750 & 732 & 756 & 817 \\
800 & 779 & 805 & 868 \\
850 & 832 & 862 & 919 \\
900 & 881 & 913 & 969 \\
950 & 936 & 972 & \\
\hline
\end{tabular}

Tabelle D.2: Schmelzpunkte

\begin{tabular}{lcccccccc}
\hline \hline & & \multicolumn{3}{c}{ Si } & \multicolumn{3}{c}{ Saphir/GaN } & \multicolumn{2}{c}{ SiC } \\
& & $\mathrm{Al}$ & $\mathrm{Ag}$ & $\mathrm{Al}$ & $\mathrm{Ag}$ & $\mathrm{Al}$ & $\mathrm{Ag}$ \\
\hline \multirow{2}{*}{ alte Maske } & $\mathrm{T}_{\text {set }}\left[{ }^{\circ} \mathrm{C}\right]$ & - & - & 713 & - & - & - \\
& $\mathrm{T}_{\text {pyro }}\left[{ }^{\circ} \mathrm{C}\right]$ & - & - & 780 & - & - & - \\
& & & & & & & \\
\hline \multirow{2}{*}{ neue Maske } & $\mathrm{T}_{\text {set }}\left[{ }^{\circ} \mathrm{C}\right]$ & 633 & - & 703 & 1035 & 670 & 999 \\
& $\mathrm{~T}_{\text {pyro }}\left[{ }^{\circ} \mathrm{C}\right]$ & 621 & - & 722 & 1043 & 671 & 979
\end{tabular}

Literatur $\quad \mathrm{Al}: \mathrm{T}_{\text {Schmelz }}=660^{\circ} \mathrm{C} \quad \mathrm{Ag}: \mathrm{T}_{\text {Schmelz }}=960^{\circ} \mathrm{C}$


Tabelle D.3: neue Maske

\begin{tabular}{|c|c|c|c|c|}
\hline \multirow[t]{2}{*}{$\mathrm{T}_{\text {set }}\left[{ }^{\circ} \mathrm{C}\right]$} & \multirow[t]{2}{*}{$\begin{array}{c}\text { Si Substrat } \\
\mathrm{T}_{\text {pyro }}\left[{ }^{\circ} \mathrm{C}\right] \\
\epsilon=0,432\end{array}$} & \multicolumn{2}{|c|}{$\begin{array}{c}\text { Saphir/GaN } \\
\mathrm{T}_{\text {pyro }}\left[{ }^{\circ} \mathrm{C}\right] \\
\epsilon=1\end{array}$} & \multirow[t]{2}{*}{$\begin{array}{c}\mathrm{SiC} \\
\mathrm{T}_{\text {pyro }}\left[{ }^{\circ} \mathrm{C}\right. \\
\epsilon=0,765\end{array}$} \\
\hline & & unpoliert & poliert & \\
\hline 550 & 541 & 558 & 577 & 552 \\
\hline 600 & 588 & 610 & 624 & 603 \\
\hline 650 & 639 & 660 & 673 & 652 \\
\hline 700 & 690 & 710 & 719 & 701 \\
\hline 750 & 741 & 760 & 767 & 750 \\
\hline 800 & 791 & 811 & 817 & 798 \\
\hline 850 & 842 & 862 & 865 & 844 \\
\hline 900 & 888 & 911 & 912 & 891 \\
\hline 950 & & 962 & 962 & 939 \\
\hline 955 & & & & 943 \\
\hline 960 & & & & 948 \\
\hline 965 & & & & 952 \\
\hline 970 & & & & 957 \\
\hline 975 & & & & 962 \\
\hline 980 & & & & 966 \\
\hline 985 & & & & 970 \\
\hline 990 & & & & 972 \\
\hline 995 & & & & 976 \\
\hline 1000 & & 1013 & 1017 & \\
\hline 1005 & & & 1022 & \\
\hline 1010 & & & 1027 & \\
\hline 1015 & & & 1030 & \\
\hline 1020 & & & 1035 & \\
\hline 1025 & & 1033 & 1037 & \\
\hline 1030 & & & 1040 & \\
\hline 1035 & & & 1043 & \\
\hline 1050 & & 1064 & & \\
\hline 1075 & & 1089 & & \\
\hline 1080 & & 1089 & & \\
\hline
\end{tabular}




\section{Literaturverzeichnis}

[1] M. N. Baibich, J. M. Broto, A. Fert, F. N. Van Dau, F. Petroff, P. Etienne, G. Creuzet, A. Friederich, J. Chazelas, Giant Magnetoresistance of (001)Fe/(001) Cr Magnetic Superlattices, Phys. Rev. Lett. 61(21), 2472, 1988

[2] G. Binasch, P. Grünberg, F. Saurenbach, W. Zinn, Enhanced magnetoresistance in layered magnetic structures with antiferromagnetic interlayer exchange, Phys. Rev. B 39(7), 4828, 1989

[3] A. Zunger, S. Lany, H. Raebiger, The quest for dilute ferromagnetism in semiconductors: Guides and misguides by theory, Physics 3, 53, 2010

[4] T. Fukumura, H. Toyosaki, Y. Yamada, Magnetic oxide semiconductors, Semiconductor Science and Technology 20(4), S103, 2005

[5] N. Teraguchi, A. Suzuki, Y. Nanishi, Y.-K. Zhou, M. Hashimoto, H. Asahi, Room-temperature observation of ferromagnetism in diluted magnetic semiconductor GaGdN grown by RF-molecular beam epitaxy, Solid State Communications 122(12), 651, 2002

[6] H. Asahi, Y. K. Zhou, M. Hashimoto, M. S. Kim, X. J. Li, S. Emura, S. Hasegawa, GaN-based magnetic semiconductors for nanospintronics, Journal of Physics: Condensed Matter 16(48), S5555, 2004

[7] Y. K. Zhou, M. S. Kim, X. J. Li, S. Kimura, A. Kaneta, Y. Kawakami, S. Fujita, S. Emura, S. Hasegawa, H. Asahi, Optical properties of GaN-based magnetic semiconductors, Journal of Physics: Condensed Matter 16(48), S5743, 2004

[8] S. Dhar, O. Brandt, M. Ramsteiner, V. F. Sapega, K. H. Ploog, Colossal Magnetic Moment of Gd in GaN, Phys. Rev. Lett. 94(3), 037205, 2005

[9] J. K. Lang, Y. Baer, P. A. Cox, Study of the $4 f$ and valence band density of states in rare-earth metals. II. Experiment and results, Journal of Physics F: Metal Physics 11(1), 121, 1981 
[10] U. Vetter, Lanthanide Doped Wide Band Gap Semiconductors: Intra-4f Luminescence and Lattice Location Studies, Ph.D. thesis, Georg-August-Universität Göttingen, 2003

[11] K. Sato, L. Bergqvist, J. Kudrnovský, P. H. Dederichs, O. Eriksson, I. Turek, B. Sanyal, G. Bouzerar, H. Katayama-Yoshida, V. A. Dinh, T. Fukushima, H. Kizaki, R. Zeller, First-principles theory of dilute magnetic semiconductors, Rev. Mod. Phys. 82(2), 1633, 2010

[12] B. L. Sheu, R. C. Myers, J.-M. Tang, N. Samarth, D. D. Awschalom, P. Schiffer, M. E. Flatté, Onset of Ferromagnetism in Low-Doped $\mathrm{Ga}_{1-\mathrm{x}} \mathrm{Mn}_{\mathrm{x}} \mathrm{As}$, Phys. Rev. Lett. 99(22), 227205, 2007

[13] S. C. Erwin, A. G. Petukhov, Self-Compensation in Manganese-Doped Ferromagnetic Semiconductors, Phys. Rev. Lett. 89(22), 227201, 2002

[14] D.-D. Mai, Ferromagnetismus bei Raumtemperatur in mehrphasigen (Ga,Mn)N Schichten und Heterostrukturen, Ph.D. thesis, Georg-August-Universität Göttingen, 2009

[15] A. Bonanni, Ferromagnetic nitride-based semiconductors doped with transition metals and rare earths, Semiconductor Science and Technology 22(9), R41, 2007

[16] S. B. Ogale, R. J. Choudhary, J. P. Buban, S. E. Lofland, S. R. Shinde, S. N. Kale, V. N. Kulkarni, J. Higgins, C. Lanci, J. R. Simpson, N. D. Browning, S. Das Sarma, H. D. Drew, R. L. Greene, T. Venkatesan, High Temperature Ferromagnetism with a Giant Magnetic Moment in Transparent Co-doped SnO2 - $\delta$, Phys. Rev. Lett. 91(7), 077205, 2003

[17] S. Dhar, L. Pérez, O. Brandt, A. Trampert, K. H. Ploog, J. Keller, B. Beschoten, Gd-doped GaN: A very dilute ferromagnetic semiconductor with a Curie temperature above 300 K, Physical Review B 72(24), 245203, 2005

[18] J. Hite, R. Frazier, R. Davies, G. Thaler, C. Abernathy, S. Pearton, J. Zavada, E. Brown, U. Hömmerich, Effect of Si Co Doping on Ferromagnetic Properties of GaGdN, Journal of Electronic Materials 36(4), 391, 2007

[19] M. Roever, D.-D. Mai, A. Bedoya-Pinto, J. Malindretos, A. Rizzi, Electron stabilized ferromagnetism in GaGdN, physica status solidi (c) 5(6), 2352, 2008

[20] A. Ney, T. Kammermeier, E. Manuel, V. Ney, S. Dhar, K. H. Ploog, F. Wilhelm, A. Rogalev, Element specific investigations of the structural and magnetic properties of Gd:GaN, Applied Physics Letters 90(25), 252515, 2007 
[21] G. Martínez-Criado, O. Sancho-Juan, N. Garro, J. A. Sans, A. Cantarero, J. Susini, M. Roever, D.-D. Mai, A. Bedoya-Pinto, J. Malindretos, A. Rizzi, X-ray absorption in GaGdN: A study of local structure, Applied Physics Letters 93(2), 021916, 2008

[22] Y. Zhou, S. Choi, S. Kimura, S. Emura, S. Hasegawa, H. Asahi, High Gd Concentration GaGdN Grown at Low Temperatures, Journal of Superconductivity and Novel Magnetism 20(6), 429, 2007

[23] Y. K. Zhou, S. W. Choi, S. Emura, S. Hasegawa, H. Asahi, Large magnetization in high Gd concentration GaGdN and Si-doped GaGdN grown at low temperatures, Applied Physics Letters 92(6), 062505, 2008

[24] F.-Y. Lo, A. Melnikov, D. Reuter, A. D. Wieck, V. Ney, T. Kammermeier, A. Ney, J. Schörmann, S. Potthast, D. J. As, K. Lischka, Magnetic and structural properties of Gd-implanted zinc-blende GaN, Applied Physics Letters $\mathbf{9 0}(26), 262505,2007$

[25] A. Bedoya-Pinto, J. Malindretos, M. Roever, D. D. Mai, A. Rizzi, Variable range hopping transport in ferromagnetic GaGdN epitaxial layers, Physical Review B 80(19), 195208, 2009

[26] S. Dhar, T. Kammermeier, A. Ney, L. Perez, K. H. Ploog, A. Melnikov, A. D. Wieck, Ferromagnetism and colossal magnetic moment in Gd-focused ionbeam-implanted GaN, Applied Physics Letters 89(6), 062503, 2006

[27] M. A. Khaderbad, S. Dhar, L. Perez, K. H. Ploog, A. Melnikov, A. D. Wieck, Effect of annealing on the magnetic properties of Gd focused ion beam implanted GaN, Applied Physics Letters 91(7), 072514, 2007

[28] F.-Y. Lo, A. Melnikov, D. Reuter, Y. Cordier, A. D. Wieck, Magnetotransport in Gd-implanted wurtzite GaN/ $\mathrm{Al}_{\mathrm{x}} \mathrm{Ga}_{1-\mathrm{x}} \mathrm{N}$ high electron mobility transistor structures, Applied Physics Letters 92(11), 112111, 2008

[29] S. Y. Han, J. Hite, G. T. Thaler, R. M. Frazier, C. R. Abernathy, S. J. Pearton, H. K. Choi, W. O. Lee, Y. D. Park, J. M. Zavada, R. Gwilliam, Effect of Gd implantation on the structural and magnetic properties of GaN and AlN, Applied Physics Letters 88(4), 042102, 2006

[30] A. Ney, T. Kammermeier, V. Ney, S. Ye, K. Ollefs, E. Manuel, S. Dhar, K. H. Ploog, E. Arenholz, F. Wilhelm, A. Rogalev, Element specific magnetic properties of Gd-doped GaN: Very small polarization of Ga and paramagne- 
tism of Gd, Physical Review B (Condensed Matter and Materials Physics) 77(23), 233308, 2008

[31] J. K. Hite, R. M. Frazier, R. Davies, G. T. Thaler, C. R. Abernathy, S. J. Pearton, J. M. Zavada, Effect of growth conditions on the magnetic characteristics of GaGdN, Applied Physics Letters 89(9), 092119, 2006

[32] J. S. Blakemore, Semiconducting and other major properties of gallium arsenide, Journal of Applied Physics 53(10), R123, 1982

[33] M. A. Reshchikov, H. Morkoç, Luminescence properties of defects in GaN, Journal of Applied Physics 97(6), 061301, 2005

[34] A. Svane, N. E. Christensen, L. Petit, Z. Szotek, W. M. Temmerman, Electronic structure of rare-earth impurities in GaAs and GaN, Physical Review B (Condensed Matter and Materials Physics) 74(16), 165204, 2006

[35] Y. Gohda, A. Oshiyama, Intrinsic ferromagnetism due to cation vacancies in Gd-doped GaN: First-principles calculations, Phys. Rev. B 78(16), 161201, 2008

[36] G. M. Dalpian, S.-H. Wei, Electron-induced stabilization of ferromagnetism in $\mathrm{Ga}_{1-\mathrm{x}} \mathrm{Gd}_{\mathrm{x}} \mathrm{N}$, Phys. Rev. B 72(11), 115201, 2005

[37] L. Liu, P. Y. Yu, Z. Ma, S. S. Mao, Ferromagnetism in GaN:Gd: A Density Functional Theory Study, Physical Review Letters 100(12), 127203, 2008

[38] C. G. Van de Walle, J. Neugebauer, First-principles calculations for defects and impurities: Applications to III-nitrides, Journal of Applied Physics 95(8), 3851, 2004

[39] J. Neugebauer, C. G. Van de Walle, Gallium vacancies and the yellow luminescence in GaN, Applied Physics Letters 69(4), 503, 1996

[40] A. F. Wright, Substitutional and interstitial oxygen in wurtzite GaN, Journal of Applied Physics 98(10), 103531, 2005

[41] J. Neugebauer, C. G. V. de Walle, Chemical trends for acceptor impurities in GaN, Journal of Applied Physics 85(5), 3003, 1999

[42] J. Neugebauer, C. G. Van de Walle, Role of hydrogen in doping of GaN, Applied Physics Letters 68(13), 1829, 1996

[43] J. Oila, V. Ranki, J. Kivioja, K. Saarinen, P. Hautojärvi, J. Likonen, J. M. Baranowski, K. Pakula, T. Suski, M. Leszczynski, I. Grzegory, 
Influence of dopants and substrate material on the formation of Ga vacancies in epitaxial GaN layers, Phys. Rev. B 63(4), 045205, 2001

[44] K. Saarinen, T. Laine, S. Kuisma, J. Nissilä, P. Hautojärvi, L. Dobrzynski, J. M. Baranowski, K. Pakula, R. Stepniewski, M. Wojdak, A. Wysmolek, T. Suski, M. Leszczynski, I. Grzegory, S. Porowski, Observation of Native Ga Vacancies in GaN by Positron Annihilation, Phys. Rev. Lett. 79(16), 3030, 1997

[45] A. F. Wright, Substitutional and interstitial carbon in wurtzite GaN, Journal of Applied Physics 92(5), 2575, 2002

[46] P. Dev, Y. Xue, P. Zhang, Defect-Induced Intrinsic Magnetism in Wide-Gap III Nitrides, Physical Review Letters 100(11), 117204, 2008

[47] P. Dev, P. Zhang, Unconventional magnetism in semiconductors: Role of localized acceptor states, Phys. Rev. B 81(8), 085207, 2010

[48] A. Bedoya-Pinto, Structural, magnetic and electrical transport properties of GaNbased magnetic semiconductors and hybrid structures, Dissertation, 2010

[49] C. Mitra, W. R. L. Lambrecht, Interstitial-nitrogen- and oxygen-induced magnetism in Gd-doped GaN, Physical Review B (Condensed Matter and Materials Physics) 80(8), 081202, 2009

[50] P. Paskov, T. Paskova, P. Holtz, B. Monemar, Internal Structure of Free Excitons in GaN, phys. stat. sol. (b) 228(2), 467, 2001

[51] J. Creighton, D. Koleske, C. Mitchell, Emissivity-correcting near-UV pyrometry for group-III nitride OMVPE, Journal of Crystal Growth 287(2), 572, 2006

[52] J. Creighton, W. Breiland, D. Koleske, G. Thaler, M. Crawford, Emissivity-correcting mid-infrared pyrometry for group-III nitride MOCVD temperature measurement and control, Journal of Crystal Growth 310(6), 1062 , 2008

[53] K. G. Tschersich, Intensity of a source of atomic hydrogen based on a hot capillary, Journal of Applied Physics 87(5), 2565, 2000

[54] E. Franke, Entwicklung einer optischen Prozesskontrolleinheit für Stickstoffplasmaquellen, Bachelor thesis, 2009

[55] H. Hollermann, Der Einfluss verschiedener Stickstoffspezies auf das GaNWachstum mittels Molekularstrahlepitaxie, Bachelor Thesis, 2010 
[56] S. Dhar, O. Brandt, M. Ramsteiner, V. F. Sapega, K. H. Ploog, GaN:Gd: A superdilute ferromagnetic semiconductor with a Curie temperature above $300 \mathrm{~K}$, arXiv.org:cond-mat/0412564, 2004

[57] M. Sawicki, private Mitteilungen, 2010

[58] D. B. McWhan, Effect of Pressure on the Curie Temperature and Volume of GdN, The Journal of Chemical Physics 44(9), 3528, 1966

[59] D. X. Li, Y. Haga, H. Shida, T. Suzuki, Magnetic properties of ferromagnetic GdN, Physica B: Condensed Matter 199-200, 631, 1994

[60] O. Hitzemann, Untersuchungen an verdünnten magnetischen Halbleitern als Materialien für die Spintronik, Diplomarbeit, 2010

[61] K. Saarinen, in M. O. Manesreh (Editor), III-V Nitride Semiconductors: Electrical, Structural and Defects Properties, p. 109, Elsevier, Amsterdam, 2000

[62] C. Rauch, private Mitteilungen, 2010

[63] J. Zenneck, Optische Eigenschaften von verdünnten magnetischen Halbleitern auf GaN-Basis, Ph.D. thesis, Georg-August-Universität Göttingen, 2007

[64] A. Wysmołek, M. Kamińska, A. Twardowski, M. Potemski, M. Boćkowski, I. Grzegory, Magneto-Luminescence of gadolinium doped gallium nitride, International Journal of Modern Physics B 23(12 \& 13), 2994, 2009

[65] S. W. Choi, S. Emura, S. Kimura, M. S. Kim, Y. K. Zhou, N. Teraguchi, A. Suzuki, A. Yanase, H. Asahi, Emission spectra from AlN and GaN doped with rare earth elements, Journal of Alloys and Compounds 408-412, 717, 2006

[66] A. Shikanai, T. Azuhata, T. Sota, S. Chichibu, A. Kuramata, K. Horino, S. Nakamura, Biaxial strain dependence of exciton resonance energies in wurtzite GaN, Journal of Applied Physics 81(1), 417, 1997

[67] J. A. Freitas, W. J. Moore, B. V. Shanabrook, G. C. B. Braga, S. K. Lee, S. S. Park, J. Y. Han, Donor-related recombination processes in hydridevapor-phase epitaxial GaN, Phys. Rev. B 66(23), 233311, 2002

[68] V. Y. Davydov, N. S. Averkiev, I. N. Goncharuk, D. K. Nelson, I. P. Nikitina, A. S. Polkovnikov, A. N. Smirnov, M. A. Jacobson, O. K. Semchinova, Raman and photoluminescence studies of biaxial strain in GaN epitaxial layers grown on 6H-SiC, Journal of Applied Physics 82(10), 5097, 1997 
[69] R. Y. Korotkov, F. Niu, J. M. Gregie, B. W. Wessels, Investigation of the defect structure of GaN heavily doped with oxygen, Physica B: Condensed Matter 308-310, 26, 2001

[70] Z. Lipińska, M. Pawłowski, H. Zołnierowicz, A. Wysmołek, M. Palczewska, M. Kamińska, A. Twardowski, M. Boćkowski, I. Grezegory, Photoluminescence and Electron Paramagnetic Resonance Studies of Bulk GaN doped with Gadolinium, Acta Physica Polonica A 110, 243, 2006

[71] Hertz, Ueber die Verdunstung der Flüssigkeiten, insbesondere des Quecksilbers, im luftleeren Raume, Annalen der Physik 253, 177, 1882

[72] M. A. Herman, H. Sitter, Molecular Beam Epitaxy, Springer, 1996

[73] W. Martienssen, H. Warlimont (Editors), Springer Handbook of Condensed Matter and Materials Data, Springer, 2005 


\section{Danksagungen}

Prof. Dr. Angela Rizzi

Für die Möglichkeit im spannenden Gebiet der Verdünnten Magnetischen Halbleiter meine Dissertation zu schreiben, das Vertrauen in meine Arbeit und nicht zuletzt für die immer herzliche Atmosphäre in der gesamten Arbeitsgruppe.

\section{Prof. Dr. Axel Hoffmann}

Für die schnelle und unkomplizierte Übernahme des Korreferates.

\section{Dr. Jörg Malindretos}

Das Lesen der Arbeit, die vielen Diskussionen über die Physik, das Aufräumen im Labor ;) und die geduldigen Erklärungen bei jeglichen Fragen. Außerdem als gutem Freund neben der Uni, das Kochen und Basteln, die Bewerbungsfotos und den Whisky.

\section{Christian und Borris}

Für die mehr als angenehme Bürogesellschaft. (Dieser Punkt ist mit Absicht so kurz gehalten. Das ist nun mal so bei Norddeutschen...)

\section{Physikalisches Institut}

Für die angenehme Arbeitsatmosphäre und die schönen Zeiten mit allen Institutskollegen. Insbesondere werden mir dort auch immer das Institutsseminar in Riezlern in Erinnerung bleiben, auf dem ich das Skifahren lernte.

\section{Allen Freunden}

... die ich hier nicht alle namentlich nenne, da sie sich in den folgenden Worten selbst wiederfinden. Feiern, Kochen, Klettern, Windsurfen, Festivals, Reisen, Schrauben, Lachen und Weinen, Ablenkung von der Uni, Unterstützung bei der Arbeit und einfach dafür, daß es euch gibt!

\section{Meiner Mutter}

Für Vertrauen, Geduld, die tolle Reise nach China. 


\section{Lebenslauf}

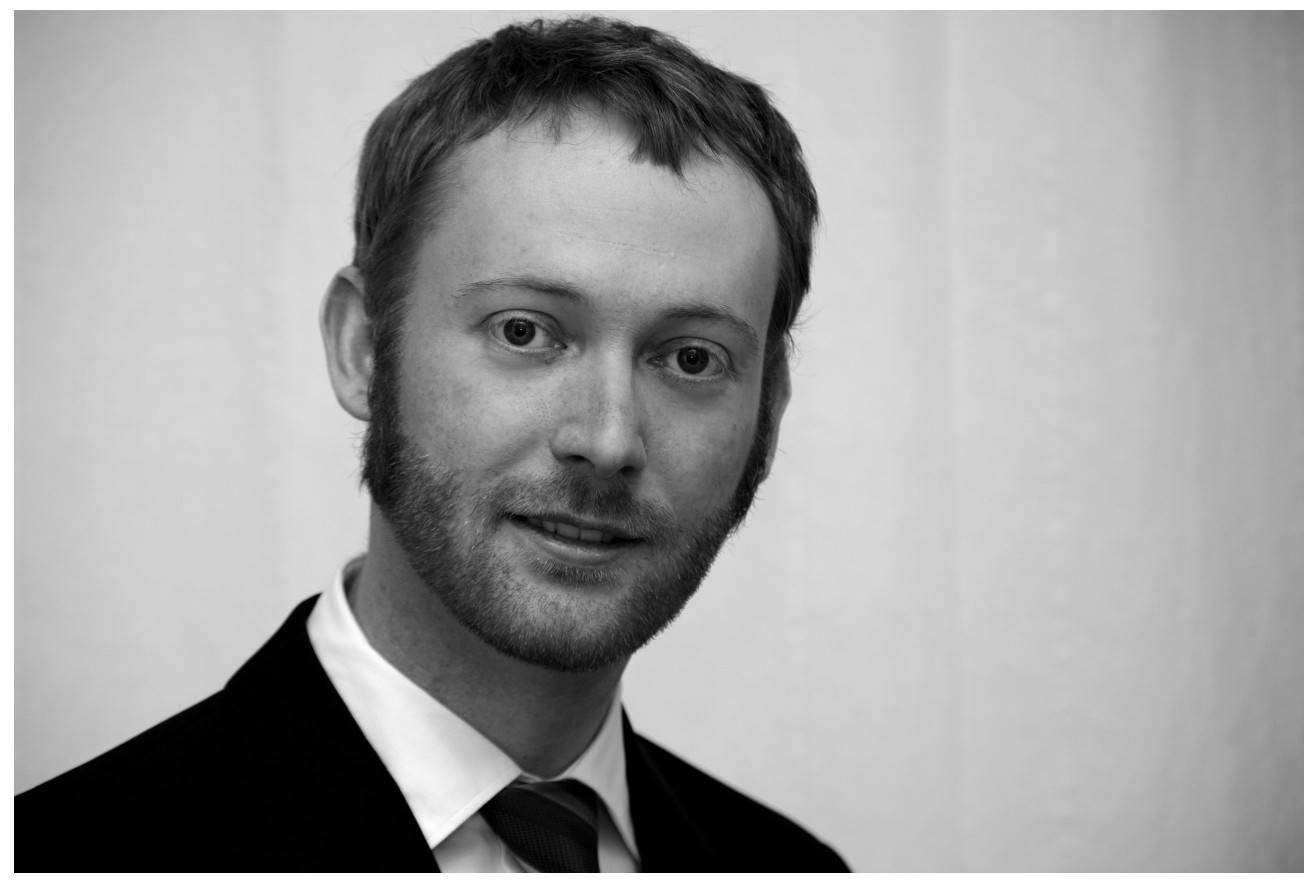

\section{Persönliche Daten}

$\begin{array}{ll}\text { Vor- und Zuname } & \begin{array}{l}\text { Martin Röver } \\ \text { Adresse }\end{array} \\ & \begin{array}{l}\text { Albrecht-von-Haller-Str. 2 } \\ \text { Geburtsdatum }\end{array} \\ \text { Föttingen } \\ \text { Familienstand } & \text { ledig } \\ \text { Staatsangehörigkeit } & \text { deutsch }\end{array}$




\section{Schul- und Hochschulausbildung}

08.2005 - 10.2010 Promotion, IV. Physikalisches Institut, Halbleiterphysik, GeorgAugust-Universität, Göttingen, Abschlußnote: magna cum laude. Titel der Dissertation: „GaN:Gd - Ein verdünnter magnetischer Halbleiter?"

10.2000 - 07.2005 Diplomstudium der Physik mit Schwerpunkt Festkörperphysik an der Georg-August-Universität Göttingen.

Titel der Diplomarbeit: „GaMnN - MBE auf Si(111), Einfluß von Wachstumstemperatur und III-Mn-V Verhältnis auf die strukturellen Eigenschaften und den Manganeinbau“"

08.1993 - $06.2000 \quad$ Ubbo-Emmius-Gymnasium Leer, Abitur

\section{Praktische Erfahrung}

seit 08.2005

Wissenschaftlicher Mitarbeiter, IV. Physikalisches Institut, Halbleiterphysik, Georg-August-Universität Göttingen:

Verantwortung über das Molekularstrahlepitaxie-Labor. Herstellung und Optimierung von III-N-Halbleitern. Strukturelle, magnetische und optische Charakterisierung von III-N-Halbleitern. Betreuung von Diplomarbeiten und Lehrtätigkeit an der Fakultät für Physik.

Angewandte Methoden: molecular beam epitaxy (MBE), superconducting quantum interference device (SQUID), photoluminescence (PL), x-ray photon spectroscopy (XPS), x-ray diffraction (XRD), reflection high energy electron diffraction (RHEED), auger electron spectroscopy (AES), energy dispersive x-ray (EDX), atomic force microscopy (AFM), scanning electron microscopy (SEM).

Fundiertes Wissen über UHV Technik und sehr sicherer Umgang mit dieser.

08.2005 - 10.2008 Assistent im Seminar „Physik für Mediziner und Zahnmediziner“ Vermittlung der Grundlagen der Physik im Rahmen von Blockveranstalltungen für Medizinstudenten.

03.2004 - 08.2005 Tutor im Praktikum „Physik für Mediziner und Zahnmediziner“ Versuchsbegleitung im Rahmen von Blockveranstalltungen für Medizinstudenten. 